\title{
THE EVERYDAY HEALTH GEOGRAPHIES OF BHUTANESE AND NEPALESE MIGRANTS IN OTTAWA: HEALTH, WELLBEING AND THERAPEUTIC NETWORKS
}

\section{BY}

\section{ASLI MAHDI}

A thesis submitted to the Faculty of Graduate and Postdoctoral Affairs in partial fulfillment of the requirements for the degree of Master of Arts

Department of Geography and Environmental Studies Carleton University

Ottawa, Ontario

(C) Copyright

September 2015, Asli Mahdi 


\begin{abstract}
The health geographies of recent migrants have been little explored in geography. As well, there has been little effort to consider what "health" and "wellbeing" mean to people, especially racialized peoples, in the literature. Insights from health geography and postcolonial theory in this research highlight how these concepts can be best understood through a lens on everyday life. My research examined perceptions of health and wellbeing through semi-structured interviews with several Nepalese and Bhutanese migrants in Ottawa. The research revealed that both health and wellbeing can be read through daily routines, and five themes were identified: i) definitions of health and wellbeing; ii) ideas of health related to food and cooking; iii) health and wellbeing associated with walking; iv) health, employment, and income independence; and v) the role(s) of the neighbouring community in health maintenance. Overall, I suggest that these narratives of health, which capture pre-migration memories in post-migration contexts, should be seen by researchers as forms of health knowledges and lived experiences that reveal particular notions and places associated with health and wellbeing. This study also critically examined the research process, highlighting the several roadblocks and issues with recruitment-related challenges, addressing issues of researcher self-esteem, as well as reflexivity in qualitative research. Here, I propose that health geographers need to also pay greater attention to their own lived health geographies while both "on" and "off" the field, to give a more holistic picture of their experiences as a researcher. Overall, this study strongly suggests the value of further research on the everyday health realities and geographies of different immigrants and refugee groups in Canada, to highlight how health and wellbeing can be differently conceptualized and lived.
\end{abstract}




\section{Acknowledgments}

I would like to thank my supervisors, Dr. Fran Klodawsky and Dr. Paul Mkandawire for all their support, guidance and patience for the last two years on this project, especially for the advice you both gave me in thinking about reflexivity, and I hope this is evident in this thesis.

For Carleton's Anthropology department that sowed the seeds for this project years prior. For Kristin Bright, thank you for introducing me to medical anthropology, had I not taken your course, this research may have looked very different.

I would also like to acknowledge the hard work of Abha Saytal and Siffan Rahman from the Ottawa Newcomer Clinic, for their interest in this research, and getting it approved. For Abha specifically, I am thankful for all your help in recruitment and interpretation. The time I spent with participants was a very enjoyable experience and integral to this project. Thank you both.

For those in the Geography department, both students and staff, thank you for your assistance and support.

For close friends who have assisted me through open ears and encouragement, you are appreciated.

Most importantly, I am indebted to my mother and siblings for their support and unconditional love for the last 24 years. This would not have happened without you all. 


\section{Table of Contents}

\section{THE EVERYDAY HEALTH GEOGRAPHIES OF BHUTANESE AND NEPALESE MIGRANTS IN OTTAWA: HEALTH, WELLBEING AND THERAPEUTIC NETWORKS}

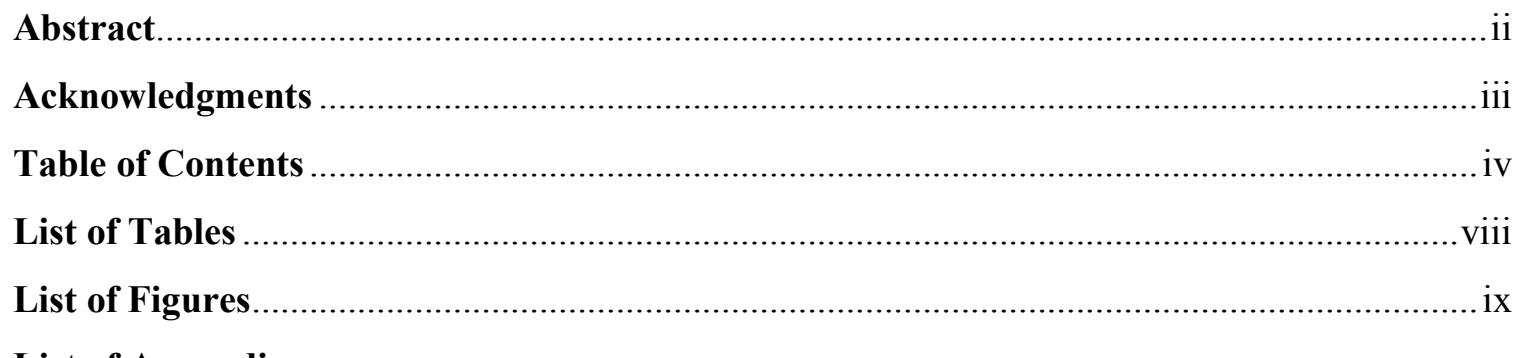

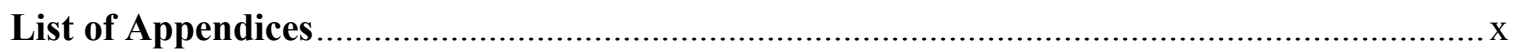

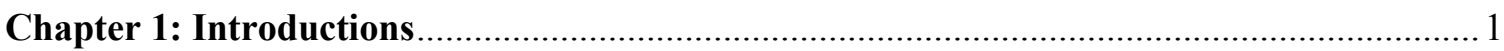

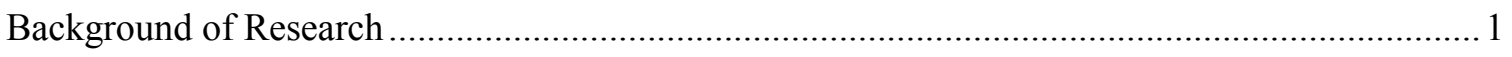

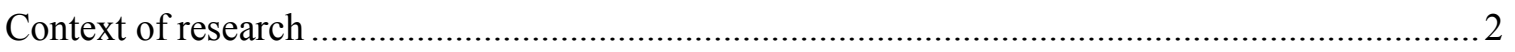

Immigration Acts and Nepalese and Bhutanese migration ................................................. 2

Unpacking health and place: the Social Determinants of Health.............................................. 7

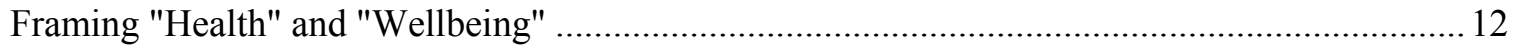

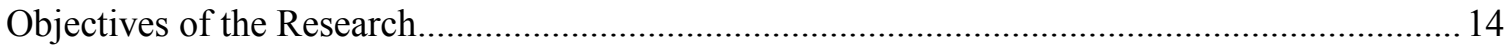

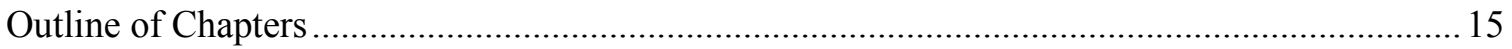

Chapter 2: Theoretical framework and review of the literature ......................................... 19

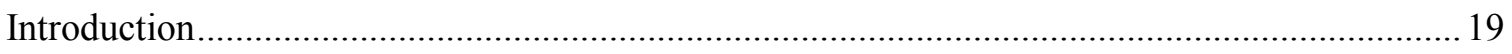

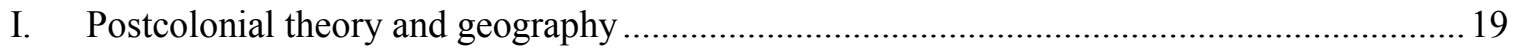

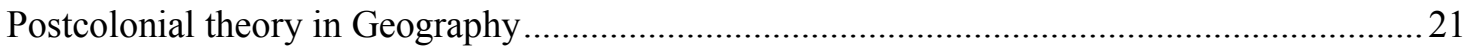

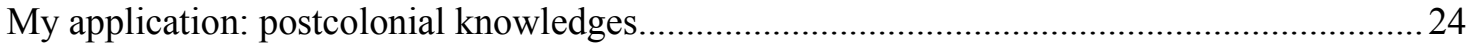

II. Place in medical and health geography: discussions and applications................................22

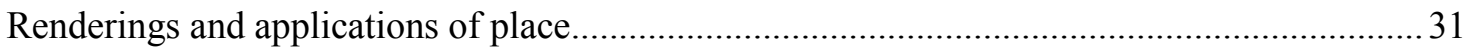

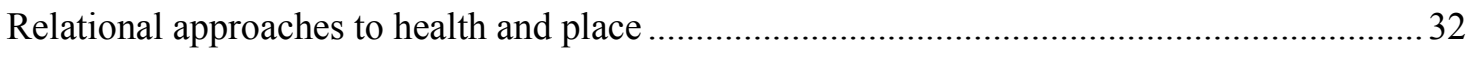

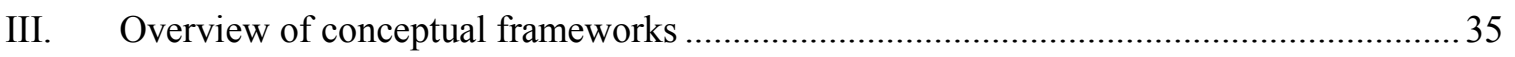




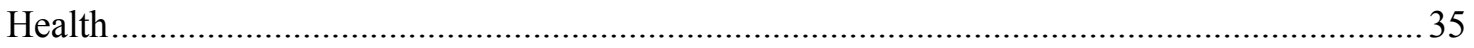

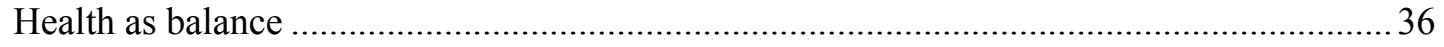

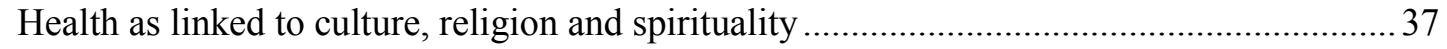

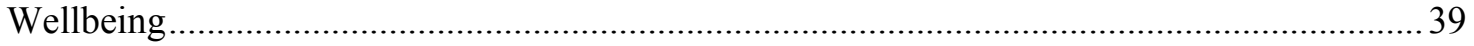

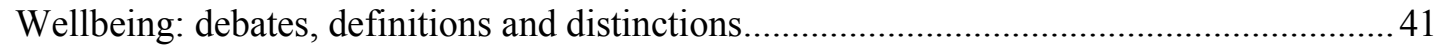

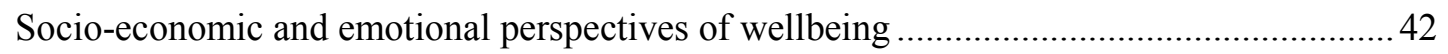

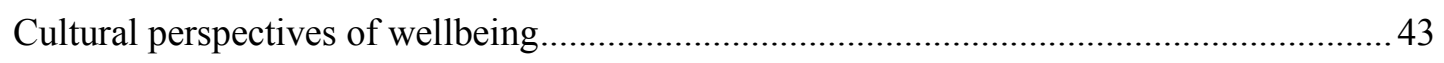

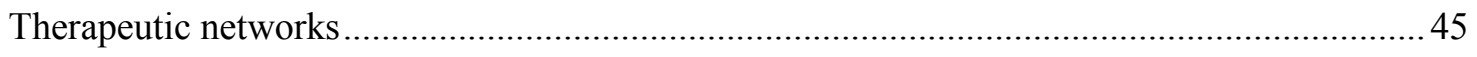

IV. Health and place: everyday health geographies .......................................................... 47

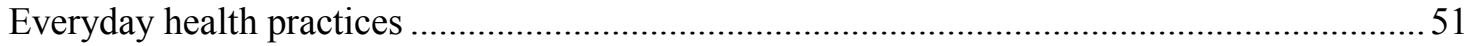

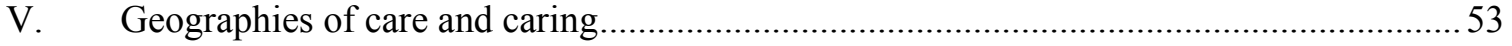

Care as practice

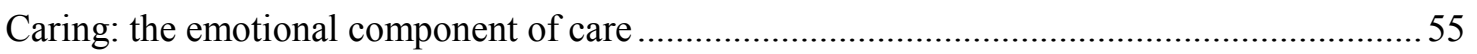

VI. Everyday geographies of immigrant and refugee health..............................................5

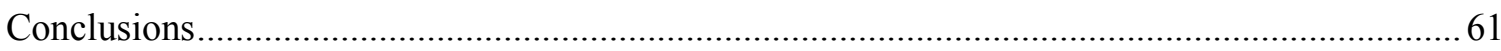

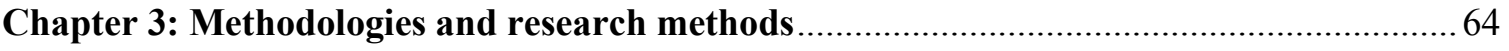

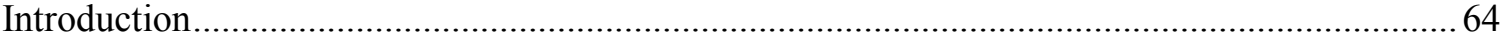

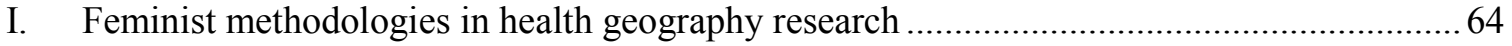

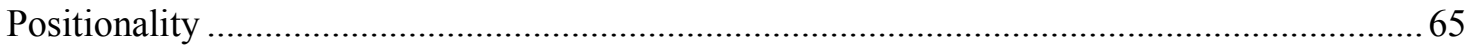

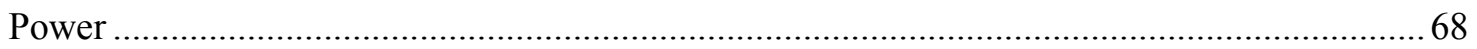

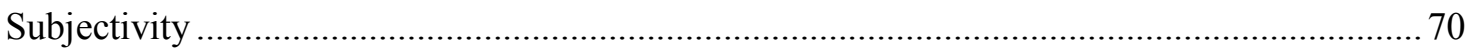

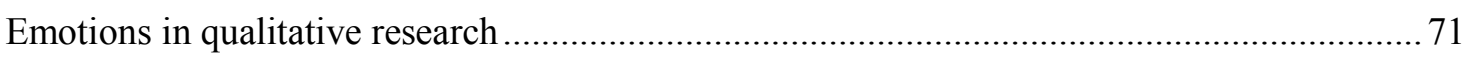

II. Reflexivity: rejoice, rejection and redemption of research ............................................. 73

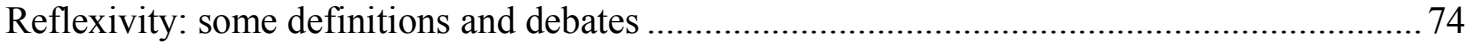

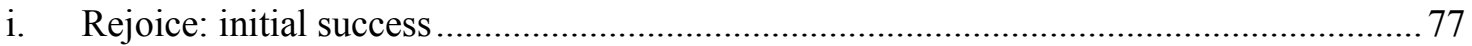

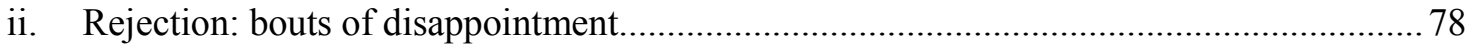

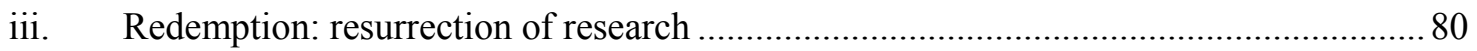

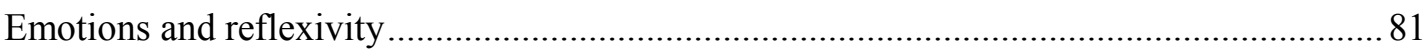

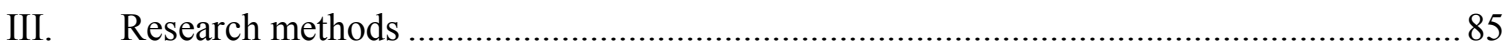

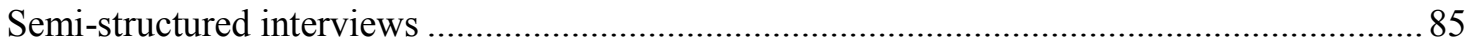

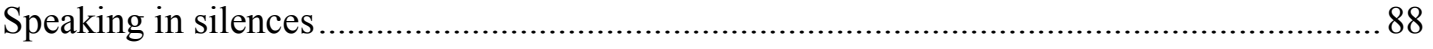

Interpreters in cross-cultural interviews: power relations and ethics ................................... 89 
Micro-geographies: the role of place in semi-structured interviews................................. 92

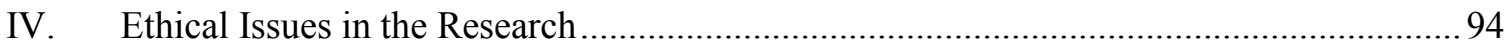

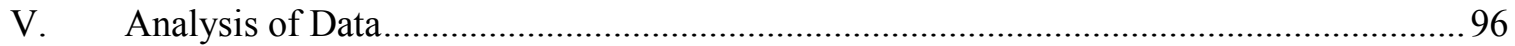

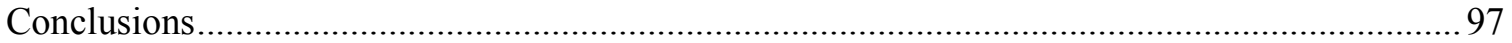

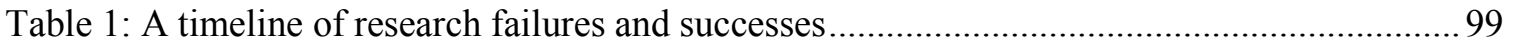

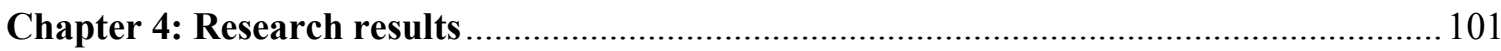

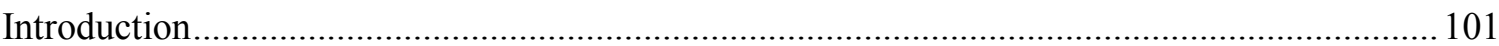

Refugee or Immigrant: the contextual role of immigration status ....................................... 102

Table 2: Participant table, organized by age, gender, nationality and immigrant status ...... 102

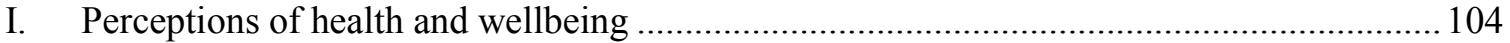

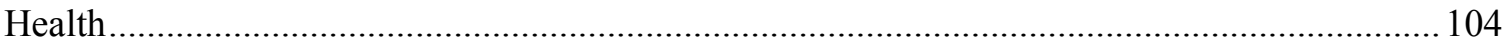

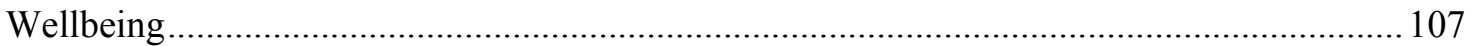

II. Steps to wellbeing: walking and mobile geographies of health ......................................... 109

III. Consuming health: the role of food, cooking and cleanliness ....................................... 112

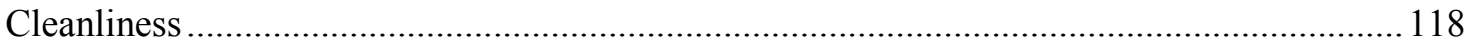

IV. Working towards health: employment and income and its effects on health and

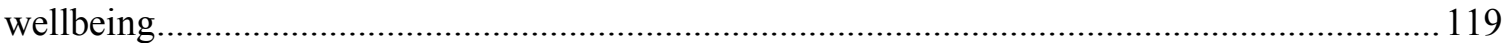

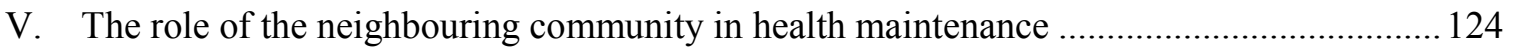

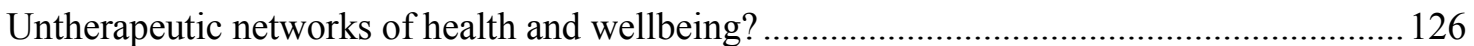

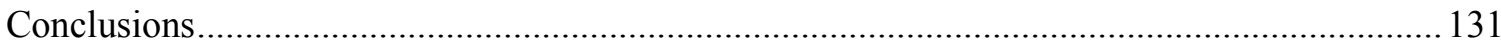

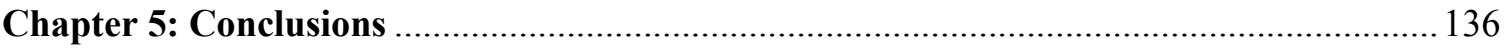

I. A geography of health and wellbeing: overview of research ......................................... 136

The 'place' of health, wellbeing and therapeutic networks in the literature ............................. 137

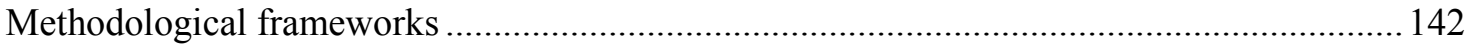

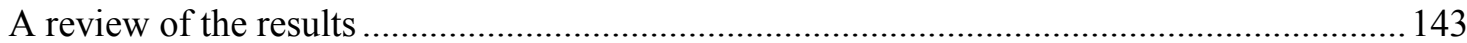

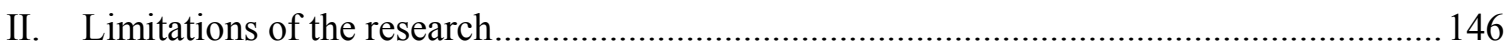

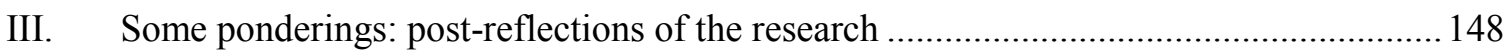

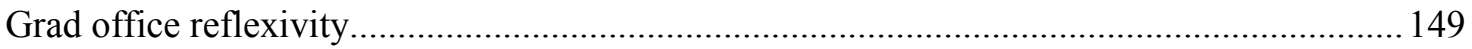

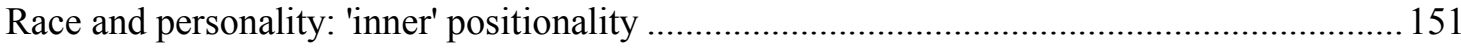

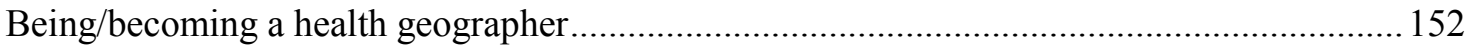

IV. Suggestions for future research in health geography ................................................... 154 


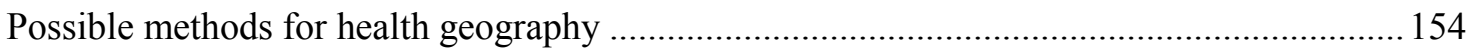

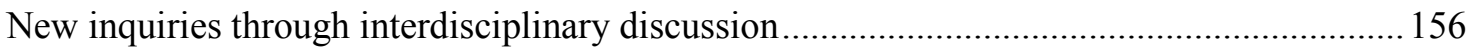

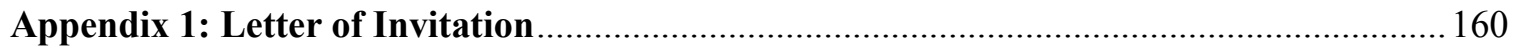

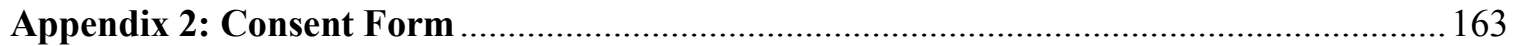

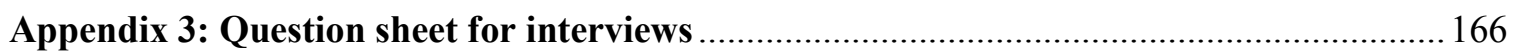

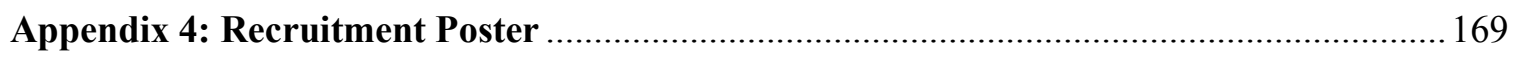

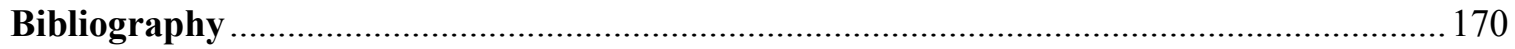




\section{List of Tables}

Table 1: A timeline of research failures and success

pages $99-100$

Table 2: Participant table, organized by age, gender, nationality and immigration status

page 102 


\section{List of Figures}

Figure 1: The Social Determinants of Health

page 9

ix 


\section{List of Appendices}

Appendix 1: Letter of Invitation

Appendix 2: Consent Form

Appendix 3: Question Sheet for interviews

Appendix 4: Recruitment Poster pages $160-162$

pages $163-165$

pages $166-168$

page 169 


\section{Chapter 1: Introductions}

\section{Background of Research}

My decision to pursue this research came from my undergraduate background in anthropology. In my second year, I enrolled in a medical anthropology course, which exposed to me ways in which health could be explored a cultural lens. The following year, I took a cultural geography course, which introduced to me avenues in which geography could be used as a lens to understand practices, like cooking, in relation to colonialism, to globalization. By blending medical anthropology and cultural geography, I had hoped I would be able to research how health and wellbeing could be understood through the lenses of culture and place. Literature in an emerging health geography helped me make these theoretical links more salient. I came across the works of health geographer Isabel Dyck, well-known for her work on women's health geographies (1995a, 1995b, 2006). One of her first pieces looked at the micro-geographies of women with multiple sclerosis (Dyck, 1995b). One of the key points she made in this work was the importance in recognizing how places are constructed from interactions between the body, space and practice. In particular, two of her works were very much related to what I wanted to research. The first, published in 2006, examined the daily health practices of Punjabi Sikh immigrant women in Vancouver. In this work, she found that health took place in women's daily lives, woven in food practices, traditional remedies or work, showing that health is also cultural. This work was later adapted into another piece with medical anthropologist, Parin Dossa (2007). This work looked at the health practices of Punjabi Sikh and Muslim Afghan women, contrasting their experiences, as immigrants 
and refugees, and how they conceived of health. Some women pointed to religious practices, such as prayer, while others referred to food and accessing local health services. This thesis examines the everyday geographies of Nepalese and Bhutanese migrants in Ottawa, Canada. The research particularly focuses on how notions of health and wellbeing can be illustrated through attention to the daily routines people perform. The purpose of this research is to highlight how health and wellbeing can be revealed in different aspects of one's life, particularly through the use of semi-structured interviews. All the participants for this study were recruited through community health workers at the Newcomer Clinic in Ottawa. The Newcomer Clinic, formally known as the Wellness Centre is located on 219 Argyle Avenue in Ottawa and is affiliated with Somerset Community Health Centre. Its role is to provide medical screening for new clients, as well as serve their urgent medical needs (SWCHC, 2012). The Clinic, like community health centres, also serves a health promotion function, and Abha, one of the two major gatekeepers for this project, served as a community health navigator for the Bhutanese population in Ottawa. Before discussing this research further, it is important to situate the context of this research.

\section{Context of research}

\section{Immigration Acts and Nepalese and Bhutanese migration}

An important piece of legislature to note in immigrant and refugee research is The Immigration Act, 1962. The Immigration Act in 1962 was paramount because immigrants and refugees were admitted into the country not based on their race or national origin but through a points system. In 1967, further changes were made when the Points System 
was created, where "immigrants were assigned points on a scale of 0 to 10 (or 15) based on the qualities listed directly above. If they reached a certain level of points in total, they were allowed into the country, and there were no quotas or restrictions placed on the number of people who could immigrate" (Canadiana, 2005). This Points System, however, did not take into account the situation of refugee groups, many likely having their education delayed due to war or political turmoil in their countries of origin. The 1967 reformulation of the Immigration Act made strides to define more closely the different types of immigrants, in this case, four: refugees, family immigrants, assisted relatives, and independent or economic immigrants (Canadiana, 2005). This specification of different immigrants was very important because it meant that "other classes did not have to take part in this test so long as they passed basic criminal, security and health checks" (Canadiana, 2005). This version of the Immigration Act is very significant for South Asian immigration to Canada, as South Asians belonged to different immigrant classes.

Bhutanese and Nepalese migration routes to Canada is what is of interest to this research. Nepalese immigration to Canada occurred in the last five or six years to Canada, though with many arriving in the late 1980s and 1990s as well. However, what distinguishes them from the Bhutanese is their immigration status. The Nepalese primarily arrived to Canada in the skilled immigrant class. In addition, many have lived in other countries, such as the United States, the UK, India, and Hong Kong before arriving to Canada (Multicultural Canada, n.d.). As mentioned, many of the Nepalese came to Canada fairly educated, with many in high paying jobs in the private sector. What also sets them apart from the Bhutanese is the large community organizations 
catering to them, including the Nepalese Association in Canada from 1993 in Ontario, and the Canada-Nepal Friendship Association, which began in 1989 in Ottawa (Multicultural Canada, n.d.). What is also notable is their contribution and influence on Nepalese politics back in Nepal, as well as other transnational connections.

According to Citizenship and Immigration Canada (2007), the reason for the emigration of Bhutanese people was a result of the Bhutanese government displacing the Lhotshampa in the country. Rizal (2004) explains that the 1980s in Bhutan was a period of major changes in the country. Requirements for citizenship was one route which excluded Lhotshampa. Because citizenship is based on ethnicity, the Lhotshampas, not being a Drukpa or speaking Dzongkha, the national language, are required to obtain citizenship by naturalization. As a result, "the Lhotshampa were asked to show 30-year old land-tax receipts as proof of their citizenship under the 1958 Act. However, since payment of land-tax was mandated across the country only in 1964, this requirement was particularly difficult for the Lhotshampa to fulfill" (p. 159). These contradictory requirements was one way the monarchical government sought to deny minority groups in obtaining citizenship,

Furthermore, a cultural policy, called driglam namzha, was imposed which requires the Lhotshampa and other minority groups to wear Buddhist national dress and take training in Drukpa traditional etiquette (p. 156). From this, the teaching of Nepalese, the language spoken by the Lhotshampa was then prohibited in 1989. One of the major policies which illustrated political changes in Bhutan was the 'One Nation, One People' policy. Rizal (pp. 160-161) explains that the

'One Nation, One People' platform, focuses on what the government claims is the need for a distinct 'national identity' - an ethnically exclusive Kargupa Buddhist 
identity. This policy seeks to explicitly purge Bhutanese society of its diversity by forcibly imposing Drukpa values, lores, symbols and traditions in a multi-ethnic and multicultural society. This policy intends to make a nation not just of 'One People' but more diacritically of 'One Drukpa People'.

This attention to the promotion of Drukpa cultural values was a part of the Drukpa revivalism movement in the 1980 s, a way to erase the multicultural elements of Bhutanese society. Rizal notes that this cultural movement even had geographical effects, through the changing of Lhotshampa Nepali place-names to Drukpa-sounding names (pp. 163, 164). As well, religion also played a major role here. Rizal explains that because the Drukpa people are primarily Buddhist, while the Lhotshampa and other minority groups are predominately Hindu, religion became an arena of major friction, as it was assumed that these religious differences could negatively affect the so-called unity of the country (p. 163). Much like religion, linguistic difference also placed the Lhotshampa in suspicion, as the government at times accused the Nepali-speaking Lhotshampa of contributing to a 'Greater Nepal' movement, undermining sovereignty. Instead, Rizal (p. 164) notes that the Lhotshampa are more interested in maintaining sovereignty.

Alongside these assimilation policies, the monarchical government also posed other laws, such as the no Objection Certificate. This certificate was required from the Lhotshampa to obtain basic rights as citizens, such as "children's admission into school, promotion in the civil service, a passport, a state scholarship, eligibility to take civilservice examinations and even apply for jobs in the government service" (p. 160). However, in the face of Lhotshampa peaceful protests in the fall of 1990, these certificates could then be revoked. Anyone who participated, had family who participated, or even sympathized with the movement was at the risk of getting their certificate annulled (p. 160). This measure denied the right for many Lhotshampa 
children from attending school.

These many state measures that promoted inequality in the country resulted in over 100000 ethnic Nepalese from Bhutan moving into refugee camps in Nepal between 1988 and 1993. Although bilateral talks have occurred between Bhutan and Nepal, Rizal reminds us that while these are going on, the lands of people forced to leave are now being resettled by landless groups from northern Bhutan. Rizal insists that this move was not one to "'settle the landless, who have been landless since long before 1997. This program is permanently to prevent the repatriation of refugees and force them to assimilate in Nepal" (p. 168). Life in these camps is mixed. The Kenan Institute of Duke University (2013) describe the situations in these refugee camps:

Life in the camps can be very difficult. Hard work is mixed with extreme idleness. Refugees must build and repair their own huts and transport heavy loads on their backs. Skilled workers can spend hours sewing or practicing metalwork or carpentry. The camps offer little in the way of recreation, and there are no opportunities for legal paid employment in Nepal. For a community that strongly values an active lifestyle and hard work, there's little chance to put those values to productive use.

Here, the inability to utilize employment skills and the labour required to maintain one's home in the camps seems to prevent the Bhutanese from participating in broader Nepalese society. From living in the camps, it is also suggested that relationships with broader Nepalese society is also mixed, with the camps blamed for deforestation. However, positive relationships between the Nepalese has also been sparked, in the form of friendships and marriages (Duke University, 2013).

Since 2005, the numbers of Bhutanese refugees have grown large. Citizenship and Immigration Canada estimates that "as of March 31, 2013, more than 5760 Bhutanese refugees have arrived in Canada", with communities spanning from the Maritime 
provinces to British Columbia (Duke University, 2013). In a Globe and Mail article (Armstrong, 2009), the challenges faced by Bhutanese refugees in Vancouver were focused on:

Unlike most immigrants and refugees, the government-sponsored Bhutanese don't have the safety net of an already established community to ease their culture shock and offer support. That lack of a support group has prompted questions about how they will assimilate in their new country. Their backgrounds don't lend themselves to easy adaptation. They have spent 17 years in refugee camps and most of the adults have never held full-time jobs.

Here, barriers to adjusting to Canadian society appears to be related to a lack of established social networks and capital to draw on for support. However, what is unfortunate is the little research investigating these resettlement issues more closely. This is where my research begins, with attention to how notions of health and wellbeing can be understood geographically, in the daily lives of Bhutanese and Nepalese people.

\section{Unpacking health and place: the Social Determinants of Health}

The relationships between health, wellbeing and place is of particular interest in this research. The best way to expose these links between health and place, this research suggests, is to use a relational lens, which recognizes that as people's relationships to place change and ideas of health shift, so do the links between health and place (cf. Cummins et al., 2007). This relational framework towards health is also evident in Canadian health policy. For example, the Social Determinants of Health in Canada recognizes that a person's health can be influenced by one's gender, age, place of residence, and one's social and work environments. however, what is lacking in Canadian health policy is the consideration that for immigrants and refugees, there may be different health determinants at play. The Social Determinants of Health model is important to 
mention in this research because it illustrates how one's health can be affected by many different determinants. Considering facets of one's identity, like gender, while also taking into account the everyday geographies, like employment, as well as access to education, illustrate the importance of health in many aspects of one's life. Raphael $(2009$, p. 2) defines social determinants as

the economic and social conditions that shape the health of individuals, communities and jurisdictions as a whole. Social determinants of health are the primary determinants of whether individuals stay healthy or become ill. Social determinants of health also determine the extent to which a person possesses the physical, social, and personal resources to identify and achieve personal aspirations, satisfy needs, and cope with the environment.

This understanding of the social determinants illustrates how health is affected by different aspects or scales of one's life, from their neighbourhoods, to their workplaces. This expansive perspective of health, however, was not always intact. There has been a shift towards including wellbeing into definitions of health at the level of organizations. For instance, the World Health Organization (WHO, 2003) defines health as "a state of complete physical, mental and social well-being and not merely the absence of disease or infirmity", where wellbeing becomes an integral part of a person being or becoming healthy. This shift towards wellbeing can also be attributed to the growing influence of the Population Health model. According to the Public Health Agency of Canada (PHAC), "the population health approach recognizes that health is a capacity or resource rather than a state [...] [which] recognizes the range of social, economic, and physical environmental factors that contribute to health" (PHAC, 2012). The PHAC uses specific social determinants to highlight these many dimensions of health for Canadians. There are currently twelve social determinants of health: 
1. Health Services

2. Employment/Working Conditions

3. Education and Literacy

4. Physical Environments

5. Social Support Networks

6. Personal Health and Coping Skills

7. Social Environments

8. Healthy Child Development

9. Biology and Genetic Endowments

10. Culture

11. Financial and Social Status

12. Gender

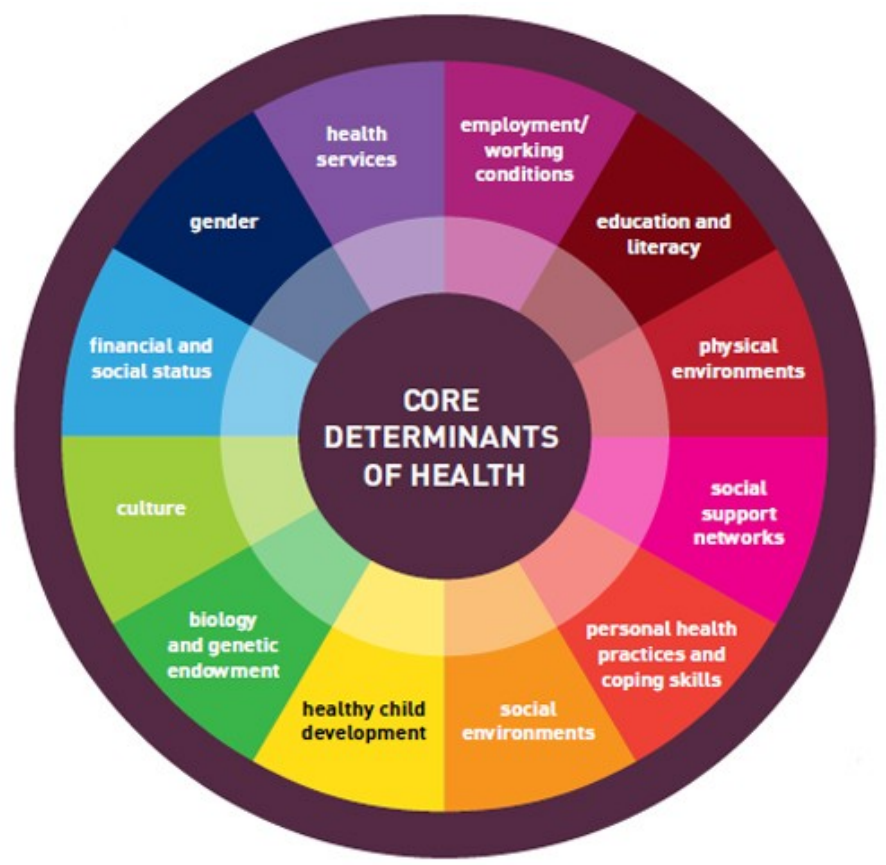

Figure 1: The Social Determinants of Health in Canada

In my research, some of the most

(Government of Canada, 2013)

relevant determinants of health were the role of social support networks and personal health and coping practices. Social support networks refer to the networks, like friends, family, and community people rely or use to better support their own health. There is a particular link to notions of wellbeing in this determinant: "the caring and respect that occurs in social relationships, and the resulting sense of satisfaction and wellbeing seem to buffer against health problems" (PHAC, 2013). Many of interviews highlighted that neighbours and communities played a role in providing health advice and support.

Personal health practices and coping skills were also relevant, and this determinant brings attention to the methods of self-care and the choices people make to maintain their health. One important point to note here is that "there is growing recognition that people's life 'choices' are greatly influenced by the socioeconomic environments in which people live, learn, work and play" (PHAC, 2013). This point highlights the importance in recognizing 
how health is maintained through daily practices or routines but also how these may then be influenced by one's environment

The Social Determinants of Health model is also useful in highlighting health barriers some participants faced. Culture is typically referred to as a major health barrier for newcomers, as many seeking health services may not have access to culturallyappropriate services or may feel discriminated against because of their cultural background (PHAC, 2013). However, Viruell-Fuentes et al. (2012) feel that the perspective of culture proposed here is quite static, when in fact, culture is always changing. There also appears to be an underlying assumption that culture is a problem, with some health services and professionals "inadvertently promoting victim-blaming explanations" (p. 2100). As well, these views "do not account for the notion that structural factors likely impact health in broader ways than simply influencing a culture's orientation" (p. 2100). Barriers, such as discrimination, racism and racialization, as well as segregation are factors which need to be considered, rather than painting culture in such a broad stroke, as a major barrier to accessing health care.

In addition, literacy and unemployment also came to be prominent determinants in my research. Because of educational attainment unrecognized in Canada, as well as some participants not being fluent in English or French, barriers to employment occurred. These determinants, however, do not recognize other facets of employment or literacy. For example, there is little attention to how not having a job can cause a person to become ill through stress. As well, little has been said on how notions of literacy can be related not only to educational attainment but to one's ability to navigate health services, a concept called health literacy. WHO (2009) defines health literacy as 
the cognitive and social skills which determine the motivation and ability of individuals to gain access to, understand and use information in ways which promote and maintain good health. Health Literacy means more than being able to read pamphlets and successfully make appointments. By improving people's access to health information and their capacity to use it effectively, health literacy is critical to empowerment.

Attention to how barriers to health services in conjunction with literacy is an important determinant to consider for migrants who do not speak either of the official languages, Nutbean (1999) writes that "the traditional definition of health literacy misses much of the deeper meaning and purpose of literacy" (qtd. in Nimmon, 2007, p. 337; cf. Nutbean, 2008) outside of the education system. Works in related fields, such as public health, are also primarily concerned with definitions, which are typically quantitative in nature (Rootman and Ronson, 2005; Zanchetta and Poureslami, 2006; Omariba and Ng, 2011). Through defining health and wellbeing in this research, it was also found that health literacy played a role outside of people's experiences in accessing health care services. Other researchers, like Simich $(2009$, p. 4) insist that "enhancing health literacy applies not only to medical settings, but also to a variety of everyday settings and across the life course". Nimmon (2007a, 2007b) echoed much the same point but used photonovellas and ethnodramas to highlight how participatory methods could be used to reveal lived experiences and definitions of health literacy for immigrant women.

Chiefly, migration was a major determinant of health in this research. This has been explored by the University of Toronto's Faculty of Nursing, in research on new immigrants in Ontario (Gastaldo et al., 2005), undocumented workers in Ontario (Gastaldo et al., 2012), and Brazilian immigrants in Ontario (Magalhães et al., 2009). In each of these projects, different aspects of migration and settlement are highlighted, showing the nuanced ways in which migration affects one's health in daily life. 
Moreover, critics like Raphael, have also argued that other determinants should be added to the existing twelve. For example, Mikkonen and Raphael (2010) list fourteen, with new determinants, such as unemployment and job security, income and income distribution, social exclusion, Aboriginal status, disability, and race. Their inclusion of other identities, like race and disability, can highlight other nuanced experiences associated with health. In my research, I found that attention to the stories and lived experiences Nepalese and Bhutanese people shared with me, were the best way to understand health and wellbeing. The main goal of this research, then, is to use these stories, these narratives, as a means to better health and wellbeing from the perspective of Bhutanese and Nepalese people, to examine how these notions can be seen as placebased, with attention to daily routines. In all, demonstrating the 'everydayness' of health and wellbeing may be the first step in identifying important determinants of health, to provide health services better catered to people's needs.

\section{Framing "Health" and "Wellbeing"}

When this research was conducted, the terms 'health' and 'wellbeing' in the interview questions were framed in a direct way, so that questions connected these terms to one's daily life and daily practices. Attention to everyday places and practices was important to this research, as it is there where knowledges interact and influence the types of practices which become associated with health, wellbeing and healing (cf. Dyck, 2006). Notions of health and wellbeing varied in the responses. Some participants felt health was a task that one maintained on their own, while some saw health related to their body aging. One participant connected her definition of health to broader notions of 
biological health and genetics, suggesting that one can be healthy or unhealthy not only through the way they lead their life but also through genetic dispositions, based on race.

The variety of responses illustrated to me how health could be conceived as a lived experience, unique to each person. As well, it is important to consider these accounts as placed, and I argue that attention to accounts of health and wellbeing need to be linked to place. Health geographers, like Dyck (1995a, 2006) have noted that health can no longer be understood as simply the absence of disease or illness but as connected to other parts of one's life, like employment, family and culture, and this expansive view of health is also evident in the definition proposed by the WHO (2003) mentioned previously. Paying attention to how people define health in relation to the many facets of their life is crucial here. Others have has shown how health and place can interact on the scale of everyday life, and that attention to these spaces can make these connections more apparent. for instance, Dunkley (2009) has shown that links between health and place are not always material but can be based and maintained through practices. To this, Palmer (2012) writes that "the need to understand that an individual's emotional relationship with a certain place is informed and created by social relations and practices enacted in them, physical territories may be capable of being made and unmade as sacred or meaningful because of the socio-ritual activities enacted there" (p. 72). Through Palmer, it is clear that it is not always enough to listen to the accounts people share about health, it is also important to place these accounts in their spatial, as well as social contexts.

With discussions on wellbeing, a variety of responses were collected, with wellbeing seen as linked to one's emotions, wellbeing as an expansive form of health, that included nurturing the wellbeing of one's family and sometimes one's community. Much 
like the WHO definition of health above, I framed notions of wellbeing as related to health, and my understandings of wellbeing came from health geographers who saw wellbeing as the experience of 'being well' (Kearns and Collins, 2010, p. 20), and those who viewed wellbeing as a journey towards health (Healey-Ogden and Austin, 2011). Here, wellbeing was seen as the experience of being healthy, health's emotional counterpart. Researchers, like McMichael and Manderson (2004) have used wellbeing as a lens to explore notions of nostalgia among Somali refugees in Australia. Attention to their nostalgic accounts can reveal much about their present situations during settlement, as relational rather than synonymous, can reveal how both terms are also place-based, geographical concepts and phenomena.

\section{Objectives of the Research}

This research has three main objectives. The first to identify how Nepalese and Bhutanese people define 'health' and 'wellbeing' in the context of their everyday lives and routines. Understanding health in the context of daily life has been explored in health geography literature, and in particular, Dyck (1995b) has written that "lives are lived in specific spatio-temporal settings that society and the individual recursively constitute" (p. 308). Attention to these daily routines, like cooking, going for walks or socializing in one's community or neighbourhood can influence how one maintains their health. In order to expose these links, I have opted to use semi-structured interviews as a way for participants to explain in their own words what health and wellbeing mean to them.

Another important objective is to highlight links between health and place, through an application of relationality. Cummins et al. (2007) acknowledge that people's 
relationships to place change over time, and I feel that migration and settlement are two major life events that can affect how people relate to place. As well, the authors recognize that places are constructed and maintained through activities and actors. I use the relational approach to highlight how everyday routines can be read as place-making activities. I feel attention to these practices can better highlight how health and place relate to and define each other, through interviews with participants.

The last objective I plan to meet in this research is the attention to the experience of conducting research. In the context of qualitative research, social scientists have written extensively on the concept of reflexivity, which refers to the critical process of a researcher examining their role in and thoughts about their research. However, I would like to draw attention to the uncomfortable moments of my own reflexivity. To do so, I point to moments of difficulty and highlight the many dimensions of reflexivity, though written entries in a field journal I kept.

\section{Outline of Chapters}

This thesis is organized into five chapters. This first chapter introduced the background and context of the research, by outlining my motivations and reasons for researching this topic. I also highlighted the historical context of Nepalese and Bhutanese migration routes to Canada, and briefly touched upon some of the issues the Bhutanese population has experienced post-settlement in Canada. I introduced dominant perspectives of health and wellbeing, through the Social Determinants of Health, and noted those evident in the research findings. Through the research objectives, I mentioned the particular important aspects of the research, namely, my attention to the importance 
of participants' narratives about health and wellbeing, using a relational approach to expose links between health and place, as well as critically examining reflexivity through my research.

Chapter two is an overview of my theoretical framework and review of the literature. I first explain postcolonial theory, referring to key theorists, like Edward Said, Frantz Fanon and Stuart Hall, and I then highlight how postcolonial theory has been used in geographical works. My use of postcolonial theory is centred around debates on postcolonial knowledges, and attention to other forms of knowledge outside the text, mainly through stories or narratives. Next, I move into my review of the literature where I discuss the definitions of place in medical and health geography, then turn to my own rendering of place in this research, influenced by Cummins et al.'s (2007) relational approach in health research. After situating place in this research, I then move into unpacking the different bodies of literature relevant to this research, namely geographical works related to disability, care geographies, and works exploring the everyday health geographies of immigrants and refugees. This review is meant to explore how health and place interact in the literature.

Chapter three discusses the methodologies used in the research. I highlight my reasoning behind using feminist methodologies, with their particular attention to power relations, my positionality or role as a researcher, subjectivity, and the emotional aspects of research. I also pay particular attention to critically examining my research experience through episodes of research failure, through the concept of reflexivity. With this foundation, I turn to my rationale of using semi-structured interviews, discussing some of the strengths and issues to consider. I end with a discussion of the ethical issues of data 
collection, and my mode of data analysis.

Chapter four outlines the findings from the interviews that were conducted with ten Nepalese and Bhutanese participants. From the data, I pulled out five themes: i) definitions of health and wellbeing; ii) attention to walking as a health practice; iii) an examination of cooking as a health and cultural practice; iv) highlighting links between notions of work and wellbeing; and v) roles the neighbouring community play in defining and influencing health. Participants noted varying conceptions of both health and wellbeing, which ranged from the importance of family, the self and hobbies as a way to maintain both. Attention to walking and cooking revealed that both practices related to different aspects of health and wellbeing, such as walking as a means of transportation but also a way to keep one connected their community. For cooking, participants noted the importance of cultural food staples in not only balancing one's diet but also revealed a sense of wellbeing cultivated through the practice of cooking. Discussions surrounding work revealed a strong relationship between having employment and one's wellbeing and self-esteem. The last theme is particularly important to highlight how therapeutic networks can be used as a way to better understand how health can be influenced by one's social networks. Interviews revealed both therapeutic and untherapeutic qualities to varying social networks related to health.

Chapter five concludes with a summary of each of the thesis chapters, beginning with a reintroduction the review of the literature, with my attention to literature discussing the concepts of health, wellbeing, and therapeutic networks, while also referring to the literature from disability studies, care geographies and works related to everyday geographies of immigrant and migrant health. I then move into re-discussing 
the methodological frameworks, highlighting the main findings and themes from the data, and noting the limitations of this study. I end with a final discussion surrounding reflexivity in this research, while also providing suggestions to future graduate students regarding research. I then close with some methodological and theoretical suggestions for future health geography work regarding immigrant and refugee groups that may be worth expanding on. 


\section{Chapter 2: Theoretical framework and review of the literature}

\section{Introduction}

The main objective of this chapter is to demonstrate how health and place can be understood in the context of everyday life, as well as relationally, occurring at multiple scales and networks (Cummins et al., 2007). To achieve this research objective, this chapter will be split into six parts. First, I will discuss postcolonial theory in geography

briefly, then turn my attention to postcolonial knowledges as the theoretical framework in this research. From theory, I will define my use of place in this research, referring to health geography works that share a similar application. As well, I will highlight what it means to use a relational approach in health research, and outline some of the ways in which I plan to use relationality. The third part of this chapter will move to an overview of the main concepts used in my research, namely health, wellbeing and therapeutic networks. Fourth, I will review the relevant literature on everyday geographies through the lens of health, beginning with a focus on disability studies in geography, particularly in relation to concepts, such as the everyday, place, and the body. The fifth section of the chapter will explore care geographies, with particular attention to those works with an interest in examining care as performance or practice, as well as care as an emotion. Finally, the last section of the chapter will focus on works highlighting immigrant and refugee geographies of health.

\section{Postcolonial theory and geography}

A major theoretical framework from literary studies and present in the social sciences is postcolonial theory. Postcolonial theory refers to the examination of the 
historical, political, literary, cultural and economic legacy of colonialism colonization, and imperialism. Postcolonial theory is mainly interested in how power, in the form of text, politics, ideas of dominant knowledge and economy, function to disproportionately affect colonized (mostly non-Western) people of the world. Very early postcolonial theorists came from literary criticism and philosophy, using and deciphering texts as a way to explain the many faces and functions of colonial power. Postcolonial theory now has theorists from various disciplines, though the most well-known of these thinkers is Palestinian-American, Edward Said. In his renowned text, Orientalism (1978), Said deconstructed ideas of the Orient (referring to the Middle East, East Asia and Africa), and the underlying assumptions this carries. Said traced the etymology of the Orient, noting that there are reoccurring notions of savagery, exoticism, backwardness, religious impurity, unintelligence among other negative traits associated with people of this world by European scholars and travel writers. Rather than comment on contemporary conditions, Said noticed that many continue to recycle these age old stereotypes, even off the page. This process was called orientalism, meant to demonstrate the sometimes volatile relationship between knowledge and power. Although Said was interested in exploring how orientalism unfolded textually, he failed to capture (at the time) how orientalism played out on the scale of daily life, as well as how these processes of othering affected those labelled 'orientals'.

Martinique-born postcolonial scholar and psychiatrist Frantz Fanon was interested in the psychological effects of colonialism, mainly due to his work as a psychiatrist in Algeria during the height of the independence movement. In his most well-known text, The Wretched of the Earth (1961), he called for a militant movement of decolonization, 
stating that colonized people should use violence as a means to protect themselves, when needed. Although many sift through the text and only apply the use of violence, Fanon also stressed that colonialism needed to be first decolonized in the mind. In his other text, Black Skin, White Masks (1952), Fanon showed the mental effects of colonialism on the colonized. Fanon was one of the first postcolonial scholars to move away from a textual analysis of colonialism.

With a focus on postcolonial identity and culture, cultural studies theorist Stuart Hall examined the relationship between the two. Rather than focus on the post-colonial as a moment in time, Hall looked at how culture played a role in constructing and maintaining a postcolonial identity in the present. Hall saw cultural identity as always being renegotiated, and wrote that "identities are the names we give to the different ways we are positioned by, and position ourselves within, the narratives of the past" (1994, p. 384). In this sense, Hall's cultural identity wrote against narratives of orientalism. With this introduction to postcolonial theory and some of its renowned scholars, the following section discusses postcolonial theory's relationship to geography.

\section{Postcolonial theory in Geography}

Postcolonial theory and the discipline of geography have a long relationship. Historically, geographers, in the role of surveyors were used to map areas in the developing world for colonization by Europeans. Geographers were crucial partners in the colonial project in much of the world, and helped colonizers better understand the locals. For this reason, postcolonial theory aimed to transcend practices and views said be associated with colonialism, namely "objectification, classification, and the impulse to chart or map" (Sidaway, 2000, p. 592). Furthermore, it is because of this colonial 
relationship that applying postcolonial theory in geographical research can be a sticky affair. Much in tune, Sidaway warns that "any postcolonial geography must realise within itself its own impossibility, given that geography is inescapably marked by its location and development as a western-colonial science" (p. 592). This point, however, does not mean that geographers should forfeit using postcolonial theory in their works but that this limitation first needs to be acknowledged.

Gilmartin and Berg (2007) are also concerned with the relationship between geography and postcolonial theory. With their focus on British geography, they argue that "much for what passes for postcolonial theory in geography reinforces new forms of colonial epistemologies and colonial hierarchies, while destabilizing the older forms" (p. 129). The authors are concerned that there has been little application of postcolonial theory in geography, the specific everyday geographies. The lived experiences of colonialism, as well as postcolonialism need to be accounted for by geographers (p. 122). Mains et al. (2013) echo many of the same points but are more specifically concerned with an application of postcolonial theory in migration geographies. They begin with evidence of the most salient relationship between the two: "there is an explicit link between colonialism and patterns of migration" (p. 131). They remind us of the role of slave labour from Africa to generate economies in Western Europe. However, rather than encourage migration geographies to retell these grand narratives, they encourage geographers to "understand the ways in which migrating bodies have become nexus points for spatial practices across many scales" (p. 132). In this sense, colonialism and postcolonialism are not the main foci but it is migration, the experience of mobility and the lived experiences of migrants which would be the product of applying postcolonial 
theory in migration geography. Considering the historicity of migration, coupled with viewing migration as a very much spatial and embodied experience, postcolonial theory may lend a hand in pushing migration geography out of the 'here' and 'there' sense of place seen in much transnationalism literature (p. 134). In my own work, Mains et al's. points are applicable because it is important to look into the reasons for the migration of Bhutanese migrants, due to religious convictions, and how this may differ from the Nepalese. As well, attention to these lived experiences of the postcolonial through narratives is also useful in my work, examining how migration plays out through the voices of participants.

Much of what contemporary scholars in postcolonial geography seems to be addressing is for greater attention to the lived experiences of postcolonialism. However, what appears to be looked over is the lived experience of being a self-identified postcolonial geographer. Noxolo (2009) calls for postcolonial geographers to re-examine how their body plays a role in representation and postcolonial writing. Noxolo tackles ideas of identity and positionality and how these plays a role in her writing. Defining herself through Gilroy's (1993) notion of the "double consciousness" of being a Black (Afro-Caribbean) and British women, Noxolo recalled her travel to Ghana where her black skin was shared but her understanding of a united Black identity was not. In another excerpt, she noticed a new change in her embodiment during the completion of her $\mathrm{PhD}$ while pregnant and raising young children (p. 59). She returned to her racialized identity when speaking on the few people of colour in geography (p. 59). In these excerpts or snapshots of her academic life, Noxolo demonstrates the intimate geographies of embodiment and identity of postcolonialism. Moving away from larger stories of the 
colonial expansion in her native country in the Caribbean, Noxolo instead began with her journey into academia, then highlighting how self-identity, race and motherhood have all played a role in her life. To understand what counts as postcolonial knowledge in texts, first attention to these intimate geographies of postcolonialism need to be fleshed out. The following sub-section tackles this discussion, to reconsider what counts as postcolonial knowledge.

\section{My application: postcolonial knowledges}

One slowly growing stream of postcolonial inquiry, led mainly by geographer is examining postcolonial knowledges, which is the aspect of postcolonial theory I apply to my own work. As mentioned, many of the most well-known postcolonial theorists, such as Said, were literary critics or philosophers, and as such, their analyses were very much textual. However, to apply postcolonial theory in other fields, like geography, other notions of knowledge need to be acknowledged. One of the first people to heed this call was historian Dipesh Chakrabarty (2000) in his seminal text, Provincializing Europe: Postcolonial Thought and Historical Difference. In his text, Chakrabarty is critical both the discipline of history and postcolonial theory for failing to account for other knowledges and notions of time outside of European influence, discussing topics such as the subaltern, subject positions and identity of formerly colonized peoples.

It was Chakrabarty's work that influenced geographer Robinson (2003) to write a piece about provincializing postcolonial theory in geographic scholarship. Robinson wants postcolonial geographers to think cosmopolitan when it comes to scholarship. Robinson is concerned that "even as the postcolonial turn to those working on and in other places to diversify the 'voices' and 'contexts' in their academic collaborations, these 
different contexts have usually been incorporated as 'case studies'" (p. 278). So-called 'other' knowledges appear to be treated as secondary or supplementary data or information for geographers, and this relationship needs to change to better apply postcolonial theory in the discipline. It is because of this privileging of Western scholarship as primary knowledge that Robinson feels the need to greatly change and "to move beyond this divisive geopolitics of knowledge" (p. 278). Her suggestions, such as encouraging Western geographers to publish in lower-tier journals or e-journals to make their works more accessible (p. 281), and to regionalize the discipline by using literature from other parts of the globe (pp. 280, 281) are enlightening but somewhat naïve. I feel Robinson has not greatly considered how new geopolitics can occur, with Western geographers likely favoured over geographers trained in non-Western countries in the case of publishing. As well, discussing how individual researchers could better apply these other knowledges in their own research should have also been explored.

Jazeel and McFarlane (2010) also touch upon the importance of multiple postcolonial knowledges in their piece. They discuss the importance of responsibility in postcolonial research, encouraging postcolonial geographers to reconsider "knowledge's politics" (p. 110). They hope that researchers consider how knowledges are not free from politics and power. In addition, as geographers, we should also understand how "knowledge produces place" (p. 113), and that researchers can encapsulate participants to particular places while situating themselves to others, a process that if not recognised, can re-produce orientalist assumptions. To counter this, Jazeel and McFarlane encourage postcolonial geographers to understand the concept of wordling. They write that "learning is a process of wordling [...] it is formed in the world, in the distanciated 
ecology of interactions between texts, people, sites and objects; and is of the world, in its capacity to represent different people/sites" (p. 115). This idea of wordling is useful in my application of postcolonial theory because it pushes it to move outside of merely focusing on the text, and to consider places, peoples and objects, all in interaction. Wordling is also intriguing because it recognizes that knowledges change in new environments (p. 116). In relation to my work, ideas of health and wellbeing change in the face of migration and settlement in Canada, showing that health and wellbeing are very much relational concepts. The authors end with a warning that "all intellectual projects have geographical imaginations, and these imaginaries have real world effects" (p. 122). In the case of migrant health, not recognising various understandings of health and wellbeing can be the difference between life and death when treatment is needed.

One minor issue with the works noted above is how this can be applied theoretically and methodologically to geographic research and research settings. McLees (2013) comments on this theoretical-methodological gap in postcolonial research in geography (see Raghuram and Madge, 2006), and suggests that postcolonial theory steer from texts to the everyday lives of participants. Through examining urban farms in Tanzania, McLees believes "postcolonial methodological approaches are needed to illuminate how these daily realities create and influence a different kind of city" (p. 284). McLees proposes using methods like participatory methods, like PhotoVoice, as well as traditional qualitative methods, like semi-structured interviews, to listen closely to responses and as a way for participants to speak for themselves. Through these methods, McLees believes that researchers can understand postcolonial realities, as well as hidden neocolonial influences in daily life. In my research, semi-structured interviews were used 
to do just this, with particular attention to the role of migration in shaping notions of health and wellbeing.

Other scholars have noted different ways of accounting for postcolonial knowledges. Besio's works $(2003,2005,2006)$ call for the use of story-telling and autoethnography to foreground participants as active partners in qualitative, transcultural research. In one particular piece (2005), Besio does this eloquently, showing that postcolonialism can be understood in northern Pakistan through the idiom of sewing machines. As well, Besio feels that through story-telling, the effects of colonialism can be better highlighted and explored. In this article, the author connects women's stories of sewing to wider stories of the tourism industry in villages of northern Pakistan. Besio questions what counts as knowledge, especially in her work with women, as many in the region feel voiceless. Attention to so-called smaller stories in this context can reveal new geographies of postcoloniality, especially for women. Besio does much the same in a later piece (2006), through the idiom of chutes and ladders, used to climb house structures in Askole villages in Pakistan. In this piece, Besio demonstrates that this mobility writes against grand orientalist assumptions of female subjugation, as well as in local jargon, with the Hindi and Urdu term, jangli, meaning 'dirty' or 'of the jungle' (p. 262). In the face of cultural practices, such as $\operatorname{purdah}^{1}$, the neocolonial tourism industry, and militarization in the region, Besio tries to show how "Askole subjects use space to assert their agency in strategic ways" (p. 262).

Marte's (2011) use of the term seasonings functions as a way to place together both colonial and postcolonial identities for Afro-Dominicans in New York City. With her interest in ethnic and racial food maps and geographies for Dominican migrants, the

\footnotetext{
${ }^{1}$ Female segregation, typically through the use of walls, curtains, or face coverings
} 
term seasonings is meant to "point to the struggles, movements and wider contexts of memory-history that made possible the present relations between these people, food and places" (p. 181). These seasonings, such as recipes for Dominican foods, like mang $\dot{u}^{2}$ are evoked with pre-migration experiences and memories of cooking. For postcolonial theory, the narrations of migrant memories serve an important outlet for situated knowledges that are both 'here' and 'there' but also an example of place-making. Furthermore, these seasonings do not only apply to the past but also as a referent to the present, the present is just as important. In her work, Marte takes notice of the food choreographies that Afro-Dominican migrant women perform daily: "migrants produce their own reality of the city through their embodied experiences as they participate in the pulse of the city" (p. 188). In other words, the everyday practices of food-making of migrants should be of importance to postcolonial geographies, as they reflect both a life lived elsewhere weaved into daily practices in the diaspora.

Others have also focused on the importance of memory as knowledge. ToliaKelly (2004a; cf. 2004b, 2004c) considers memory as knowledge in her work with South Asians living in the UK. With particular attention to postcolonial and cultural geographies through the lens of material culture (cf. Nash, 2002; McEwan, 2003; Cook and Harrison, 2003), the author feels that memories of India, Pakistan and East Africa are contained in objects in the home that resemble their notions of 'back home', and the concept of re-memory is used to make this connection between memory and object. toliaKelly explains that re-memory refers to the "encounters with memories, simulated through scents, sounds and textures in the everyday" (p. 314). Much like with Marte's

\footnotetext{
${ }^{2}$ Traditional Dominican dish of boiled and mashed plantains, topped with fried red onions, and occasionally served with fried eggs, salami or avocados as sides.
} 
(2011) work, memories function as postcolonial knowledge, informing the present with glimpses of the past: "sensory memories are shards of other environments, evoked in the practices of everyday living collaged together as a landscape of nostalgia, but also as a memory history of Imperial landscapes" (2004b, p. 290). Closely examining the role of memory, especially nostalgia, is useful in demonstrating how knowledge takes different forms, as well as how it shifts depending on the environment. Overall, this section demonstrated the many knowledges that can be included through a postcolonial lens. In my work, I hope to take into account the narratives and practices of health maintenance as evidence of knowledges integral for a greater understanding of health and wellbeing in the lives of Bhutanese and Nepalese participants.

\section{Place in medical and health geography: discussions and applications}

Theorizing and unpacking place is an important foundation to geographic research. First proposed by Agnew (1987), Cresswell (2009) sees place as being composed of location, locale, and sense of place. Location referring to 'where' places are; the locale, which is its material setting; and sense of place, referring to people's emotional reactions to places deemed significant to them (p. 169). Cresswell also notes that what makes places different from spaces is a combination of materiality, meaning and practice. Places may be material, in the form of physical, visual landscapes or structures. However, places are also important for the meaning people ascribe to them, as "meanings can be very personal and connected to individuals and personal biographies [...] but meanings are also shared and in some important ways, social" (p. 169). This attention to 
meaning is important to this research, as people's understandings of health and wellbeing can inform which places they deem significant. The last layer of place Cresswell mentions is how places can be practiced. More than just the location of practices, spaces also become places once practices are regularly performed and given meaning by people (p. 170). It is these two components of place, meaning and practice, that are important foundations to my understandings of place in this research.

In addition, I situate my understanding of place in the wider discussions of the shift from medical to health geography in the early 1990s. These discussions were centred around debates of a 'new' medical geography, interested in examining links between health and place. This new medical geography critiqued quantitative works done by earlier medical geographers, primarily related to disease ecologies. There was also a question of an undertheorized space, little distinguished from place (see Kearns 1991; Kearns and Joseph, 1993; Kearns and Moon, 2002; Litva and Eyles, 1995; Moon, 2009; Rosenberg, 1998). Specifically, these debates about place began with a call from Kearns (1993) to other medical geographers to help develop a post-medical geography, a linking of medical geography to social and cultural geography within post-modern attempts to account for difference. However, some geographers were concerned with Kearns' lack of attention to difference in the form of the body (Dorn and Laws, 1994; Hall, 2000; Parr, 2000, 2002), while Mayer and Meade (1994) argued that other ecological models of medical geography focused on disease should not be ignored in favour of this new medical geography. Much later, Andrews and Evans (2008) highlighted some of the issues found with the term, post-medical. One was that "medical concerns still arise in many contexts related to health, and cannot be separated or easily ignored" (p. 763). 
Another was that "post-medical reactionary rhetoric is based on a simplistic and dated caricature of medicine as something universal, universally powerful and uncaring" ( $\mathrm{p}$. 763). I understand their points to mean that some medical geographers during these early debates placed the 'medical' and 'health' in diametric opposition, turning the medical into anything 'health' was not. These two concepts would need to be better flushed out and contextualized in the research to better highlight why a new medical geography is necessary.

Nearly a decade after these debates began, Kearns and Moon (2002) discussed the changes in direction that medical geography (now called health geography), has taken since its inception. This new health geography has turned to new conceptions of place, viewing place as the location of health geography research, as well as seeing it as placeknowledges, bringing participants' voices into the research (p. 610). Kearns and Collins $(2010$, pp. 17,18$)$ noted that in health geography today, place is conceptualized in three ways: i) humanist traditions of examining one's place-in-the-world, which has been readily taken up by feminist geographers, and researchers in disability studies; ii) literature on therapeutic landscapes, the particular landscapes associated with health or containing supposed health-giving properties (cf. Gesler, 1992); and iii) research using multilevel models to examine links between health and place, with existing quantitative data.

\section{Renderings and applications of place}

My use of place in this research is informed by the idea of emplacement ${ }^{3}$ as a way to account for the lived experience of health and wellbeing. With the three schools of

\footnotetext{
${ }^{3}$ I take Casey's (2001) description of the geographical self as a way to define emplacement, as the self and place fold onto each: "there is no place without self and no self without place" (p. 684).
} 
place in health geography noted above, my use of place falls between the first two, as an understanding of place as a lived experience that can also be captured through daily routines, which in turn create these healthy places. Below, I highlight some works that I feel are relevant in bringing this forth.

Kearns and Joseph (1993) defined place as "anchored in the world of human experience" (p. 712), and these lived experiences can take place in various everyday spaces, making them place-based. Dyck (1995a) expressed a similar view of place, as "the experienced collectivities of concrete spaces which are occupied, used and given meaning in the course of interaction in social life" (p. 308). These places, then, respond to and are implicated by social interactions. My rendering of place is most evident in works by Dyck. In a piece on chronic illness and health through the eyes of Chinese and South Asian women (Dyck, 1995a), place is defined "as dynamic, constituted and reconstituted through the operation of uneven distributions of power over space, with individuals interacting and experiencing place in different ways as they engage in various sets of social relations" (p. 248). This view of place illustrates a connection to one's social positioning in society, which shows how people experience and define places differently. Dyck feels that one's position in society, as well as the daily routines one performs to maintain their health all play a part in how place is defined and experienced. In the following section, I will refer to a relational approach as a better way to sync health and place in this research.

\section{Relational approaches to health and place}

Mentioned in the introduction, one of the main objectives of this research is to better connect health and place. Using a relational approach to understand health is a way 
to illustrate these many shifting dimensions of health in place. Cummins' et al.'s (2007) idea of a relationality recognizes that health takes place along various networks, and they define the relational approach to place in several ways. First, there is the importance of recognizing "the dynamic and changing character of places and place-to-place mobility of populations on a daily basis, and over the life-course. This implies that individuals often influence and are influenced by, conditions in multiple places" (p. 1828). This point is very important, as it recognizes that place's importance to health can shift depending on changing health practices. This place-based approach can also account for changes in perceptions of health throughout the life course, as well as when one's geographic location changes. Migration, for example, is a major factor to consider when linking health and place, as both may then become multi-scalar: "places are produced and maintained by the activities of 'actors', proximate or distant to a particular place, who operate individually or in concert across a wide range of geographical scales" (Cummins et al., 2007, p. 1828). This idea of place as multi-scalar can also expand ideas of health, making room for health to be stretched across borders. In the context of my own research, I suggest that ideas of health are not only influenced by one's immediate environment but also environments prior to migration. Using a relational approach can also be useful in viewing health as more than a measurable behaviour, a step away from earlier and more prevalent conceptions of place effects. Macintyre et al. (2002) feel that the term place effects have not been adequately unpacked, and "have the status of a residual category, an unspecified black box of somewhat mystical influences on health which remain after investigators have controlled for a range of individual and place characteristics" (p. 125). This point is one reason that the use of relationality in understanding health and place is 
crucial because it is based on examining how relations with both the human and nonhuman world play a part in our health.

Research regarding health promotion, as well as policy, are beginning to make relational connections. In the UK, greater focus on the role of community care has encouraged an interest in how place and health interact. Martin et al. (2005) examined how attention to place in health settings can have a major effect in how health is understood, experienced, and achieved. In this work, some respondents noted feeling more healthy and cared for in the home environment as a place for care, while for others, it was in the institutional space of a hospital or rehabilitation centre. Although clearly indicating how place and health are understood differently among individual patients, the authors felt that "what emerges is the centrality of social and symbolic contexts in determining the therapeutic qualities of these different settings of intermediate care" (p. 1901).

Similarly, Burges Warson et al. (2007) looked at the relationality of place and health when considering maternity care and labour pain. The authors used the term flexible therapeutic landscapes. Flexibility in this work appears to be interchangeable with the notion of relationality, as it describes how "flexibility in provision creates a space for women's preferences to be heard, and for discretionary practices in the interactions between health care practitioners and women in labour" (p. 866). Again, the theme of relationality is present here, as these experiences, the physical and symbolic features of the space, as well as interactions with hospital staff, all influence whether a space is rendered as therapeutic for each individual pregnant woman. This work comes on the heels of changing policy in the UK regarding maternity care, with the intent that 
the policy will focus its attention to where pregnant women feel empowered in the decision to choose where to give birth; this is another dimension of the authors' notion of flexibility. Burges Warson et al. call for "a relational view of place that takes into account how women's sense of place may modify that sense of place, creates opportunities for making more out of the therapeutic qualities of particular settings" (p. 874). In sum, a relational approach can better account for health and place's relationship in the face of changing health policy.

\section{Overview of conceptual frameworks}

This section will unpack the main research concepts: health, wellbeing and therapeutic networks, by illustrating and commenting on how geographers and those from related disciplines have used these in their own work, and I will then explain my application of these concepts in my research. Overall, the main aim of this section is to demonstrate how these concepts could be applied and situated in the context of the everyday lives of migrants.

\section{Health}

In the last chapter, I noted that the influence the Population Health model in shifting attention from biological notions of health. There needs to be a consideration of power possibly veiled in definitions of health, as "health is, to a large extent, constructed by the health care systems prevailing at any particular place and time" (Gesler and Kearns, 2002, p. 7). In this thesis, I am interested in demonstrating how health can be affected by a multitude of influences in one's life. Indeed, Gesler and Kearns (2002) have shown that "health arguably allows an expansiveness that blends the cultural, 
environmental and social with greater ease" (p. 6). They find this expansiveness in the etymology of health, from the old English haelth, "which involved three meanings: whole, wellness, and the greeting 'hello"' (p. 6). Moving away from a strictly biological and medicalized definition of health can reveal these nuanced origins of the term. In health geography itself, discussions on health have also changed. Kearns and Collins (2010) feel this is a result of the prevalence of the Population Health model, which highlights the importance of "wellbeing as capturing more positive components of health, still seen as associated with illness and disease" (pp. 18, 19). Interest in wellbeing may also be a way to escape clinical and biological underpinnings in the word health "used in popular discourse, as a synonym for medical care/intervention" (p. 27), as well as a way to link back to the origins of the term, as noted. This gap that Kearns and Collins suggest is also evident in the literature, where health is not necessarily unpacked. In other words, health geographers have shied away from defining health, and have moved towards examining wellbeing in their works. Nonetheless, in the literature to be reviewed, health was found to be conceptualized in two ways: health as balance, and health linked to culture, religion and spirituality.

\section{Health as balance}

Viewing health as balance is not a new perspective, and as noted in the introduction, is even highlighted in the World Health Organization's (2003) definition of health. This view appears to carry over in this literature, where health was something to be balanced with other spheres of one's life, using a phenomenological lens. For example, Lipworth et al. (2011) found balance to be a noun, the state of balance of one's physical and psychological health (p. 716), which also referred to having a sense of balance in 
multiple spheres of life at once (p. 717). Ebrahimi et al. (2012) also explored this idea of balance, through interviews with older adults. They found that for participants, having balance meant being able to adjust and perform one's daily routines. As well, the body played a role in these narratives, as "the experience of the body working by itself, in both the physical as well as cognitive aspects, was a prerequisite for the experience of good health" (p. 1517). This functioning body included good vision, body balance and mobility, as well as sharp cognitive abilities (p. 1517). Lastly, having balance meant being content or emotionally satisfied with one's life in general. This meant having a strong sense of self-worth, as well as being involved socially in one's community (p. 1518). Ebrahimi et al. have shown how health, through balance, can affect different aspects of one's life, including their own emotional state but also relationships with others. Wagman et al. (2011) found much the same rendering of health as balance in their work in Sweden, where balance was also connected to the body, one's social relationships and cognitive functions, as well as the balancing of one's time. Overall, these works have shown that being balanced or healthy meant having a sense of control in managing one's body but also having the opportunity to enjoy social relationships and daily practices. Greater attention to ideas of place in narratives of health and balance would be a great contribution health geographers could offer.

\section{Health as linked to culture, religion and spirituality}

Some health geographers have considered the role(s) culture, religion and spirituality play in perceptions of health. For example, Gesler's (1992) concept of therapeutic landscapes was popular for its attention to religious healing sites as possessing therapeutic properties, such as Epidauros, Greece (1993) and Lourdes, France 
(1996). However, his works did not pay attention to how everyday practices of religion may also influence people's ideas of health. Wilson (2003) examined Aboriginal ideas of health through a critique of therapeutic landscapes, as conceptions of these therapeutic landscapes are predominately Western, with little attention the therapeutic qualities in other cultural groups, especially in everyday life (p. 84). For the Anishinabek, health was understood as a balance between the physical, emotional, mental and spiritual, all corresponding to the four colours and four directions of the medicine wheel (p. 87). As well, health was very much connected to one's relationship to the land, through the use of traditional medicine, as well as rituals, like the sweat lodge. Dyck and Dossa (2007) found religion to play a role in how health was understood by Punjabi Sikh and Afghan Muslim women in Vancouver, mainly through daily prayer. Manning (2012) found strong links between spirituality, health and old age, showing that through spirituality, many older adults felt a sense of resilience or strong during stressful times in their life, like the loss of a spouse. Similar to balance mentioned previously, health was again expressed relationally, through one's cultural background and religious practices.

Other works have challenged these connections between culture and health. For example, Madge (1998) used therapeutic landscapes as a way to understand how herbal, indigenous and biomedicine interacted in daily life by the Jola in the Gambia, and highlighted attention to these various networks of health care, including religion. This work is significant in demonstrating that cultural perspectives of health can be connected to wider structures of power and political economic realities. Madge stresses that "health care systems do not simply develop in response to particular cultural practices embedded in particular places by particular groups of people, but they also develop in the context of 
unequal political and economic realities" (p. 309). This point is evident in McMullin's (2005) ethnographic research with native Hawaiians, where cultivating a 'healthy' Hawaiian identity was linked to retaining one's cultural identity. This notion of health was meant to rewrite public health perspectives of Hawaiians as unhealthy, with little understandings of what health means to many Hawaiians, as well as contextual reasons as to why this may be the case. McMullin noted several practices said to go in hand with being a healthy Hawaiian. One involved eating a Hawaiian diet, rich in freshly caught fish, and indigenous foods, like taro. Another was having access to one's land, especially in the face of urbanization and the tourism industry expanding on indigenous lands. Finally, having a connection with one's ancestors was also said to make a person healthy, as ideas of health were also connected to a pre-colonial Hawaiian lifestyle. This notion of a healthy Hawaiian illustrates that cultural conceptions of health involve more than attention to rituals or food but also how wider processes, like colonialism, tourism and urbanization can prevent people from achieving a desired sense of health. Culture appears to influence health in myriad ways in these pieces.

\section{Wellbeing}

Wellbeing has been the centre of much debate and change over time. MacKian's (2009) entry for the International Encyclopedia of Human Geography traced the trajectory of the term wellbeing in Western societies, beginning with the Greek word, eudaimonia, meaning 'happiness', to the state's responsibility in keeping its citizens happy (cf. Atkinson and Joyce, 2010). MacKian also noted that recent developments in conceptualizing wellbeing have now focused on concerns regarding welfare (p. 236), wellbeing as part of individual choice (p. 239), and more recently, as part of an industry 
catering activities, like yoga or Tai Chi (p. 239). Sointu $(2005,2006)$ found much the same in research related to discourses of wellbeing in British society. In one piece (2005), Sointu felt that ideas of wellbeing could be connected to changes in subjectivity, that is, wellbeing was connected to being a self-responsible agent or consumer. This agent was also tied to wider societal understandings of wellbeing, from "an issue pertaining to the 'body politic' in the mid-1980s, it now appears to have become a question solely related to the 'body personal"' (pp. 255-256), all connected to an increasingly consumer-driven society.

For health geographers specifically, the publication of Wellbeing and Place (Atkinson et al., 2012) was a major call to health geographers to reconsider and reframe wellbeing in their works. In this text, the authors of the various chapters examined the notion of wellbeing, for example, through the lens of urban greenspace, the neighbourhood, and Aboriginal identity. For this review of literature, I am interested in the contributions that focus on wellbeing as it occurs through everyday life. Broadly, to Kearns and Collins, "the ongoing challenge for health geographers is to rethink the issue of wellbeing by contextualizing it into both the personal and population-based experience of place, while holding firm to traditional concerns for equity and social justice" (2010, p. 27). The following sections will examine some changes in the definition of wellbeing, then turn to literature revealing connections between emotional and economic perspectives of wellbeing, and then end with works discussing cultural perspectives of wellbeing. 


\section{Wellbeing: debates, definitions and distinctions}

The term wellbeing is seldom problematized, semantically. It is at times seen as a component of health, and other times, made synonymous with quality of life, interested in the ranking of countries and city, primarily based on material aspects of life (Kearns and Collins, 2010, p. 20). Flueret and Atkinson (2007) highlighted some of the issues in applying wellbeing in health geography research, arguing that there needs to be a push away from policy applications of wellbeing to better consider what wellbeing means in different contexts. To situate their discussion, Flueret and Atkinson divided wellbeing research in geography into four streams: i) spaces of capability or self-fulfillment, ii) integrative spaces, iii) spaces of security, and iv) therapeutic spaces or landscapes (p. 113). What is stressed in this piece that to best define and conceptualize wellbeing, there needs to be an understanding that "relationships which are complex and two-directional: settings can influence health-related wellbeing, and health-related wellbeing can influence settings" (p. 113). In other words, this model is mean to highlight complex relationships between different notions and places related to wellbeing. Some of the suggestions they leave for future researchers is to consider the use of case studies, as well as ethnographic methods to better demonstrate contextual and cross-cultural understandings of wellbeing (pp. 114, 115).

Healey-Ogden and Austin (2011) explored how terms such as health, wellness and wellbeing are little differentiated. The authors state that "the word wellbeing emerges in the results of research about mental health, self-transcendence, and the lived experience of health" (p. 86). Although this particular piece was not done with participants who had migrated to the country, the authors do mention that the "study 
reveals that well-being lies on the journey through life events when people embrace the journey wherever it takes them" (p. 93), demonstrating how notions of wellbeing can change in different contexts and geographies of one's life. Other relevant works discussing wellbeing include Harvey's (2014) article on understandings of health and wellbeing for women living in isolated Queensland, Australia, as well as Doran's (2014) piece, which explored ideas of wellness within illness, in the context of chronic pain.

Prominent health geographers Gavin J. Andrews and Robin Kearns have called for a greater theorization of wellbeing in health geography. Andrews et al. (2014), like my own work, see wellbeing as emerging in everyday environments. However, what is different about their approach is their use of Non-Representational Theory (NRT), which they define as the mobile energies before and between human and non-humans, wherein wellbeing would be the result of these interactions "experienced as a feeling state" ( $p$. 211). In other words, in wellbeing through NRT, it is these mobile energies, not humans or their surrounding places that have a role to play in wellbeing, reducing agency. In opposition to their work, Kearns (2014) felt that Andrews et al. did not consider the role of the self in these affective understandings of wellbeing. Kearns suggested the use of auto-ethnographic, as well as arts-based approaches, like poetry, to better understand the lived experience of wellbeing (p. 148). With these views and debates in mind, the following sections will detail various camps of the study of wellbeing.

\section{Socio-economic and emotional perspectives of wellbeing}

Conradson (2012) found studies on wellbeing as present in the sub-disciplines of health, emotional and economic geographies. This section will examine the latter two in tandem to reveal how wellbeing can be intertwined with various aspects of daily life, like 
work and cultural beliefs. To illustrate, Dean and Wilson (2009) incorporated emotional, as well as economic perspectives of wellbeing in their work, and found major links between the inability to gain employment for recent migrants, and their mental health. One example of this link "is through what immigrants described as a loss of employmentrelated skills. This process has been deemed de-skilling, which refers to the loss of skills and knowledge that accompanies under/unemployment among immigrants" (pp. 193194). This view of wellbeing is especially important considering the target population I am working with, many of whom are on government assistance. Simich et al. (2010) mentioned similar points in their work with Sudanese refugees, suggesting that both mental health providers and researchers needed to pay attention not only to the trauma and mental disorders resulting from pre-migration experiences but also to everyday postmigration realities (pp. 199-200). These everyday realities included lack of social support networks, marital and parent-child conflicts, lack of education, and employment. It is clear then, that wellbeing is a web of these different facets of one's life: social, familial, cultural and economic. Simich et al. were interested in connecting ideas of wellbeing with home-making in the diaspora, as a way to "enlarge the perspective of refugee mental health to examine qualitative meanings of home reveals the non-pathological psychosocial dimensions of refugees' lives that promote positive mental health" (p. 209). Attention to these everyday realities of wellbeing should also be taken into account by health geographers to better link notions of wellbeing to place.

\section{Cultural perspectives of wellbeing}

Kearns and Collins (2010, p. 20) have noted that understanding wellbeing through the lens of culture can serve as an interesting bridge between cultural and health 
geographies. There have been some works that have examined wellbeing through a cultural lens, such Panelli and Tipa's (2007) work on Māori wellbeing in Aotearoa New Zealand, arguing that a geographical lens on wellbeing needs to examine the place-based interconnections, between culture and the environment (cf. Panelli and Tipa, 2009). The authors outline four components of Māori wellbeing and health promotion (p. 453): i) Mauriora, a strong and secure cultural identity; ii) Waiora, healthy air, land and water that requires a balance between their use and protection; iii) Toiora, personal behaviours; and iv) Te Oranga, increased participation in the Māori community to contributes positively to one's wellbeing. However, what the authors found affected this ability for the Māori to obtain a sense of wellbeing was the inability for many to be physically connected to their ancestral lands, due to the loss of land through colonial treaties. Similar to conversations related to culture and health mentioned earlier, discussions of culture and wellbeing also reveal historical, colonial and political economic barriers some may face in accessing and feeling a sense of wellbeing.

Other works have highlighted links between migration and wellbeing, to show that "wellbeing is essentially grounded in spaces and places in which we live, work, and engage in social interactions" (Dossa, 2002, p. 341). This is a point also evident in Elliot and Gillie's (1998) work, which looked at wellbeing from the perspective of Indo-Fijian women in British Columbia. In this article, wellbeing was understood as referring to one's emotional health, such as daily stress, the divorce and geographic separation from a spouse, as well as stress from one's work. McMichael and Manderson (2004) also took migration into consideration in their work with Somali migrants in Australia, investigating the effects their social networks had on their notions of wellbeing. 
McMichael and Manderson write that "well-being is not just about contemporary social structures and activities, it is also affected by how women use the past to give meaning to the present" (p. 88). With this view of wellbeing that takes into account pre-migration memories of one's homeland, they detail their interviews with Somali migrants and notice a motif of viewing the country through an idyllic lens. Here, wellbeing connects with nostalgia and pre-migration memories in post-migration contexts.

\section{Therapeutic networks}

Smyth (2005) defines therapeutic networks as the "less formalized arrangements of support and care that often exist outside (or in parallel to) the traditions of biomedicine" (p. 492). Through therapeutic networks, everyday practices, like exercise, cooking and socializing with one's community members can all be considered healthpromoting practices. A therapeutic network approach is beneficial for understanding migrant health in everyday life because the idea of a network can illustrate how care and health is played out in a variety of places and scales, much like ideas proposed in a relational approach to health. As well, many of the works consider the role of transnationalism on health. As well, much of the literature using a therapeutic network approach typically focus on non-Western people, which is very applicable to my work with South Asians.

Menjívar's (2000) work illustrated therapeutic networks locally, through an examination of the social networks of Salvadoran immigrants in the United States. What is different about this piece is the attention given to untherapeutic networks of health, and Menjívar argues that "immigration scholarship is still concentrated on depicting a onedimensional view of immigrant informal networks that focuses almost exclusively on 
their positive aspects" (2000, p. 33). This stand against the portrayal of an idyllic migrant community was first brought forth by feminist scholars, who wanted to show broader, non-stereotypical images of immigrant communities (see Kibria, 1995; Mahler, 1995; and Woelz-Sterling et al. 2010). Much was explored in Menjívar's later piece (2002) on the local and transnational networks of healing for Guatemalan women in the United States. Chakrabarti (2010) explored the therapeutic networks of pregnant Bengali women in New York City, and pointed to long-distance calls to family in India as important in negotiating health practices in the diaspora. This work is important to my own research not only for its attention to South Asian communities but also how social networks are important in shaping and informing migrant women's daily health routines. Messias (2002) looked at a similar phenomenon among Brazilian women in the United States. Like Chakrabarti, Messias examined how families across borders share health information and practices but also took this further to consider how health practices can shape sense of self, as "the stories women told about transnational medication processes were as much about identity as they were about medicine" (2002, p. 188). From these networks, one woman began to see herself as being 'more Brazilian' because she began to rely on Brazilian medicine more while living in America, highlighting how therapeutic networks can connect culture and medicine, to produce a new sense of self.

Works done by medical anthropologists Thomas (2010) and Krause (2008) were also significant in theorizing therapeutic networks. Thomas recognized the role of travel between the UK, Zimbabwe and South Africa as an important feature of health care, as people travel to acquire traditional medicines from home. She calls the phenomenon of Zimbabwean and South African families sending medicines and medicinal knowledges 
across borders, transnational networks of health care. Krause's (2008) work coined the term transnational therapy networks, the "interlaced, situational, formal and informal contacts between people which have become meaningful in the event of sickness, providing financial and practical support" (2008, p. 235) for Ghanaians in London, UK. Both works can push health geography to look outwards to see how therapeutic networks can be multi-scalar. Escandell and Tapias (2010) explored how Bolivian immigrants in Spain use transnational therapeutic networks to access traditional medicine from Bolivia to treat emotional distress as a result of migration. This work is useful for revealing links between health practices and emotions, to show that emotions play a role in the formation and maintenance of therapeutic networks.

\section{Health and place: everyday health geographies}

The sphere and scale of the 'everyday' in geography has been overlooked, likely in the face and interest in works examining globalization and transnationalism (Dyck, 2005). Sociologist Henri Lefebvre is most well-known for his attention to the everyday in his work, Critique of Everyday Life, writing against ideas of the everyday as merely "'what is left over', after distinct, superior, specialized, structured activities have been singled out by analysis" (1991, p. 97). Instead, Lefebvre insists the everyday "has a secret life and a richness of its own" (p. 87). Connecting these assertions of the everyday to discussions of health and wellbeing can better reveal these micro-geographies of health. As such, the literature to be examined and reviewed here will speak to one or both points I have drawn from Lefebvre.

Some of the earliest works done by geographers that have combined everyday and health geographies have been through understanding disability, and conversations 
between disability studies and geography predate discussions of health. These scholars shifted from using a medical model, which focused on disabled people to be 'damaged', to a social model, which looked at interactions between people with disabilities and wider society, and how disabilities have or have not been accommodated. The another popular model was the environmental model, most discussed in geography, which refers to how everyday environments can crate barriers to people with disabilities, barring their participation in society. Geographers like Parr (1997a, 1997b, 1998a, 1998b,1999, 2000), Imrie (1996, 2000a, 2000b, 2004; Imrie and Kumar, 1998; Imrie and Edwards, 2007), Kitchin (1998, 2000, 2001; Kitchin and Law, 2001; Kitchin et al., 2001), and Chouinard (2000, 2012; Crooks and Chouinard, 2006) have been on the forefront of these debates and advancements of inquiry linking geography and disability.

Particularly, in these works, the body is an important geography for both disability and health. In the International Encyclopedia of Human Geography, Johnson (2009) wrote that "the body is a crucial site of socio-spatial relations, representation, and identities. It is the place, location, or site of the individual. It is also a site of pain, pleasure, and other emotions around which social definitions of wellness, illness, happiness, and health are constructed" (p. 326). In this definition, there is certainly room in considering the body in health geography when talking about health and illness. As well in the case of disability, the link between perceptions of disability and one's identity intersect in the space of the body. For example, Moss (1997) explored the material and social geographies of disability in the home, particularly among older women with rheumatoid arthritis. Moss was interested in a relational view of the home environment, beyond the fixed space of a house: 
relations that constitute the household are not spatially confined to the physical, material dwelling. They extend outward into automobiles, schools, community organizational meeting places, corner stores and other dwellings. Friendships and social networks also contribute to (re)structuring domestic space through providing emotional support and concrete assistance with maintenance tasks. (p. 24)

Taking into account these other spaces connected to the home through routines and movement is important when considering the sphere of the everyday.

Dyck's work from the early 1990s was also important in examining relationships between women's health, everyday life and work, discussing topics, such as chronic illness (1992), as well as migration experience (1995a). For instance, in one article (1995a), Dyck looked at the different pathways Chinese and South Asian women took to maintaining their health, one of the few works noting racialization and orientalism in health geography, applying an anti-racist, as well as a feminist lens. In another piece (1996), Dyck and Moss used a feminist political ecology framework to grapple with the roles the body and the environment play when discussing health in the everyday lives of women (also see Moss and Dyck, 1999; Moss and Dyck, 2003) Their understandings of health in everyday life was contextual: "by context, we mean the richly textured social formation wherein social formations are woven threads in a tapestry. Threads spun as interactions amongst people, are woven together temporally and spatially, imbuing our individual tapestries into a consecutive whole" (p. 739). Through the lens of the body, one is better able to understand the role of health, and how pain can interact with daily routines in the home, as well as in work environments. Taking into account these routines can show how chronic pain can be understood spatially. Additionally, using chronic pain to understand health and place demonstrates how places once associated with health can change through the nature of chronic pain, as it comes and goes unpredictably. With 
attention to place, the body, chronic pain and health, Dyck and Moss highlighted how these terms are all constructed and deconstructed through daily routines and movements.

Outside of linking disability, health, and place, there have been some research done by health geographers that have also touched upon everyday life. For instance, English et al. (2008) wrote an article detailing the everyday therapeutic landscapes of breast cancer survivors, and Liamputtong and Suwankhong (2015) explored much the same among Thai women, though included drawings as a way for participants to illustrate their perceptions of and relationships to breast cancer. Here, everyday places for health are in the body, demonstrating how embodiment may play a role in ideas of health. As well, these two pieces are relevant for their re-introduction of the body into discussions of both everyday health and therapeutic landscapes in contemporary literature. Wendt and Gone (2012) looked at the everyday landscapes of health of urban Aboriginal people in the Midwestern United States, noting the significance of an urban indigenous health organization. Others, like Kearns and Wilton have sought to use health geography to better understand geographies of HIV/AIDS (Kearns, 1996; Wilton, 1996, 1999). In particular, Thomas (2007) used a diary method as a means to better understand the everyday geographies of HIV/AIDS in South Africa, considering how the disease features daily life without the gaze of the researcher, with participants noting their experiences in a solicited diary. Thomas also looked at ideas of wellbeing to demonstrate how both HIV/AIDS as well as emotions were sources of health knowledge, as well as how these are culturally-based (pp. 75, 76). 


\section{Everyday health practices}

There have been some works that have examined how health is practiced, as well as spatialized. Thrift (1999) explains that "it is not always enough just to 'be' in a place to guarantee a 'therapeutic' outcome: rather there is a need for a skill or artistry in our engagements with place" (pp. 310-11). Attention to health practices over health spaces or landscapes moves the focus to how people can create places they associate with health and wellbeing in their everyday lives. To this, Dunkley (2009) coined the term therapeutic taskscapes, an offshoot of anthropologist Tim Ingold's notion of the taskscape $^{4}$, to understand how practices at a camp for troubled youth can be considered therapeutic. Therapeutic taskscapes are useful in examining the role of everyday practices because they "identify key health-giving activities in order to theorize how well-being is produced from relations and activities" (p. 95). Dunkley's work shows that notions of wellbeing, as well as health can be better understood if researchers paid more attention to how practices play a role in place-making.

Much like Dunkley's attention to routines as core in place-making, this same point can be extended to better understanding migration, through the concept of transnational habitus. Borrowed from Bourdieu's notion of the habitus ${ }^{5}$, Guarnizo (1997, p. 311) defines the transnational habitus as

a particular set of dualistic dispositions that inclines migrants to act and react to specific situations in a matter that can be, but not always, calculated, and that is not simply a question of conscious acceptance of specific behavioural or sociocultural rules. The transnational habitus results from the migration process itself, which has spread people's lives across national borders and becomes like a

\footnotetext{
4 "an array of related tasks" (1993, p. 1524). Ingold was interested in how everyday tasks can be a lens to better understand the production and significance of landscapes.

${ }^{5}$ Bourdieu (1990) was interested in structural influences, as well as glimpses of agency and tacit knowledge to account for everyday practices.
} 
second nature. The transnational habitus incorporates the social position of the migrant and the context in which transmigration occurs.

Looking at transnational habitus can explain why migrants employ particular practices while in the diaspora, showing their ties to routines back home, as well as highlighting how migration can be better understood through daily practices. Gastaldo et al. (2004) pushed for greater attention to ideas of place and wellbeing in their work. This piece is worth noting, due to its keen attention to how recent migrants create mental healing landscapes in their everyday lives. Gastaldo et al. use the term therapeutic landscapes of the mind to refer to the "personalized, dispersed, of everyday use, and mediated by individual experience of places [...] they are constituted by each individual through a web of emotion and social relations that occur in relation to a given place" (pp. 169, 170). The migration experience differs from person to person so it is important to acknowledge different modes for coping, such as making photo collages, trying different foods, travelling through airports, etc.

Literature in health geography and public health converge when discussing the practice of walking to maintain health (Gatrell, 2011, 2013; Doughty, 2013; Andrews et al., 2012; Ettema and Smajic, 2015). One of the major arguments among health geographers appears to be that more attention needs to be paid to the political economy that informs people's decisions to walk, such as not having access to a vehicle (Bostock, 2001). Public health researchers on the other hand, should begin to consider how walking may be both a health routine but also associated with emotional aspects of wellbeing, a practice which can be emotionally satisfying. Andrews et al. (2012) speak to this gap in health geography, and point to some exclusionary elements in walkability research, noting an absence of researching with fat bodies (p. 1927), disabled bodies (p. 1928), as 
well as walking for reasons outside of maintaining one's health, such as protests and movements, as well as spirituality and religious purposes (p. 1930). Likewise, Gatrell (2013) used the term therapeutic mobilities to make links between health and place, through walking. Attention was paid to how daily movement, as well as acts of migration are both important aspects of mobility that can be encapsulated through this term. Gatrell goes into greater detail by looking at the act of walking alone, with a group, and the role of the natural environment and surrounding neighbourhoods in influencing or affecting how therapeutic walking can be for individuals. Gatrell feels that health and wellbeing should not be confined to landscapes, and more attention should be given to the act of moving as therapeutic in its own right. In all, looking at how wellbeing and health are understood in daily life can better reveal the subjective 'therapeutic' quality of places and the practices behind them.

\section{Geographies of care and caring}

In many respects, health geographies and the geographies of care share similarities. For one, the two sub-fields began with an interest in broader understandings of care or health (Parr, 2003). Specifically, care geographies have moved from their topdown analysis of the place effects of state welfare regimes (Staeheli and Brown, 2003), to how care functions in everyday lives. Additionally, some works connect back to health geography through the application of ideas of home and therapeutic landscapes as a way

to understand care (Dyck et al., 2005; Williams, 2002; Milligan et al., 2004; Milligan and Wiles, 2010). Much like my interest in using a relational approach to better understand health, Milligan et al. (2007) explain that "care has been interpreted within a relational 
framework that examines health, care-giving and the receipt of care in relation to the places in which they occur" (p. 135). Some works have used care geographies as a lens to explore relations between the ethics of care and policy (Parr, 2003; Popke, 2006; Lawson, 2009; McEwan and Goodman, 2010), and others have noted debates on the responsibility of the state in terms of care (Barnett, et al. 2005; Smith, 2005). Clearly, care has many different connotations in the discipline. In an editorial, Atkinson et al. (2011) defined care as "concept, emotion, practice, politics [and] moral exhortation" (p. 563). For my own research, the particular stream of care geographies research most relevant is that which considers care as a practice (Milligan et al., 2007), as well as care as an emotion.

\section{Care as practice}

In an editorial concerning care geographies, Conradson (2003) highlighted that practices of care "are implicated in the production of particular social spaces" (p. 451). His definition of care is not only limited to being found in practices but also through emotion, with reference to Silk (2000), who saw care as the proactive interest of one person in the well-being of another (cf. Conradson, 2003, p. 451). By examining particular practices and places, different ideas of care can be revealed. In another article, Conradson (2005) discussed how care can be understood relationally for older adults in the UK, in the space of a care center. Through noting participants' perceptions of the space, as well their experience of care in the center, Conradson found that many enjoyed their stay there for four reasons: i) distance from daily routines; ii) interactions with nature; iii) the development of new friendships; iv) as well as new dimensions of selfhood (p. 342). What is interesting about this piece is the attention to how practices of care can influence how individuals interact with their surrounding landscape, a process 
called the self-landscape encounter, "the interactions between individual and landscape [that] are understood as being complex and multifaceted, emerging out of particular embodied encounters but also subject to later interpretation" (p. 338). This last part of the definition is important, demonstrating how experiences of care and place can change over time. This point is important because I want to take into account how ideas of health and wellbeing possibly change through experiencing migration. Clearly, care, much like health, is placed and temporal, as well as dependant on contexts, like migration.

Another important insight emerging out of this body of literature is the value of viewing these practices not from the view of those receiving care but those giving care. Wiles (2003) feels attention to the daily practices of care givers should be seen as relevant in care geographies, as the point of view of the caregiver can bring forth new care geographies to be explored. Wiles noticed that "public and private areas of the home, like the bedroom and washroom become blurred through the act of caring. Furthermore, examining these practices and challenges through the lens of emotional geographies may also be relevant here. Wiles explained that "caregivers feel responsible for a range of things from personal safety to bowels to appetite and food intake and medications" (p. 1315). As such, the emotional and place-making properties as a result of caregiving are equally relevant to investigating health through the lens of the everyday life, primarily in the home.

\section{Caring: the emotional component of care}

The second stream of care geographies literature examines care as an emotion. This body of care geographies is interested in how the act of caring can be read as a form 
of emotional labour ${ }^{6}$, a concept borrowed from sociology. Milligan explains that this literature also grew from looking at care from a gendered lens which saw women being the primary agents of caregiving. Caring, or the emotional interactions of care are what Milligan (2005) calls "a double-edged sword" (p. 2108): on the one hand, "they can bring feelings of satisfaction and immense reward to what is often a frustrating and difficult job. On the other, it exposes care workers to the feelings of sadness and loss" (p. 2108). Highlighting both the intrinsic and more difficult emotional encounters can show the many layers of care geographies.

Evans and Thomas (2009) investigated the emotional encounters when considering caregiving and HIV/AIDS. Much like Milligan (2005), Evans and Thomas found that these close, intimate relationships between caregivers and the cared in the familial context can lead to many unspoken, repressed emotions which can affect the quality of care given. Their work is in the context of those afflicted with HIV/AIDS in Tanzania, Namibia, as well as in the UK, noticing that cultural norms are important to consider (Evans, 2010). For example, Evans and Thomas (2009) noted the importance of children in taking care of their parents and elders as an "intergenerational contract" (p. 114). In some cases, the emotions of the caregiver had to be put to the side for the sake of the person being cared for. Their work is useful for taking into account how some manage HIV/AIDS daily, with great attention the emotional dimension of both those with the disease, and those caring for them.

\footnotetext{
6 The management of one's feelings while in the workplace, and the effort or labour in which this task requires (Hochschild, 1983)
} 


\section{Everyday geographies of immigrant and refugee health}

Attention to immigrant and refugee health is slowly growing in health geography. Ho and Hatfield (2011) insist that to truly understand migration is to place it within the everyday lives of migrants, as "the notion of the everyday offers a way of getting beyond generalised understandings of globalisation and transnationalism towards recognition of the ordinary, daily reality of what it is to live in and across different yet specific places and spaces" (p. 710). In their article, they propose that geographers should study migration through sociality and materiality, with sociality referring to the social lives of migrants, and materiality referring to the "material cultures that constitute the social and cultural worlds in which we live" (p. 709). Taking note of both sociality and materiality through works done by geographers and other social scientists can reveal how migration, like health and wellbeing, can be understood the lens of daily life. To this, the literature cited below all exhibit some elements of sociality and materiality.

Cattell et al. (2008) noted relationships between public space and wellbeing in a multi-ethnic London, UK. In this article, the importance of everyday spaces, like parks, and ethnic stores is highlighted. In reference to policy in the UK, Cattell et al. insist that greater attention needs to be paid to these spaces, and their potential in fostering both a sense of wellbeing and community. Ortega-Alcázar and Dyck (2011) also looked at public space through photographs taken by participants. They found that examining these photos revealed similarities between peoples of colour and Westerners, like the visiting of parks and grocery shopping. However, these photos also showed differences, such as references to traditional remedies, like the consumption of pepper soup by Nigerian immigrants (p. 117). Through the photos, the authors portrayed participants as agents of 
health knowledges, against public health assumptions of recent migrants as not knowledgeable about managing health.

Dyck has been a prominent figure in examining immigrant and refugee women's health, primarily in Vancouver, British Columbia. In addition, Dyck's contribution to the publication of the edited book, Geographies of Women's Health (Dyck et al., 2001) was another major step to conceptualize the diversity with which women experience and understand health (cf. Williams and Garvin, 2004), with particular emphasis on nonWestern women's notions of health. One of Dyck's earliest pieces pushed health practitioners to consider the role of culture when treating immigrant clients (Dyck, 1989) in the field of Occupational Therapy. Dyck's later works in the mid-1990s took these ideas in health geography, and were keen on linking ideas of health, place and work among newly arrived groups. For example, in another piece (1995b), Dyck looked at the understandings and everyday maintenance of chronic illness among Chinese and South Asian women in Vancouver. One of the most important points noted was the need for geographers to consider the roles of culture but also discussions of race when considering these women's health maintenance routes, as Dyck felt ethnic sensitive and anti-racist model used by health providers and researchers fail to take into account "the fluidity of culture and ethnicity, and of the social construction of race" (p. 248). In other words, there is little consideration for how culture changes or how race is not merely a biological construction but one that takes place within everyday assumptions or prejudice and interactions. In terms of the results, Dyck (1995b) found that most Chinese and South Asian women did use non-biomedical resources to maintain their health, and nearly half used these along with treatment given to them by doctors (p. 251). As well, many of the 
women relied on their own local social support networks in the community for health advice (p. 254). These findings demonstrate the complexity in women's decisions for choosing particular remedy routes. For many, it is a matter of keeping cultural ties, while for some, there were socio-economic underpinnings (pp. 252, 253). Here, culture, place and income all contribute to how health is both understood and managed by these women.

Nearly a decade later, Dyck (2006) looked at the health practices of South Asian women in Vancouver. In this work, the everyday was important, as "the spaces of quotidian life are sites of cultural reproduction, contestation and transformation, with active sorting through health and healing knowledges" (p. 3). Here, Dyck clearly saw narratives as health knowledges encapsulated in everyday places, always undergoing change. In particular, social interactions and preparation of South Asian foods and remedies, like $\operatorname{samf} f^{7}$, were seen as health practices but also cultural ones, to keep a 'remembered India' intact. To this, Dyck provided discussions that demonstrated how these knowledges interact with so-called Canadian routines, like visiting a doctor before trying cultural health remedies, or the eating of fast food over ethnic foods (pp. 10,11). In this article, what constitutes 'place' is twofold: those places experienced through the body, as well as those places re-crafted and negotiated as a result of these daily health practices.

In the following year, Dyck and medical anthropologist Dossa (2007) investigated the everyday health practices of Sikh and Afghan women in Vancouver. This work was insightful for distinctions made between these two groups of women. For example, the Sikh women mainly came to Canada as family immigrants, while many of the Afghan

\footnotetext{
${ }^{7}$ fennel
} 
women came as refugees. As well, many of the Sikh women had not worked for pay in India but worked in Canada. The Afghan women in contrast, experienced de-skilling, having either or both high school and university education, as well as previously working in laboratories, working as teachers, or seamstresses prior to moving to Vancouver (p. 694). Many of the Afghan women headed single-parent households, losing family and partners to the war, in contrast to many of the Sikh women married to men who came to the country as economic immigrants (p. 695). These varying social positions are important to highlight, as they can make a difference in terms of access to the health care system, as well as access to local ethnic communities for health advice. There are several key points emphasized in this article. One, that educational levels do not map neatly onto the numbers of women who use traditional remedies versus those who use biomedicine (p. 697). To this, Dyck and Dossa explained that it is their experiences in migration and their constant remembering and recalling of home which push many to negotiate traditional and biomedical health practices in the spaces of their homes. Second, that the use of traditional medicine should not be seen as inferior to biomedicine. Finally, that the concept of therapeutic landscapes can be used to better understand the everyday healthy places created through daily routines, like child rearing, cooking, working, religious observance, etc. This flexible application of therapeutic landscapes in the literature is limited (Dunkley, 2009 is an exception) and certainly should continue to highlight these micro-geographies of health.

Outside of geography, there are researchers that have paid attention to ideas of wellbeing in everyday life in the diaspora, like anthropologists Dennis and Warin (2007) and oral historian Palmer (2012). Dennis and Warin (2007) examined how Persian 
women in Australia use cooking as well as sewing and stitching as ways to remember a former Persia before to the Iranian Revolution of 1979. Through the body and the scents of desserts they make, home is constructed temporally and remembered, contributing to a sense of wellbeing for the women. Through the medium of food, Palmer (2012)

illustrated how the Ethiopian buna $^{8}$ ceremony is used to maintain one's cultural identity, as well as establish therapeutic social ties to other Ethiopians in London, UK (p. 68).

Palmer framed his work through ideas of health and place, with interest in these everyday ethnic spaces, like community centres, homes or coffee shops and their therapeutic potential, calling scholars to not overlook their importance for health and place in ethnic communities (p. 74). Works such as these outside of health geography demonstrate how wide-reaching and influential the discipline has become, and shows how important the relationships between health, place and wellbeing are in migration and health research.

\section{Conclusions}

This chapter began with a brief introduction to some debates in medical and health geography surrounding ideas of place. Most theorists seemed to agree that place is related to one's lived experience in particular environments, and both health and wellbeing can be also be considered in particular lived experiences. I then moved into discussing the importance of a relational approach to health and place, through Cummins et al. (2007). In their work, it was explained that to better understand health, more expansive and holistic approaches to place need to be taken. This relational approach understood place in a network approach, influenced by a variety of different circumstances, environments and actors. For my work, this relational approach considers

\footnotetext{
${ }^{8}$ coffee
} 
the role of migration in shaping people's conceptions of health in place. From place, I moved into the review of the concepts used in this study: health, wellbeing, and therapeutic networks, highlighting the literature most relevant to my own research. With health, attention to balance, as well as culture, religion and spirituality were important. As well, I noted mainly geographical works that discussed emotional, economic, and cultural associations of wellbeing. Finally, with therapeutic networks, I discussed works that highlighted a network and multi-scalar approach to the term.

My attention to reviewing the literature most relevant to this research was ultimately interdisciplinary: I began with a call to introduce Lefebvre's (1991) notions of the everyday as a way to better situate health in this context. To do so, I divided the review into three main bodies of literature: i) everyday geographies of disability; ii) care geographies; and iii) everyday geographies of immigrant and refugee health. Works from disability scholars in geography, like Moss and Dyck are particularly notable in their attention to how their lived experiences of physical disability and chronic pain mediate daily routines, and in turn, create everyday domestic geographies. Care geographies were useful in the attention to how care as a practice and as emotion influence everyday health geographies. Like health geography, care geographies have too narrowed their focus on the micro-geographies of care, examining links between notions of care, the body and place, and Conradson $(2003,2005)$ has been particularly insightful for making these links more salient. For immigrant and refugee geographies, I noted Ho and Hartfield's (2011) assertion that these everyday geographies should pay attention to how migration is woven through, both socially and materially in daily life. Authors, like Catell et al. (2008) and Dyck (2006; Dyck and Dossa, 2007) have brought greater attention to elements of 
sociality and materiality in regards to health in this literature. In all, through an application of relationality, I hoped to expose some of the ways in which health and wellbeing reveal themselves in myriad ways in everyday life. The following chapter will outline the methodologies and methods used in this research. 


\title{
Chapter 3: Methodologies and research methods
}

Everyone who writes about the Orient must locate himself vis-a-vis the Orient.

\author{
- Edward Said, Orientalism
}

\section{Introduction}

The introduction to this thesis highlighted my desire to use a feminist methodological framework, which is concerned with portraying participants in their own voices. In this chapter, I unpack these methodologies and highlight how I have met them throughout the research process, from structuring the research, to data collection, then to analysis and reflection. To demonstrate these points, I split this chapter into five sections. First, I outline the concepts important to a feminist methodological framework, namely positionality, power, subjectivity, emotion and reflexivity. Second, I will justify and explain my decision to use qualitative methods, specifically semi-structured interviews for my research. Third, I will reflect on my research experience, through unpacking conversations surrounding reflexivity in qualitative research. Then, I will turn to breaking down the stages of my research 'failure', and discussing many of the obstacles I faced as a researcher. Fourth, I speak at length on some of the ethical concerns of this research, and how I have tried to address them to minimize risks for participants. Lastly, I explain my method of analysis and interpretation of the research data. Overall, the goal of this chapter is to show how this research was carried out.

\section{Feminist methodologies in health geography research}

The geographies of health explored in this project will be brought out in the use of feminist methodologies. Feminist methodologies provide an "analyses of the 
complexities of power, privilege, oppression and representation" (England, 2006, p. 286). My application of feminist methodologies is used to highlight informants' everyday experiences and understandings of health. It is important to view these narratives as 'placed', useful to this research "to demonstrate the productive capacity of everyday routines in forging healthy space within the particularities of migrant settlement" (Dyck and Dossa, 2007, p. 691). I now turn to important dimensions of feminist methodologies in this research: postionality, power, subjectivity, emotions, and reflexivity in research.

\section{Positionality}

Attention to my positionality as a researcher is relevant. England defines positionality as "how people view the world from different embodied locations" (2006, p. 289). Positionality is especially important to consider because it has the ability to shape research outcomes, and what becomes considered valuable data in the research. These outcomes are also shaped by the interactions between researcher and participants, and both these components of positionality will be expanded upon below. Although interacting with participants was limited due to time and scheduling, I still feel that my positionality was greatly influenced through these interactions via the semi-structured interviews that I conducted. Through two gatekeepers in the Newcomer Clinic, I was able to gain entry into the Nepalese and Bhutanese community in Ottawa. The Ottawa Newcomer Clinic works to assist newly arrived, government-assisted refugees, and this assistance is mainly related to health but also social and economic needs. The group that was affiliated with the Clinic were the Bhutanese informants I spoke to, a group recommended to me by one of the gatekeepers, Siffan. Siffan and I spoke throughout the summer to help me to gain approval from the Clinic's Human Resources, as well as from 
the Somerset West Community Health Centre (SWCHC), which the Newcomer Clinic is affiliated with. Siffan was very sympathetic to the pressures to move the approval process along, being a Masters student herself in the past. This mutual understanding could be argued as a way of making me an 'insider' through Siffan, and our shared educational background. In the fall, I was introduced to Abha, a health navigator with the Clinic. She was recommended to me by Siffan, with her connections to the broader South Asian community in general but specifically with the Nepalese population which she is a part of, and the Bhutanese clients she works with. Like Siffan, Abha was very considerate of my timelines, as well as having a keen interest in the project, as she sees the issues in accessing health among the Bhutanese population, first hand. Abha provided a lot of ideas and direction for my project, as well as solely handling interpretation during interviews.

I note these two key gatekeepers in this section to highlight how positionality can be understood in a number of ways. For me, I felt that education, and likely age, aided me in gaining trust and access to the informants I would later interview. However, positioning myself as an academic researcher may also affect my relationship with participants and create hierarchies. England reminds us that "we cannot fully locate ourselves within our research because we never fully understand our position in webs of power" (1994, p. 289), as our knowledge as researchers is only partial, and these partial knowledges are called situated knowledges, knowledges connected to and constructed by our position(s) in the world (Rose, 1997). Here, it is worth highlighting the significance of Edward Said's quote placed at the start of this chapter. Said believes that to write about the so-called 'Orient', which is now consists of the continents of Asia, Africa, Latin 
America and Australia, we as writers need consider our place in the world. Here, my position and identity as a person of colour are noteworthy. I am a Canadian-born Muslim Somali woman with immigrant parents. I self-identify with these three identity markers of religion, ethnicity and gender but also see myself as a racialized person. Although I felt that my ethnicity, race and religion did not play an evident role as a boundary maker, I still feel these parts of me are still worth noting. Admittedly, one of the assumptions I had carried into this research was that my informants would be able to openly speak and share with me, as a fellow person of colour. I had also assumed that because I am a person of colour with immigrant family, I would understand some of the social and economic issues facing many of my informants. Yet, from the literature, I felt many of these assumptions were not taken into account when doing research. Some geographers think this may be the result of a lack of people of colour as prominent geographers (Kobayashi, 1994), though I would imagine this to be changing. One of the major misgivings I have as a researcher of colour is the possibility of blurring the narratives of my informants, as if all Bhutanese and Nepalese people are facing the same issues. Kobayashi cautions geographers to consider their place in their research, stating: "I do not use other people's struggles as the basis for my research; I use my research as the basis of struggles I am a part of" (1994, p. 78). In other words, a constant self-reminder is needed to see these narratives, stories and memories as contextual and situated, not essentialized; each informant has a unique story to share. With this methodological lens, awareness of participants and of myself as a researcher and individual can be taken into account.

Positioning oneself distantly from participants can also be problematic. For 
example, Mullings (1999) argues we need to move beyond the insider/outsider dichotomy and examine how positionality is far from stagnant in research. Mullings insists that geographers pay attention to positional spaces, the "areas which situated knowledges of both parties of the interview encounter, engender a level of trust and cooperation. These positional spaces are often transitory and cannot be reduced to the familiar boundaries of insider/outsider privilege based on the visible attributes, such as race, gender, ethnicity or class" (p. 340). I feel a turn to positional spaces would likely better serve my research because it can account for my changing of positionality, with different participants, in different places, at different times.

\section{Power}

A difficult issue to overcome in qualitative research is power relations. Power, however, can be negotiated and diffused under certain conditions. Therefore, much like positionality, acknowledging power relations in research in specific sites is also an important component of feminist research. Winchester (1996) writes that "power relations influence both the access to target groups and the structure and conduct of the interview" (p. 122). This research, like many research projects, also had power at play. For instance, recruitment through the Newcomer Clinic can be considered as an issue of power, as a suggestion to contribute to a research project as a participant can be felt as a sense of obligation than out of pure interest. (Karnieli-Miller et al., 2009, p. 281). Another example of unequal power relations in the research was with developing the structure of the project. With help from my research supervisors, the questions, aims, objectives and methods were my choosing. I did not consult informants about how to structure this project, which meant I held a great deal of power in which direction the 
research headed, even before interviews were conducted. A point to consider here is my aims as a researcher, and these connections to possible power structures:

Relationships are affected by the content of the inquiry, and equally, by the institutional context in which the study is carried, and by the researchers' and participants' personal motivations. These motivations can be complimentary or contradictory, overt or covert. Oftentimes, the researcher's motivations to initiate research projects include fulfilling their professional interests, such as publishing, carrying an academic degree, or receiving funding. (Karnieli-Miller et al., p. 281)

Research aims, then, can be both theoretical and material. For one, I came to do my Masters degree to aid in my chances in finding long-term employment, and to possibly publish articles on my work. The theoretical benefits come from working in the relatively new sub-field of health geography. Through my work, I was hoping to be a contributor, to push more for attention to perceptions of health in everyday life, and see health as a form of knowledge among newcomer populations. This last point was on the back of my mind, which consciously and unconsciously shaped the structure of this research, including research questions and objectives.

Power relations may also shift during the research process, as participants, too, demonstrate degrees of agency. One example is to note the material benefits to be received by participants. My Letter of Information and Consent Form promised those interested that I would provide participants with one to two sheets of bus tickets after the completion of the interviews. This was an idea suggested by Abha, who felt that this would be valuable to the Bhutanese informants I would speak to, due to the high cost of public transit. Also, I had to consider how interviews might have been seen as a good outlet for participants to mention their concerns with their health and health navigation in Ottawa. These two examples could have motivated some to participate, which could be seen as a shift in power. 


\section{Subjectivity}

Positioning oneself as beyond a so-called objective, non-feeling, unbiased researcher is an important dimension of feminist qualitative research, and subjectivity allows these factors to be explored. To Dowling (2010, p. 35), "subjectivity involves the insertion of personal opinions and characteristics into research practice". In this light, subjectivity is similar to positionality but also accounts for the emotional outcomes of research and how this has affected the researcher, and vice-versa. Yet, with so much attention being paid to positionality, there has been little said about how one's personality can be a factor that affects research findings, as well as building rapport with participants. Moser (2008) believes personality to be the 'new positionality'. Through her fieldwork in Indonesia, Moser insists her positionality as a First World, White Canadian researcher did not bar her from fully interacting with locals. Instead, Moser felt it was her personality that helped build rapport with participants:

Where the focus is on how we are positioned vis-à-vis our research subjects with regards to race, religion, class, etc, there is a silence regarding how we as individual researchers behave and act with research subjects who also have a range of social skills and emotional abilities. The individual's personality places a significant role in shaping power relations in all social encounters, including that of the researcher and researched. (p. 386)

In my research, I wanted to come off not as an overbearing researcher but one who was respectful of informants' stories, as well as be appreciative for their participation. In terms of my positionality as a person of colour, I felt did not matter to the participants I was speaking to, as they were very kind and open to sharing. I felt this patience showed, as I encouraged participants to take the responses in terms of my research questions in whichever direction they liked. Looking back, had I gave more firm directions, I would not have received the data I did, as participants linked health and wellbeing to many 
different parts and practices in their everyday lives. However, one of the limitations I had was the inability to demonstrate intersubjectvity, which "refers to the meanings and interpretations of the world created, confirmed, or disconfirmed as a result of interactions (language and action) with other people in specific contexts" (Dowling, 2010, p. 35). Because several of the Bhutanese informants did not speak fluent English, this relationship remained purely research-based. I felt there was little opportunity to engage some participants because of this language barrier.

\section{Emotions in qualitative research}

Feminist researchers were among the first to pay attention to the role of emotions in research (Bennett, 2004; Holland, 2007; Hopkins, 2007; Watts, 2008; Dickson-Swift et al., 2009; Evans, 2012; Jones and Ficklin, 2012; Carroll, 2013; Bondi, 2014), and much the same can be said about feminist researchers in geography. Currently, this line of inquiry has resurged, with geographers bringing light to this in the sub-field of emotional geographies. Attention to the way emotions can colour one's position as a researcher, as well as viewing emotions as a form of knowledge, are also both important considerations in qualitative research. Widdowfield (2000) notes an absence of emotions in academic work, highlighting that "unless or until discussing the way in which emotions impact the research process, and gain widespread credibility within academic debate, there is a risk that such discussion will have an undermining effect with either the researcher and/or the research itself being dismissed as 'too emotional'" (p. 201). This unspoken fear of being seen as too emotional appears to scare researchers in sharing research struggles. Widdowfield feels that an open academic environment can provide a safe space to discuss these emotional experiences (p. 201). 
Despite this positive view of emotions in research, Widdowfield notes that there are still drawbacks to consider. For one, "it can be considerably difficult to put these emotions in words" (p. 201). Second, there can be a question of knowledge lost in translation that should be considered. Since research and thesis writing can be long processes, many of these emotional experiences, then, may be seen as minor issues, and no longer seen as frustrating or traumatic at a later time. These points only highlight the importance in recognizing how research is a process that can change our understanding of ourselves as researchers. Another drawback Widdowfield mentions is the "danger of indulging in a level of self-absorption which privileges the voice of the researcher(s) over that of the voice of the researched" (2000, p. 202), which was a major concern for me. I felt as though revealing these obstacles could take away from the rich data generated from my interviews with participants. Geographers like Bondi (2009) feel that attention to emotions in research is important for a researcher because it can demonstrate how the research has affected them, which in turn can better highlight one's sense of reflexivity, a term called emotional knowing. Emotional knowing "refers to situated ways of understanding our worlds, in which the subjective positions and contexts of all those involved in knowledge production (researchers and research participants) are acknowledged and viewed as integral to processes of knowing" (p. 449). Emotional knowing, then, is three-fold: i) it is used as a way for the researcher to comment on their feelings related to their work; ii) accounts for the feelings of participants; and iii) how emotions are knowledges in themselves, a connection to situated knowledges. My desire to highlight the importance of emotions in qualitative research relates to my own research struggles: the delays in getting field work started in my research, the frustration of 
waiting, and later the issues with thesis draft writing, and self-esteem as a graduate student researcher more generally. Some of these feelings were recorded in a notebook I kept during the duration of the summer and fall of 2014. Another dimension of emotional knowing is co-construction, which refers to the sharing of these emotions, is also important to note. I occasionally shared my frustrations with some fellow graduate students, my supervisors, and family. I also had the opportunity to share my struggles with first year Masters students in my department, which turned out to be a therapeutic experience. In the next section, I will go into more detail about emotional research and provide some entries from my field journal, connecting these to discussions on reflexivity in research.

\section{Reflexivity: rejoice, rejection and redemption of research}

As mentioned on several occasions, one of the cores of feminist research is the attention to our positions as researchers: how this may affect our perception and analysis of research data, as well as our relationships with participants. In this section, I address one of my objectives: to acknowledge the struggles I had in completing this research. Because of the twists and turns this project had taken, I felt it best to discuss these challenges at length, as a means to illustrate what reflexivity means to me. I begin with some definitions, conceptions, and debates surrounding reflexivity by other geographers and social scientists. I then turn to my own rendering of reflexivity by highlighting different layers, which I name rejoice, rejection, and redemption, each corresponding to a stage in the research process. 


\section{Reflexivity: some definitions and debates}

According to The Dictionary of Human Geography, "reflexivity entails consideration of a variety of factors: personal biography, social situation, political values, situation within the academic labour structure, personal relationship to research respondents, relations of authority within the research process" (Gregory, et al., 2009, p. 627). To these geographers, reflexivity is an accumulation of positionality, subjectivity and justification for one's research aims but not all see reflexivity in such a neat way. For example, Moss (1995) believes reflexivity should be understood both internally and outwardly. Moss defines reflexivity as "those introspective aspects of thoughts that are self-critical and self-consciously analytical (p. 445). This process of exploring one's position in their own research is also echoed by England (1994) understands reflexivity as "the careful considerations of the consequences of the interactions of those being investigated. And the reflexive 'I' of the researcher dismisses the observational distance of neopositivism and subverts the idea of the observer as impersonal machine" (p. 82). Although this is an interesting way of understanding reflexivity, it seems that England is not clear on what she means by this process of 'self-discovery', or what or who 'I' is. What role do participants and positionality play in researcher reflexivity?

In contrast, Rose (1997) advocates for a reflexivity of uncertainty that exposes difference. Rose writes that reflexivity should become "less a process of self-discovery than of self-construction" (p. 313), and is skeptical of a so-called transparent reflexivity: "the imperative of transparent reflexivity assumes that messiness can be fully understood" (p. 314). To Rose, some feminist geographers assume reflexivity would be able to shed light on all aspects of our research performance, such as better understanding 
our positionality and situating our knowledges. Another point Rose addresses is that "the failure of transparent reflexivity [...] does not indicate the failure of the project to situate knowledge reflexively" (pp. 314-315). I understood this point to mean that transparent reflexivity tries so hard to be transparent that it essentially misses its function: to be reflexive. To even consider reflexivity, Rose suggests that the researcher must consider their positionality, their standing or footing in the research to begin to move forward. In other words, self-construction needs to come before self-reflection.

Others like Pillow (2003) and Kobayashi (2004) have been quite vocal about their concern with how reflexivity has been applied. Pillow for instance, views reflexivity as being difficult to conceptualize because there is no uniform understanding, and more broadly, believes reflexivity has almost become shallow and diluted, with countless personal accounts of researchers in the social sciences. Pillow (2003) feels accounts of reflexivity should strive to "acknowledge the researcher's role(s) in the construction of the research problem, the research setting, and research findings [...] In this way, the problematics of doing fieldwork and representation are no longer viewed as incidental but can become an object of study" (p. 179). Considering reflexivity as an object of study rather than a hindrance is important to me, precisely the reason why I discuss it at length.

Like Pillow, Kobayashi (2004) insists self-reflexivity needs to be better conceptualized in the discipline, writing that "reflexivity opens us to the charge not only that it is a selfish, self-centred act that is the very antithesis of activism, but that it can even work actively to construct a sense of the other, to deny the reflexivity of others, and to emphasize the condition of alterity" (p. 348). To Kobayashi, reflexivity can produce just another stage for othering to occur, by privileging one's own thoughts or worldviews, 
and writing against the situated knowledges of others. Kobayashi (p. 348) explains that for reflexivity to be effective and critical, researchers should keep a few points in mind:

First, I think we need to think carefully about the limits of reflexivity, acknowledge the importance of it as an ancillary rather than the central part of our research [.... Second, we might think about the extent to which our more reflexive moments need to be shared with the world in order to make our points [...] Third, reflexivity has no meaning if it is not connected to a larger agenda-for which most of us avowedly both political and personal meant to change the world. How we choose the change the world is very personal, but the results are not.

Kobayashi's recommendations are very applicable to my own understandings of reflexivity. For one, I do not plan to make reflexivity the main theme of my research, nor did I go into the field trying to be 'reflexive'. Instead, coming across challenges helped my look inwardly into what reflexivity came to mean to me as a researcher. Secondly, I was very hesitant about sharing these experiences and even detailing them in this thesis (this was something suggested and encouraged by both of my supervisors). Lastly, my personal agenda is to not only reveal the emotional terrain of qualitative health research but also as a way for other young researchers to see these struggles are not shameful but very common. In terms of my political agenda, I use this section as a way to speak to the lived geographies of health research in geography.

Moreover, Pillow argues reflexivity in the literature has been discussed in four ways: "reflexivity as recognition of self, reflexivity as recognition of others, reflexivity as truth, and reflexivity as transcendence" (p. 175). Like Pillow, I felt the best way to reflect my understanding of reflexivity was to divide it into four sections, each reflecting a certain stage of the research, which I named rejoice, rejection, redemption, and postreflection ${ }^{9}$. A major point I took away from Pillow's work was to truly account for

\footnotetext{
${ }^{9}$ the fourth layer, post-reflection, will be discussed in Chapter 5
} 
moments of uncomfortable reflexivity: "the qualitative research arena could benefit from more 'messy' examples, examples that may not be as successful, examples that do not seek a comfortable, transcendent end-point but leave us in the comfortable realities of doing engaged, qualitative research" (p. 193). This is what I hope this sub-section will do: illustrating some of the 'messiness' I encountered, own up to some of my less successful moments in completing my Masters Thesis. To illustrate these challenges, I will place a table documenting these many research recruitment stages, broken down by month, year and method of correspondence at the end of this chapter. I now turn to my personal palimpsest of reflexivity.

\section{i. $\quad$ Rejoice: initial success \\ [ri-jois]: to be glad, take delight}

Many researchers typically struggle in the early stages of research; my experience was very different. In my second semester of my first year as a Masters student, I was completing the thesis proposal to my research, and I was confident that once I was able to meet and recruit participants, everything would fall into place. Early in that term, I had come across some people introduced to me by my supervisor who could possibly aid me with recruitment. Although I did not hear back from some, likely due to busy work schedules, I was not yet discouraged. In March of 2014, I had already made a breakthrough by speaking to a contact, a woman who worked at a community health centre. This was great for me for two reasons. First, even if I were not able to recruit strictly South Asian respondents, there was the possibly recruit people from other parts of the world, since that community health centre serves many different ethnic, cultural, religious and racial groups. Second, this community health centre is located not too far 
from my home, so the travel time would be much shorter, and meeting up with possible informants would be easier. At this point, I was also working on an application to the Ethics Review Board in Carleton, as well as working on my literature review, so I felt as though I was making great use of my time.

After speaking to the coordinator, she suggested I write her about a paragraph about my research in plain terms so she could send it out in her networks via email. She had a particular contact in mind who had access to the South Asian community in Ottawa. Unfortunately, after a few unsuccessful attempts, we were unable to reach this gatekeeper. The coordinator then emailed another one of her colleagues who also had access to this community, and thankfully, she was very keen on helping. Despite this, she was not able to provide as much help as she thought because she was very preoccupied during the late spring and summer months with other tasks. At this time, things began to unravel and a long waiting period began.

\section{ii. Rejection: bouts of disappointment}

[ri-jek-shuh $n$ ]: to refuse to have, take, recognize; to refuse to grant

By early June 2014, the research looked to be stagnant. I had tried to reach earlier contacts to see if they were available, to no avail. I had some success after meeting a former professor interested in helping with recruitment. This fell through, as he had sudden commitments to take care of. At this point, I began to wonder if there was something wrong with either myself or the project. It also did not help that many of my colleagues were making progress, or were in the field. After mass emails sent to several community health centres in the city, I had a breakthrough with a coordinator at the Ottawa Newcomer Clinic, which is affiliated with the Somerset West Community Health 
Centre (SWCHC). The Newcomer Clinic, she explained, works with government-assisted refugees, many from South and Southeast Asia, and helps them get access to health care. Because I was interested in working with South Asians, she felt that we start with the Bhutanese, Afghan and likely Tibetan clients. She also highlighted that the education levels were different for each group and to consider this when carrying out the research. She sent a copy of my Letter of Information, Consent Form, filled out forms from the Clinic and proof of Ethics Application completion to the SWCHC's Human Resources Manager. This process ended up taking nearly two months, until the end of August 2014. To me, progress was still progress.

Despite this foreshadowing of some headway, I want to highlight in this section that research is far from streamlined, and that disappointment and discouragement can lurk around any corner. In my case, feelings of disappointment and rejection appeared several times, even in the face of this new development. While I was waiting to hear from the Newcomer Clinic, I tried to make others contacts. For example, I made recruitment posters, and posted them at two South Asian convenience stores, and got in touch with an old professor from my fourth year, as mentioned. Although he was not able to help, he suggested I speak to another professor with links to the South Asian community. We met but were not able to find any respondents. At another time in October of 2014, I sent an email to an immigrant women's group in the city. They were not able to provide any help but told me to keep trying other immigrant-serving centres in the city. In November 2014, I was told to contact a prominent member in the South Asian community in the city who was eager to participate. Unfortunately, she did not contact me further. These events contributed to a question of my own research skills. 
Moreover, in attempts to 'save' the research, my supervisors gave me a few directions the research could possibly go in. One suggestion I was given was to do a series of interviews with owners of ethnic restaurants and ask questions regarding their food choices and ingredients in their meals, their possible health benefits, as well as cultural significance. This possible route was interesting because it could better link literature on food studies with human and health geographies more closely, to show how health can be understood through food, and as foodways change in the diaspora, then conceptions of health may change with it. Another suggestion was to do a review on reports and policy regarding health and migrants, as a way to address any gaps and to pose some suggestions on where health geography, postcolonial theory, and qualitative research may be contributable. This was useful in providing me with some of the context in understanding the situation of migrant, especially refugee health in Canada. One of the most important concepts drawn from this review was health literacy, mentioned in Chapter 1 . These suggestions were all great directions to take the research in, even though the project eventually picked up.

\section{iii. Redemption: resurrection of research [ri-demp-shuh $n$ ] - deliverance; rescue.}

As mentioned in the last section, I heard back from Siffan at the Newcomer Clinic, who was interested in the project but needed approval from SWCHC. After a long waiting period, Siffan suggested I work with the Clinic's health navigator, Abha. Abha, a Nepali woman, worked closely with the Bhutanese clients of the Clinic. This was beneficial for me because of the close relationship with the clients and her offer to serve as an interpreter during interviews. Abha was also very integral to moving this project 
forward, working hard to recruit from her networks. She was able to provide me contact with two other Nepalese women to interview, a young Bhutanese woman, and several elderly Bhutanese participants. Through Abha, I was able to conduct the interviews from the end of October to early November 2014.

\section{Emotions and reflexivity}

As mentioned previously, the discussion of emotions has not been fully unpacked in research analysis. One of the few pieces that discusses emotions in different stages of research is Holland (2007), who feels it is important for researchers to discuss emotional experiences, as emotions are tied with one's positionality, and "the researcher takes assumptions and emotions into and generates emotions in the field about the research" (p. 204). With referring to several of my field note entries, I want to demonstrate how these emotions have affected both my perceptions of research and my identification with it. Through these entries, I would like to illustrate the emotional work of qualitative research (see Woodby, et al., 2011). With this, I also want to refer to Noxolo's (2009) piece on the embodied production of postcolonial responsibility. To Noxolo, postcolonial geographic writing should take into account "the conditions of the pieces' production, in terms of the content of particular extracts, and in terms of the form in which they are written" (p. 57). Through this section, I want to go deeper into the phases of research I noted in the last section by presenting some extracts of notes I wrote during the duration of my fieldwork and speak on the embodied nature of the writing as well as the context. These extracts will help give a better picture of my research experiences and glimpses of some of range of emotional terrain covered. I use a different type of font (Lucinda Handwriting), closest to my handwriting, as a way to distinguish from the rest of my text. The first was a 
note written during the summer, just after I had heard back from a coordinator at the Newcomer Clinic. A short piece, it is a mix of anxiousness and excitement:

June 19/14

I just had my meeting with a coordinator from SWCHC. The Newcomer Clinic, affitiated with the Centre, is an interesting place. The best way I can describe it is that it smells like 'home. It reminds me of the smell of cumin, my favourite spice. I'm anxious to get started.

This first entry came from an interesting place, just after coming home from that first meeting. When looking back, my excitement to finally begin research may have had me try to unconsciously find links to the Clinic, almost as an act of emplacement. By noticing the faint smell of spice, I immediately connected this to the use of cumin in my own cooking. This initial excitement soon wore off, as approval took quite a long time. Siffan gave her support and encouragement during this delay.

July $27 / 14$

Tired of waiting.... anxious to get started!!!

The third major phase in the research was when things began to pick up, with two interviews in one day. I interviewed two participants, an elderly Bhutanese woman and her adult daughter in their home.

October $30 / 14$

After a late start to the day, I went to find disposable cameras but didn't think it would be too difficult. It's a dying art, that's sad [...] The house smells like my house, like food. Everyone stares, I'm a spectacle but I try not to mind the attention. Constantly family members are walking in, listening in, jumping with answers; the micro-geographies are loud and lively [...] On my way back, I noticed I smelled a bit like their home but it didn't bother me. In my 
own home, I'd be quick to run out but I think here it's proof I was there, a layer of skin when researching.

Re-reading this entry now, I notice how quickly I tried to identify myself with my participants and their home. Again, this excitement made me quickly notice the similarities in smell our homes had. The smell of their home which lingered on my clothes I was quick to say was evidence of my presence there, and I take a layer of this with me. Interestingly Noxolo (2009) encountered a similar problem with her experience working in Ghana, and assuming an unannounced kinship with Ghanaian locals, due to a larger sense of a Black identity. Noxolo's experience, though, went sour: "Ghanaians make it very clear, though not in an unfriendly way, that this is not your home, not permanently anyway...I have always taken the unity of Black people as self-evident but I have found it disturbing sometimes that Ghanaians do not. Sometimes this has lead me to hurt that people don't accept me as Black like them but rather white in a black skin" (p. 58). One of the points I can identify with Noxolo here is the assumption of a shared identity not only through smell but as I mentioned in my section on positionality, through a sense of immigrant/refugee status from my parents. I feel this highlights how the researcher should maintain a sense of distance from their research. A situation like this could create an issue of being unable to dissociate oneself from their research, and only finding data that favours assumed similarities. Outside of this excitement was my disappointment in struggling to find disposable cameras in my research. It was suggested by a community health worker that PhotoVoice might be interesting way to see where health takes place for participants, and Abha agreed it would be a good tool to use. However, just before the interview, other possible participants did not show up so 
PhotoVoice was later discarded.

The next entry was written in mid-November 2014, just after completing my last interview:

November $12 / 14$

Today was a double header. I had my interview with Leeza today, that went very well. She suggested someone I should speak to who would be helpful. I've been feeling disillusioned the past week or two. I haven't been really able to sit down, focus and write, even with interview data awaiting transcription. I don't know what the problem is exactly [...] Now that I have data, it's as though my motivation has dried. I don't look forward to doing interviews, which is usually my favourite part of research. Writing is becoming exhausting; I'm resisting the urge to bury myself in books to read. My relationship with my MA Thesis is almost evasive. I think the reality of how emotionally challenging grad school is getting to me.

This entry goes into more detail about the distance oneself should have with their research. Richards (1998) stresses that qualitative research needs to better explain what it means to be 'close' or 'distant' from research and data to ensure that they are being ethical and reflexive researchers. Richards proposes that "qualitative research needs a particular sort of distance because the researcher's relationship to the data is always changing and that relationship is itself data" (p. 320). Closeness to Richards, means having a combination of full knowledge of and access to one's data (p. 321). In my case, this could have likely been the fact that I had not transcribed my data, which lead me to want to take time away from the research. However, taking a break proved to be fruitful, as I was able to return to the data with a clear head. 


\section{Research methods}

With feminist methodologies highlighted earlier in this chapter, this section will discuss my decision to use semi-structured interviews as my primary method in data collection. However, before turning to this, it is worth noting that I had originally hoped to use three other qualitative methods: Body mapping, PhotoVoice, and walking interviews. My interest in Body Mapping (see Gastaldo et al., 2012) came as a way to visually represent the lived experiences of health and wellbeing of participants. I was also intrigued with body mapping, as it had not been used in geography, and I felt it to be an interesting visual method that the sub-discipline could adopt. However, due to time constraints, this method was let go. PhotoVoice (see Wang, 1999) was also considered, as a means to capture everyday health practices visually but some participants did not feel comfortable using cameras, and disposable cameras were difficult to obtain in large quantities. Another method I hoped to use was walking interviews (see Anderson, 2004; Jones et al., 2008; Carpiano, 2009; Evans and Jones, 2011; Bergeron, 2014). This was suggested by Abha, since many of the Bhutanese people enjoyed walking, it would be a way to conduct an interview. However, this fell through, as it was difficult to recruit many participants, and the Bhutanese participants were all interviewed in their homes. Because of these roadblocks, I ultimately used semi-structured interviews as my sole method of data collection.

\section{Semi-structured interviews}

In health geography and more broadly, health research in the social sciences, the movement towards using qualitative methods is still fairly new. One major point to keep in mind is the use of qualitative methods in health geography, as it is through qualitative 
methods, "health geography has introduced allied constructs of wellness, identity and place experience" (Kearns and Collins, 2010, p. 15), constructs which require an investigation of how these are experienced and expressed by informants. Most importantly, it was these shifts towards applying qualitative methods in this sub-field that were "instrumental in recasting the subjects of research as persons rather than patients" (Wilton, 1999, p. 256, my emphasis), emphasizing the role of participants as agents to their heath. Because the topic of health can be very sensitive, letting participants take questions in their own direction can provide some sense of control. For health geographers in particular, interviews can explore both the voice of an informant, as well as the spatial context. According to Dyck (1999), "the shift towards the subject's narrative and a concern with how behaviour and ideas are deeply embedded in place signal an engagement with the notion of recursive constitution of place and people, rather than conceptualizing places and spaces as unchanging 'backdrops' against which agency is performed" (p. 247). In other words, using a qualitative method, like interviewing, allowed me to see informants' narratives as place-based, having a particular geography. In this research, these place-based narratives refer to those particularly embedded within everyday life. Dyck also goes on to add that interviewing as a main research method for health geographers can help better understand migrant health, which "tends to adopt a culture-blaming perspective, instead of locating health experiences wSithin migration experiences" (p. 249). Attention to place through interviews is a major step taken by health geographers, Dyck argues, because of an ingrained interest in policy as well as health education and promotion (p. 247). Attention to narratives and lived experiences and geographies, then, can play a major role in how policy and promotion is formulated if 
given adequate attention by researchers.

Moreover, my decision to use semi-structured interviews was also a means "to collect a diversity of meaning, opinion, and experiences, [as] interviews provide insights into differing opinions or debates within a group but can also reveal a consensus on some issues" (Dunn, 2010, p. 302). Rather than coming into the interviews assuming responses would be similar based on a shared cultural background, I reminded myself that this would be seen as essentializing participants. For this research, I conducted four full interviews out of the eight participants. After some delays, I conducted two interviews at the end of October 2014, with a Bhutanese older woman and her daughter in their home. The first interview lasted over thirty minutes, and the second just under that, with about ten to fifteen minutes of introductions, initial questions of curiosity and exits. Through Abha, who served as interpreter, the aims of the research were explained to the participants. Some participants were curious as to what I was planning to do with the interviews, and Abha explained that it was going to be published in my thesis, which they were fine with. To protect identities, I used pseudonyms in the transcript.

My third interview was with a middle-aged Nepalese immigrant woman introduced to me through Abha. Much like with the first sets of interviews, time was dedicated in the beginning to introductions. This woman was open to noting her experience not so much in migrating from Nepal but with her work in the country with the rural population. The longest interview, it lasted just over an hour. My last full interview was with a well-known woman of Nepalese descent who has worked in health promotion among newly migrated groups in the city. Her interview lasted about forty minutes, and also suggested a woman from the Punjabi community worth contacting. 
In these last two interviews, power relations are important to consider. Because these two women held relatively high-paying jobs, it could be argued that these were elite interviews. Smith (2006) has noticed that when human geographers have noted the power dynamics in interviews, there has been little effort to discuss how 'power' is defined in these instances to show when a researcher is interviewing 'up', writing that "there is a need to overcome the current schism between conceptions of power within some of geography's methodological literature and the more complex ideas discussed in theoretical texts" (p. 644). Smith's solution is to use Foucauldian ideas of power, which conceptualize power as diverse and diffuse, coming from multiple sources, and constantly under negotiation. In my experience interviewing these two women, I did not feel threatened but took in what knowledge they were willing to give me about health in their community, and those in which they have worked with. Because I am also a younger researcher, I asked questions about their work, which seemed to have pleased them, and both were supportive in aiding in my work as well. This experience compliments one of Smith's suggestions: to find common ground with elite informants. Smith's advice for researchers is to consider their sense of positionality to better understand the nature of power in the space of an interview verses societal power structures outside of this space (2006, p. 652).

\section{Speaking in silences}

There are important points to consider when discussing these interviews. For one, I noticed a silence when interviewing the first participant, which made me feel a bit uneasy, as I was unsure if I asked an inappropriate question. Poland and Pederson (1998) feel that silence in interviews should not be seen as evidence of research failure but an 
opportunity "to begin to appreciate the multiple meanings silence may have" (p. 294).

Frankly, I feel that their idea of silence is problematic because it can lead to assumptions made by the researcher. For example, in my first interview, I noticed a silence when discussing religion, as this participant did not want to go into detail about her decision to convert from Hinduism to Christianity. In this case, I assumed this participant did not want me to assume her conversion was because of dissatisfaction with her former religion, or because the conversion was an emotional experience too difficult to share. Researchers in refugee studies, like Ghorashi (2008), feel that we should give silences a chance. Unlike the view that a silence in an interview is evidence of failure, Ghorashi believes that silences are evidence of a deeper layer of narrative and can actually help the researcher in learning more about a participant and their story. In my own interviews, paying close attention to this and subsequent silences made a difference in the themes that were pulled out from the interview transcripts. I found that the silence about religion to one participant was the silence and the frustration about work and employment to another other, both very prominent themes I did not anticipate surfaced in the interviews.

\section{Interpreters in cross-cultural interviews: power relations and ethics}

An important dimension of cross-cultural and cross-language research is the use of interpreters ${ }^{10}$. Because interviews were completed in a few weeks, learning to speak Nepalese was not feasible. However, as mentioned, Abha, the health navigator who works closely with the Bhutanese community, offered to handle translation during the interviews. Here, a factor to consider is the role of power in interviews involving interpreters. Shklarov (2007) notes that many qualitative researchers do not fully consider

${ }^{10}$ Translation refers written language and interpretation refers to oral communication (see Shklarov, 2007). 
the role of interpreters, mentioning that "the function of language translation or interpretation, in the view of many researchers remains the task of a 'fly on the wall'" (p. 530). Edwards (1998) notes something similar, and attributes this view to earlier works that "adopt a traditional 'value-free' and unreflexive stance towards the role of the interpreter" (p. 197). In other words, the consideration of power and power relations are absent from these renderings. Edwards (2013) links this stance to the fear of the academic researcher losing power in the face of the interpreter, since "academic researchers are not used to this dependency" (p. 507). In other words, there are some researchers who feel that interpreters may have a hidden agenda and are wary of their role in the research project. Shklarov writes that "the alienation of the translation function from the core of the research process can allegedly be aimed at both protecting participants from possible unethical translation and maintaining research integrity in the situations of perceived danger" (p. 530). In situations such as these, it is on the researcher to find an interpreter who is both aware of research ethics, as well as the agenda of the particular research project. I was not concerned a situation like this would occur, because Abha already worked with many of the participants to whom I spoke, so a rapport was already established. Another dimension to consider is the dual role of the interpreter, as someone who is a familiar face in the community, as well as someone is removed from it. Specifically, researchers need to consider the position of the interpreter in the community they are interviewing. For the most part,

people who routinely perform translations are most often, long-time immigrants and have been educated in the Western tradition. This makes them to some extent culturally distant from their non-English-speaking compatriots. Such situations can be further complicated by the fact that they may not share the same beliefs and values of native English speakers. (Shklarov, 2007, p. 531) 
As mentioned, with a close working relationship with many of the Bhutanese participants, I assumed Abha was already a noticeable and welcome figure. She was familiar in their homes and had stopped by for a meal, tea, or for an occasional walk. In this case, her being Nepali and well-educated did not appear to play a negative role in these interactions. Shklarov also makes the argument that interpreters, like Abha, may not consider the changes that language has undergone since leaving their home country: "they may not be aware of new words or constructs that have appeared in their mother tongue, or new meanings of old words or expressions" (p. 531). Nepali immigrants, like Abha, and Bhutanese refugees are unique in this situation, as both groups migrated to Canada at roughly the same period, and there were likely some interactions between Nepalese and Bhutanese people prior to migrating to Canada

Shklarov's (2007) main argument in the article is that the interpreter has 'double vision' when interpreting interviews: to convey both the academic's and the interviewees' constructions of knowledge. The author refers to the linguistic and anthropological concepts of emic and etic knowledges to make this distinction, with emic "pertaining to culturally-specific issues" (p. 532) or knowledges, and etic as "broader universal terms" (p. 532). Viewing this interpretation as not so much a process where some knowledges are inevitably lost but as a distinction between emic and etic can be a way to reassure and comfort concerned researchers. I found this distinction apparent in Abha's style of interpreting: I would read aloud the interview question that was then translated into Nepali for the interviewee. However, Abha would then explain to me exactly what was said, if words in the question were changed in order to better translate them. Abha would then translate to me in English the interviewee's narrative. At times, interviewees would 
even correct her if words did not translate properly. I appreciated her honesty in the interpretation process because it allowed questions to be better understood and provide data that I am certain would not have come up had interview questions been transliterated. The corrections made by participants were also interesting to me because they signalled a participant's strong effort to have their views and stories expressed in their own terms. In these instances, one could argue that participants resisted being spoken for and othered in academic research. More discussion in the literature on this situation could greatly benefit works on interpreters in qualitative research, especially with more and more geographers working globally, and with people of various diasporas in the West.

\section{Micro-geographies: the role of place in semi-structured interviews}

Before interviewing, I wanted to take into account the role of place. I read articles by Elwood and Martin (2000) and Sin (2003), which discussed the importance of 'placing' interviews. Elwood and Martin see interview sites as micro-geographies, which "reflect the relationships of the researcher with the interview participants, the participants with the site, and the site with the broader sociocultural context that affects both the researcher and participant" (p. 650). The micro-geographies of interviews do not only highlight the importance of location but also sense of place, people's reactions and attachments to particular sites. From the interviews, I noticed with the two Bhutanese

women I spoke to, the interviews taking place in their home was significant in terms of the narratives they shared. The first informant discussed some of her memories from living in refugees camps in Nepal, giving rich detail of day-to-day life there, and comparing this to her life on a farm in Bhutan. This is a narrative to which I felt was 
connected to and brought up vis-à-vis the interview site.

In addition, Elmwood and Martin point to the relationship between identity and place as also important to interview sites. These micro-geographies highlight "spatial relations and meanings, where multiple scales of social relations intersect in the research interview" (2000, p. 649). These spatial relations can refer to different and multiple identities and how these may shift in spaces, especially in the presence of others. With the first participant, conducting interviews in her living room, with family members present, seemed to help her recall some of her pre-migration memories. At one point, she and another family member went back and forth between their memories, talking about the use of kerosene for fuel and their access to electricity. A conversation like this may not have occurred had the interview been conducted elsewhere. My third interview was quite a different location, which I feel made the interaction not as free as with the first two women. In a room in the Newcomer Clinic, this participant was able to share some of her work with rural Nepalese women but likely because of the location, the interview felt much more formal. My last interview took place in a coffee shop in downtown Ottawa, around noon. I felt in this instance, the location to be a good choice for the informant and myself as the traffic of people distracted them from the conversation we were having, and it took her away from her office. Perhaps in these two interviews, choosing a location that was not linked to these women's identities eased the interview situation for the both of them, in places removed from their daily lives.

In all, Elmwood and Martin stress that the "reflection on the micro-geographies of interviews is a process that starts before the actual interviewing begins, and continues the research and analysis" (2000, p. 656). Before beginning interviewing, I had already 
assumed that participants would choose the sites in themselves to meet to ease the situation but what I also learned was that when analyzing the data, the role these places would play. For example, after my first two interviews, I had asked the women if I was able to take a photo of their living to use in my research, to which they agreed, and even offered to pose. Looking back at this photo helps me to recall what I was feeling during these interviews in the writing portion of research. The photo still serves as a piece of interview memorabilia.

\section{Ethical Issues in the Research}

Qualitative research can be a very informative but risky affair, and through the involvement of human participants, many ethical issues need to be discussed to keep participants safe. Guillemin and Gillam (2004) understand ethics to be composed of two parts: "(a) 'procedural ethics', which usually involves seeking approval from a relevant ethics committee to undertake research involving humans; and (b) 'ethics in practice' or the everyday ethical issues that arise in the doing of research" (p. 263). As procedure, I completed an ethics application through Carleton University's Ethics Board, and interviews took place well after clearance was received. Due to several shifts in methods and target groups, further changes to protocol and revisions were made in a timely manner. The ethics application given to would-be informants contained a Letter of Information and a Consent Form to read to better understand the research. Abha, who served as interpreter for Bhutanese participants explained the project, as well as the rights of participants if agreeing to contribute. In these forms, I explained that participants had the right to end or withdraw their participation within an agreeable date, and that I would 
guarantee to destroy any data attached to them. I also explained that with their consent, interviews would be audio recorded. All but one participant was given a pseudonym to protect their identity in this thesis or any future publication. Although some were interviewed in the presence of others, like friends or family members, none voiced any concern with this.

The second component of ethics, as mentioned, is to consider issues of ethical concern that may arise in research practice. For participants, especially Bhutanese participants, the psychological risks were very present. For one, sharing memories and stories about their lives both migrating from Bhutan and living in refugee camps in Nepal for over a decade were not easy for all. I did feel slight guilt at some points during some of the interviews when these were shared, wondering if participants felt compelled to share these with me. Other psychological risks were related to participants who discussed their experiences in losing employment after settling in Canada, being unable to run a shop, or work on a farm, as they had previously.

Some of the social risks for participants had to do with disclosure of their identity. Most of the participants' names were changed ${ }^{11}$. However, with the Bhutanese participants I spoke to, they were interviewed in the presence of either other participants or family members, and these situations greatly comprised the confidentiality promised in the Consent Form. When conducting research in small communities, Damianakis and Woodford (2012) advise researchers to consider the nature of particular small communities at every step of the research process. Damianakis and Woodford also refer to the work of Ellis (2007), who stressed the importance of considering the "ethics of what to tell". In my research, information such as addresses or neighbourhoods in which

${ }^{11}$ Actual names of gatekeepers were kept 
participants lived were left out of the context as a way to better conceal their identities. The inclusion of pseudonyms, ethnic backgrounds, nationality, and age range I felt to be the best way to both protect participants, as well as distinguish them from each other. Through all of these measures, I felt I did my best in considering the ethics in my research.

\section{Analysis of Data}

The main goal in this research was to examine how the concepts of health and wellbeing could be understood through the everyday routines of Nepalese and Bhutanese migrants. Through data collection in the form of semi-structured interviews, the results were split into five sections. The first discussed how health and wellbeing were defined by participants, their similarities and differences. The second spoke to noting specific practices associated with health. In this section, conceptions of the health benefits of walking are explored. In the third section, much like the first, narratives on the benefits of cooking, and decisions to use particular food items were unpacked. The fourth section examined links between work, wellbeing and one's sense of worth or self-esteem. This section is particularly striking to me because it was not an area of everyday I had considered before interviewing. Lastly, through therapeutic networks, discussing the role(s) of the neighbouring communities is highlighted. This section is also the space to discuss the limited attention negative therapeutic experiences have received in the literature. All of this will be made clearer in the next chapter discussing research results. 


\section{Conclusions}

This chapter discussed the methodology and methods that were employed in this research. The research was structured through the application of feminist methodologies, which are concerned with addressing not only everyday life but situations related to power issues, especially considering the opinions and assumptions the research may carry to the field. These issues, in the feminist perspective, have a role in how knowledge is viewed, validated, produced, and applied in research. The goal, then, is to make these processes visible and for the researcher to critique their own role and motivations for research. I have discussed these concerns by highlighting four components of feminist methodologies, namely, positionality, power, subjectivity, debates concerning emotions and reflexivity in the research.

I then turned my attention to debates surrounding reflexivity. My aim in this section was to turn my attention to the many roadblocks encountered in my recruitment phase, shown through written entries in a field notebook, as a means to better understand reflexivity, a concept given many definitions. To do so, I borrowed from the work of Pillow (2003) and structured my personal palimpsest of reflexivity, composed of rejoice, rejection, and redemption. An important point here is that reflexivity has many layers, and is unique to each researcher. Another is that research 'failure' and success happen in many different stages, so many of the layers of my palimpsest fold onto each other at times. In other words, research can be a messy affair, and a detailed chart of these phases was put into a table. From reflexivity, the research methods were discussed. After switching between Body Mapping, PhotoVoice, and walking interviews as secondary methods, I ultimately used semi-structured interviews as my sole method for data 
collection. Four one on one interviews and one group interview were conducted through the assistance of an interpreter, as a means to better understand perceptions of health and wellbeing, and view these narratives as health knowledges and lived experiences. In these interviews, I had also obtained participants' consent, emphasizing their confidentiality, listened closely to their responses, and mentioned their ability to withdraw from the research. Once all interviews were completed, I analyzed the data and organized them into five themes, using direct quotes from the interviews to keep participants' voices and opinions, as well as reflecting on my own interpretations of these findings. These findings, in detail, will be illustrated in the next chapter. 


\section{Table 1: A timeline of research failures and successes}

\begin{tabular}{|c|c|c|}
\hline Month/Year & Recruitment Method & Success/Fail \\
\hline January 2014 & $\begin{array}{l}\text { Email to a health promoter at a community } \\
\text { health centre }\end{array}$ & $\begin{array}{l}\text { Fail: she did not reply } \\
\text { to the email }\end{array}$ \\
\hline February to March 2014 & $\begin{array}{l}\text { Email and meetings with coordinator at another } \\
\text { community health centre }\end{array}$ & $\begin{array}{l}\text { Mixed: expressed } \\
\text { interest and sent } \\
\text { emails along her } \\
\text { network but there was } \\
\text { no feedback }\end{array}$ \\
\hline May 2014 & Email to a contact from the contact above & $\begin{array}{l}\text { Fail: did not reply to } \\
\text { my message or that } \\
\text { from the coordinator }\end{array}$ \\
\hline May 2014 & $\begin{array}{l}\text { Received email from a contact linked to second } \\
\text { contact noted }\end{array}$ & $\begin{array}{l}\text { Mixed: expressed } \\
\text { great interest, } \\
\text { especially with her } \\
\text { links to the South } \\
\text { Asian community } \\
\text { Had time constraints }\end{array}$ \\
\hline May 2014 & $\begin{array}{l}\text { Sent email to South Asian women's group in } \\
\text { Toronto to ask for any Ottawa contacts }\end{array}$ & $\begin{array}{l}\text { Fail: suggested } \\
\text { contacts in Montreal }\end{array}$ \\
\hline May 2014 & Contact from a University of Ottawa professor & $\begin{array}{l}\text { Successful: suggested } \\
\text { possible avenues }\end{array}$ \\
\hline June 2014 & $\begin{array}{l}\text { Sent emails to all of the city's Community } \\
\text { Health Centres }\end{array}$ & $\begin{array}{l}\text { Successful: heard } \\
\text { back from Somerset- } \\
\text { West Community } \\
\text { Health Centre } \\
\text { (SWCHC) and the } \\
\text { Newcomer Clinic }\end{array}$ \\
\hline July 2014 & $\begin{array}{l}\text { Met with a former professor of South Asian } \\
\text { decent to suggest contacts }\end{array}$ & $\begin{array}{l}\text { Mixed: due to time } \\
\text { constraints on his end, } \\
\text { I was not able to } \\
\text { interview any women } \\
\text { he suggested }\end{array}$ \\
\hline July 2014 & $\begin{array}{l}\text { Met with coordinator from SWCHC and } \\
\text { Newcomer Clinic to discuss project; names a } \\
\text { fellow worker to work alongside with } \\
\text { Target group: Bhutanese refugees from Nepal }\end{array}$ & $\begin{array}{l}\text { Success: project was } \\
\text { approved but awaiting } \\
\text { final say from the HR } \\
\text { of the Centre }\end{array}$ \\
\hline July 2014 & $\begin{array}{l}\text { Posted recruitment poster at a South Asian } \\
\text { convenience store }\end{array}$ & $\begin{array}{l}\text { Fail: did not hear back } \\
\text { from any interested } \\
\text { participants }\end{array}$ \\
\hline July-August 2014 & $\begin{array}{l}\text { Met with a professor in Carleton for possible } \\
\text { contacts }\end{array}$ & $\begin{array}{l}\text { Mixed: time } \\
\text { constraints; away for a } \\
\text { trip }\end{array}$ \\
\hline
\end{tabular}




\begin{tabular}{|c|c|c|}
\hline August 2014 & $\begin{array}{l}\text { Met with coordinator from the Newcomer } \\
\text { Clinic to sign forms }\end{array}$ & $\begin{array}{l}\text { Success: project fully } \\
\text { approved }\end{array}$ \\
\hline September 2014 & $\begin{array}{l}\text { Asked an old South Asian classmate for } \\
\text { possible participants }\end{array}$ & $\begin{array}{l}\text { Fail: she could not get } \\
\text { women to participate } \\
\text { in the project. She also } \\
\text { noted language } \\
\text { barriers as a problem }\end{array}$ \\
\hline October 2014 & $\begin{array}{l}\text { Sent email to Immigrant Women's Centre in } \\
\text { Ottawa for possible contacts }\end{array}$ & $\begin{array}{l}\text { Mixed: suggested I } \\
\text { speak to immigrant } \\
\text { groups in the City }\end{array}$ \\
\hline October 2014 & $\begin{array}{l}\text { Suggested by coordinator to email a contact } \\
\text { who has links in the South Asian community }\end{array}$ & $\begin{array}{l}\text { Mixed: expressed } \\
\text { interest but has yet to } \\
\text { hear from her }\end{array}$ \\
\hline October 2014 & $\begin{array}{l}\text { Conducted } 2 \text { interviews with Bhutanese } \\
\text { women }\end{array}$ & Success \\
\hline November 2014 & $\begin{array}{l}\text { Conducted } 2 \text { interviews with Nepalese women } \\
\text { Interviewed a mixed group (approximately 10) } \\
\text { of Bhutanese men and women ( } 5 \text { people spoke) }\end{array}$ & Success \\
\hline $\begin{array}{l}\text { November } 2014 \text { - } \\
\text { December } 2014\end{array}$ & $\begin{array}{l}\text { One participant suggested I contact a prominent } \\
\text { member of the Punjabi community in the city; } \\
\text { provided email }\end{array}$ & $\begin{array}{l}\text { Mixed: contact } \\
\text { expressed great } \\
\text { interest and suggested } \\
\text { that she may be able } \\
\text { to bring other } \\
\text { interested women } \\
\text { Update: did not return } \\
\text { second email. }\end{array}$ \\
\hline
\end{tabular}

NOTE: This table is a summary and timeline of the various networks and strategies used to recruit participants for my thesis work. To create this chart, I wrote down important moths and dates with names attached to key gatekeepers, as well, I searched through my university email inbox for others contacts I made over the last year. Although this project was eventually picked up and interviews were done in October and November of 2014, I still would like to visually demonstrate the long process of participant recruitment. 


\section{Chapter 4: Research results}

\section{Introduction}

This chapter will discuss the findings from the semi-structured interviews I conducted with immigrants from Nepal and government-assisted refugees from Bhutan. In this chapter, I plan to answer two of my research questions: i) what practices or routines do individuals perform every day to maintain their health, and, ii) where are these health practices taking place? Through these research questions, I seek to demonstrate the extent to which 'health' is a part of the everyday life of the participants but also hope to draw connections between these everyday geographies of health and other spheres of life, such as work and employment. To organize these results, I have split the chapter into five sections, each corresponding to a major finding or theme. First, I describe the notions of 'health' and 'wellbeing', as seen through the experiences of participants themselves. Second, I look at the role of walking as a health practice, and not merely a mundane act of movement. Here, walking appears to be linked to a sense of independence. Third, I examine some of the notions of food as a cultural item, and note the food items which participants regularly consume as part of maintaining their health. Typically, many informants saw traditional, South Asian food ingredients and meals as being 'healthy', even if their nutritional value may be questionable. As well, I will touch upon the concept of cleanliness which was mentioned by a few participants, and its link to understandings of food and health. Fourth, I note the prevalence of work, income and employment as related to conceptions of health and wellbeing. What is intriguing about this finding is that several of the participants noted that the issue of work is not widely discussed in social circles in these communities, yet this subject matter was very present 
in the interviews. Lastly, I end with highlighting some of the perceived therapeutic and untherapeutic roles the neighbouring community plays in providing support and health advice, as both positive and negative. Overall, the aim of this chapter is to highlight how health and wellbeing impact different facets of life, and shed light on some of the cultural, social and economic barriers for recent migrants adjusting to life in Ottawa.

\section{Refugee or Immigrant: the contextual role of immigration status}

In Table 2 below, I have organized each speaking participant by pseudonym, age range, gender, and national and immigrant status for convenience.

Table 2: Participant table, organized by age, gender, nationality and immigrant status

\begin{tabular}{|c|c|c|c|c|}
\hline Participant & $\begin{array}{c}\text { Nepalese or } \\
\text { Bhutanese }\end{array}$ & $\begin{array}{c}\text { Immigrant or } \\
\text { Refugee }\end{array}$ & Age range & Gender \\
\hline Tsering & Bhutanese & Refugee & $(60+)$ & Female \\
\hline Norbu & Bhutanese & Refugee & $(20+)$ & Female \\
\hline Radha & Nepalese & Immigrant & $(35+)$ & Female \\
\hline Tenzin & Bhutanese & Refugee & $(60+)$ & Male \\
\hline Sangye & Bhutanese & Refugee & $(60+)$ & Female \\
\hline Yeshey & Bhutanese & Refugee & $(60+)$ & Female \\
\hline Leeza & Nepalese & Immigrant & $(25+)$ & Female \\
\hline
\end{tabular}

The most important point to take away from this table is that there is a difference between participants immigrating from Nepal, and participants who have come to Canada as refugees, originally from Bhutan. In addition, it is important to recognize that their 
varying migration routes and experiences do have a profound effect on their daily lives here in Canada. When considering the role of health in the everyday, we must remember that the experience of fleeing Bhutan and living in refugee camps in Nepal can both be traumatic emotional experiences. As well, it is important to consider the possible role of nostalgia in their narratives. With many issues in adjusting to life in Canada, like distance from other family members, and adapting to a new chaotic environment with a different social climate, a yearning for one's past of familiarity is expected. Discussions with most of the Bhutanese informants highlighted for the most part, positive memories of living in refugee camps in Nepal. In one particular interview, a participant shared memories of being able to walk around independently because of the area's familiarity, and I was told that the refugee camps had access to electricity. These examples demonstrate how "the past has the power to bond communities" (McDowell, 2009, p. 62). At the same time, this constant referral to the past to understand the present may also be read as using memory to "operate as a mode of resistance" (p. 62), to use these as coping devices. Attention to this nostalgia and memories from Nepal and Bhutan is important, as it helps us understand the context of their narratives.

Much like taking into account the migration history of the Bhutanese population in particular, as well as considering the role of nostalgia in narratives, it is also crucial to consider the socio-economic situation informants have once resettled in Canada. The Bhutanese are primarily supported through the Refugee and Resettlement Program. According to Citizenship and Immigration Canada, refugees are financially supported and provided assistance to find permanent housing, and "the amount of monthly income support provided to government assisted refugees is based on the current provincial social 
assistance rates. It provides the minimum required to cover only the most basic food and shelter needs until the refugees are able to support themselves" (CIC, 2015). However, finding long-term employment can be difficult for this population, especially among older Bhutanese people because of the lack of recognized education, as well as language barriers. Factors such as migration, nostalgia, education and language all play a part in shaping and providing insight into participants' narratives. The following discussions will highlight how these factors also impact how participants conceptualize both health and wellbeing in their everyday lives in Ottawa.

\section{Perceptions of health and wellbeing}

\section{Health}

To better understand the routines and practices that participants perform to maintain their health on a daily basis, it is important to first examine what they think 'health' means and encompasses. My interviews with Radha, Tsering and Leeza demonstrate that understandings of health can be varied. I begin with providing a transcript of my interview with Radha, a Nepalese immigrant woman of middle age. She felt that health was an individualized task, something that she maintained on her own. I saw this as possibly relating to her migration history, entering Canada from the United States, which had likely exposed her to this individualized lifestyle. She explained:

Asli: Do you think health is a part of our everyday lives?

Radha: Absolutely. Health comes first no matter what. If you are healthy, if you are wellbeing, you can focus on other things. So if you are not healthy, then your whole time is consumed, "Oh, I'm not healthy, I have to see a doctor..." You could imagine, right, how much time it takes for you to be that. So health is one of the 
main things we need to have so we need to focus on it, because if you are healthy, then you can be a wellbeing, you can contribute to other things.

Radha also mentions her perception of health in her community, the Nepali community:

Radha: [...] my community, we are not serious about our health. We would rather lie down in bed and get people's attention, saying "Oh, how are you feeling?" like that but we won't take some kind of medicine. And we need to. But for me, health is a priority.

Tsering, an older Bhutanese woman, has lived in Ottawa for over five years, saw health

differently. Her ideas of health seemed to link with her age:

Asli: Do you think health is a part of our everyday lives?

Tsering: I think about my body, and I'm getting old, so I have to think about health. I cannot do much. I'm worried about my health, and you're slowing down as you're getting older. Everybody is very independent around here so it's a little bit separating. This society is very individualistic. Children take care of themselves, so it's very separated. In our culture, the children look after the parents but it's not the same in this place [...] Sometimes when I look at myself in the mirror, I feel worried, you know. There's meat on my cheeks, coming down, not up as much that I feel like I'm weakening, I'm getting old.

However, another Nepalese immigrant woman mentioned nutrition and genes as part of her understanding of health. Leeza has been living in Canada for over five years, emigrating from Nepal. She came to the country as a skilled immigrant fluent in English. She has experience working with Bhutanese migrants as well as other recent migrant groups, such as Punjabis, Somalis and Latin Americans in numerous health promotion programs. Leeza has also led three community kitchen events and a diabetes risk assessment program with the Centretown Community Health Centre:

Asli: Do you think health is a part of our everyday lives?

Leeza: Definitely. I mean if you deny health as being part of your daily life then you face the consequences later in life and anytime, especially because of the genetic dispositions we have as South Asian, because of our sedentary lifestyle, of our diet and we are very prone to a high risk of diabetes so it's only a matter of 
time when it can decide to catch up with us if we don't pay attention to our health now. I definitely think it's a huge important part.

Although somewhat varied, the common thread between the narratives of Nepalese and Bhutanese migrants was the role of time in understanding health. As shown, health is an important part of our daily lives, the present. However, its real significance comes from its role in determining our future: our longevity, mobility, etc. Health, then, is not temporary but temporal, a lifelong task. For participants who are recent refugees, like Tsering and Norbu, perhaps maintaining their health can be seen as an arena for expression, a sense of agency, especially where other facets of their life, such as access to employment, are uncertain. For someone like Tsering, this sense of uncertainty can be even more stressful when dealing with aging, as her body is tiring, as Tsering continued to walk to keep her body in motion, despite this pain; this perseverance in the face of pain helps her move forward to the future. Tsering's narrative also demonstrated a fear of the state of her health in the future. She worried that Western society, and the emphasis of self-sufficiency and independence may influence her family, which may leave her all alone. In other words, acculturation in the future is on the mind of Tsering, which she believes will change the family dynamics. Radha's idea of health appears to be the most expansive, as she felt health should be given our full attention. Radha connected health to work, food practices as well as our own mental health. In contrast, Leeza as a health promotion worker saw health as internally temporal. In other words, she is more concerned with how our body's organs are going to be affected by the food and exercise (or lack thereof) in our lives. Leeza's mention of race is important here, as well. By looking at the types of foods eaten in South Asian meals, predominately the use of ghee (clarified butter) which appears to be a major contributor to diabetes in this population. 
Leeza seemed to suggest that South Asian people need to consider this to ensure a long healthy future. Unlike Tsering and Radha, Leeza did not mention how health can also be emotional, or connected to other facets of life, like employment. From Leeza's narrative, health appears to be something that comes externally from our food choices and exercise, which later affects us internally, through our organs. In summary, health becomes temporal when its maintenance is understood to be a task to ensure a brighter future, in the face of uncertain circumstances. Considering how health seemingly touches on other areas of one's life is important. In the section, I will be taking this same notion when considering wellbeing.

\section{Wellbeing}

As mentioned in the review of the literature, the literature on wellbeing consists of both works related to social and welfare geographies on housing as well as income security, and literature is linked to emotional geographies and therapeutic landscapes (Atkinson, et al., 2012, p. 23). However, it is important to keep in mind that asking questions about wellbeing may be difficult as it is sometimes seen as synonymous with health (see Healey-Ogden and Austin, 2011). I had initially assumed that most participants would primarily refer to the emotional component of wellbeing but noticed that both streams of wellbeing were expressed:

Asli: I forgot to ask this question earlier: health versus wellbeing?

Tsering:[...] I still feel healthy in terms of what my age is. But sometimes in my heart, there's things going on. I'm really worried about my immediate family. I find my health is good but in terms of my overall 'feeling well', I'm still stressed about the fact that I have to worry about myself, my husband, my older kids and my grandchild, because who's going to take care of them once all those who are smart move away from the house? [...] so what will happen to me once the younger kids are in high school and learning fast and once they get married and 
move away? What would happen to my older children who are having trouble with the language school? How are they going to progress?

Tsering discusses both emotional and economic notions of wellbeing. The double bind shown here is the benefits of learning English to help with job-seeking while at the same time, a mastery of English may also lead to social mobility, with family members taking off in different directions. Perhaps Tsering's sense of wellbeing would be broken with family members living separate lives.

Radha saw wellbeing in a similar, collective sense:

Asli: OK, so how would you define health and wellbeing, and are there similarities and differences between the two terms?

Radha: [...] wellbeing is a lot to do with emotions, feelings and it's a little different than when you say, 'I'm healthy'. That [wellbeing] has to do with more like the mental and emotions [...] You know when we say that you are healthy, you can make your family healthy and your group healthy [...] and very importantly, your personal health would have to be wellbeing to make your community. So if you are not, then how can you make others, right?

I noticed a stark difference in my interview with Leeza. She seems to see wellbeing as very distinct from health, an area for concern in health promotion. To her, health consequently overshadows attention to wellbeing:

Leeza: $[\ldots]$ wellbeing is basically how one leads their lifestyle, so that defines the wellbeing of an individual. And that's where I think most of the challenges lie, is because we often feel that wellbeing in Nepalese communities, people that belong to the rural community oftentimes, health issues are not 'health issues', especially health issues like diabetes and other illnesses that can be avoided, are not that existent because they walk a lot. They do physical activity, they do physical labour, like farming, walking a lot between the proximity of villages [...] In urban centres, like in the capital (Kathmandu) or in urban areas, people oftentimes they're closer, so their physical activity is limited by geography as well. Then they have a false sense of wellbeing thinking, 'Well, my parents never experienced it, so I'm never going to experience these diseases' [...] 
These responses overall portrayed wellbeing as something that is not innate but to be consciously achieved. For Tsering, wellbeing was an expansive sense of health, which included the good health and perceived happiness of all of her family members. This greater sense of wellbeing to her seems to be something that needs to be achieved.

Radha's definition of wellbeing was similar to Tsering's mention of the emotional wellbeing of herself and her family, as she, too, believed that wellbeing needs to be achieved. In her case, one needs to consciously work on themselves and find their niche through hobbies, like sewing. Once someone nurtures themselves emotionally, then they can move to helping others, expanding this positive sense of wellbeing. This seems to be the opposite of Tsering, who understood wellbeing as working from the outside, then in, first, within her family, then to herself. Here, a strong sense of self is needed to maintain not only oneself but also their family and broader newcomer community.

Lastly, Leeza's narrative mainly touched on the importance of attention to premigration routines and work habits. One major point to take away from this is that due to difficulties some Bhutanese people have in accessing work in Canada, as their experiences in working on farms, a major source of daily exercise, do not seem to be transferable. This economic view of wellbeing writes against Leeza's implying that wellbeing is totally ignored by Bhutanese migrants once arriving in Canada. Barriers to employment appear to have an effect on one achieving a desired sense of wellbeing.

\section{Steps to wellbeing: walking and mobile geographies of health}

From the interviews, I noticed a motif of the importance of daily walking, as both a form of exercise and as a way to stay connected to people. Attention to walking and 
everyday geographies should be noted by medical geographers because both the daily movements of individuals and the more enduring migration of people during the lifecourse are crucial in understanding the intersections between human health and spatial settings" (Gatrell, 2013, p. 98). However, attention should also be paid to how walking can be related to one's socio-economic status, since walking is a means of transportation for those who do not have access to cars. It is important to also consider that due to language and economic barriers public transit may also be difficult for new migrants to navigate.

For Tsering, walking served as a way to demonstrate her resilience to her aging body. In this sense, walking helped her cultivate a sense of both health and wellbeing:

Asli: What do you do every day to maintain your health?

Tsering: I walk around, even though my legs hurt, I try to walk around a little bit I try to go to different places. If people invite me over, I go to different people's houses and that's my walking.

She also mentioned later in her interview that walking is not a solitary task:

Tsering: Me and my husband would go for a walk sometimes and it's much better but if I'm left alone, then I might get sad.

Even with pain in her legs, Tsering was determined to stay mobile and remain connected with the Bhutanese community in her neighbourhood.

For Norbu, walking was not so much connected to keep close with the Bhutanese community as much as it was a mode of transportation to and from her language school. She also noted how the seasons affected her mobility, with large amounts of snow isolating her:

Norbu: Mine is school too. I walk over there and that's my exercise. And I do different things at school [...] In winter time, it's going to be hard. I cannot walk, 
do anything in the winter; I feel it isolates me. Whereas when it's nice, I can walk around, do different things. It's going to be hard to move around.

For Radha, walking had a more nuanced significance in her daily life:

Radha: I walk, I walk a lot [...] and when it's summer, I spend a lot of time in the park but mostly I'm outside. And I love nature, so maybe this is one of the things [...] The other thing is just to keep ourselves going and motivated just for the encouragement of us, we have to develop our own hobby, we have to find our needs. For me, I like to go out a lot, even just for a cup of coffee, I like to go out, I like to be with somebody and talk and interact, and when I come home, I'm so relaxed and fully charged. I do more than that. Even at work, I don't spend too much time, you know, I'm just walking around. And even taking the elevator, I like to take the stairs [...] So within that 7/8 hours in which I'm working, I have to find a way to keep myself fit and keep myself healthy [...] I see movies, I meet friends. Not always 'out-out'.

However, viewing walking as a form of health maintenance is not always so clear. To be able to freely walk, one needs to also have the language skills to travel around:

Asli: Question just on the particular places that you go to that you think are healthy? Places that you feel good?

Sangye: If I could go everywhere, I would feel healthy (laughs). If we could roam around somewhere, of course, we would feel healthy in the heart.

Abha to Asli: I just asked them why they were related: going out and education.

Sangye: I feel good wherever I go. I haven't been to many places in Ottawa.

Yeshey: Because she is not educated, she cannot go anywhere.

Sangye: If you were educated, you would go to different places and you would want to know what purpose is for going there, so you can enjoy. When you don't know where you're going, then what's the point of going there? I have been to parliament, I have been to different places to see them.

Abha to Asli: They've been to the Tulip Festival, that's what she's saying.

Overall, this section examined how health can be practiced in everyday routines, like walking, which forces researchers to not overlook these ordinary geographies in promoting feelings of health and wellbeing in everyday lives. For Tsering, walking is a 
mode of transportation and evidence of resilience, despite pain in her legs. I understood this activity as something that is able to illustrate that even within pain, a sense of relief and wellbeing can be found (Doran, 2014). Tsering also noted that for her, walking is not a solitary activity, as she prefers to walk alongside her husband. Norbu mentioned that when winter approaches, she can no longer walk to school, her main form of exercise; she did not mention if she would consider taking the bus instead to school. Tsering saw winter in a bleak light as well, stating: "Now there was a time when everything went colourful and now everything is gone".

For Radha, walking appeared to be used for exercise, as well as a way to maintain close relations with others, like her work colleagues, friends and nature. What should be kept in mind from these accounts is that the emotional as well as economic underpinnings of walking as an everyday health practice. Through Tsering's and Norbu's interviews, we can see how both women also rely on walking as a source of transportation, and this can be difficult to do during the winter months. This point needs to be taken into account when considering the role of walking as an everyday health practice, especially for recent refugees.

\section{Consuming health: the role of food, cooking and cleanliness}

Another prominent theme from the interviews was the importance of food in maintaining one's health. Much like the responses to walking, the significance of food was nuanced, related to ideas of health but also cultural identity and migration experience (Valliantos and Raine, 2008). As with walking, there needs to be a consideration of the socio-economic status of informants, and how this may influence food habits. The 
Bhutanese informants I spoke to were very keen on preparing traditional Bhutanese meals at all times, with little borrowing from Canadian foodways, which is also evident in the literature (Kiptenness and Dharod, 2011). The main food items were the same, with rice, daal $^{12}$, saag $^{13}$, and achar $^{14}$ noted as staples in the Nepalese and Bhutanese diet. What is also interesting is that not all foods they mention as being 'healthy' were necessarily healthy in the nutritional sense but important as a cultural staple. For instance, many participants mentioned their consumption of $g h e e^{15}$, which is known for its rich taste and high fat content. One participant, Sangye, joked about drinking alcohol back in Bhutan and it being good for her health. In this instance, health seemed to blend into wellbeing, an emotional satisfaction of eating food of familiarity. Below, I show other examples of the importance of food and cooking to conceptions of health and wellbeing.

For Tsering, food appeared to be symbolic, related to maintaining a Bhutanese identity, as she has not adjusted to Canadian food:

Tsering: I eat rice, cucumbers, makes vegetables. I try to eat on time. I don't really like Canadian food, I'm not Canadian yet. Nepali people eat rice, daal, vegetables, and that is what I like to have.

She also discussed her memories of food back in Bhutan:

Tsering: I remember my farm and the growth of different vegetables that we used to have - wheat, barley. We never had to buy anything in the stores in Bhutan. So all different kinds of vegetables: potatoes, chillies, eggplants, soybeans. We never had to buy anything, we had our own farmland. There used to be rice patties big and thick, it was beautiful. The certain kind of rice used to look different in colour, bluish kind of colour. and it was really expensive and really tasty [...] If you cook that rice here, it would smell to the next building, it was that much of a smell. It's hard to find that kind of rice here [...]

12 lentil soup

13 leafy greens

14 pickles

15 clarified butter 
Tsering went into detail of her memory of Bhutan and her family's farmland through mentioning of food, which can show the importance of food to Bhutanese identity, especially while in the diaspora. I also saw her family farm as a major place associated with her health. In contrast, this memory also had an economic component related to food access. Once moving from the farm in Bhutan to refugee camps in Nepal, Tsering later said she was surprised with the amount of food rationed:

Tsering: I used to hate the migration part from Bhutan to Nepal, it was very hard, it was really worrisome and sad. I felt a drastic change in we started eating because we were given - other people were giving us food to eat, and there was a difference. We were used to the lush food, healthier environment that we were in. We felt like at the beginning, we were used to having lots of rice, and lots of food because we were farming. When we came to Nepal we were living in camps, so what we were given were bags of rice, and we would look at it and go "Oh my gosh, is that enough for our family?" Because it's very little compared to what we used to have [...] We got used to it and in time, it was enough for all of us.

This narrative is important when comparing their situation in Ottawa. Tsering and other Bhutanese people do not work on farms and grow their own food, so they are not as nearly self-sufficient and independent as they once were. Managing food practices can be read in this light, as a way to handle one's affairs independently. Now in Canada, they have control of the foods they eat, compared to their life in the refugee camps where food was distributed to them.

Norbu mentioned many of the same foods as Tsering but went into more detail about the various food ingredients in the family's regular diet. What I found particularly intriguing with her interview was that she would always note the importance of balancing meals based on nutrition, as well as catering to each family member's taste:

Norbu: So we eat somewhat of a balanced diet. So we'll have daal and we'll have pickle and chutney. Two different things: one is fermented and put into a jar and kept for years and another one is chutney, which we make out of fresh tomatoes. 
So something green, like spinach or bok choy ${ }^{16}$. And sometimes we would have eggs with it [...] egg is almost like a replacement for meat and saag. So we make a lot of things at the same time, so there's options for people like him [grandson] who don't eat meat or green vegetables, so we make something like eggs for him separately. And she [mother] doesn't like eggs, so she will have saag [...] So we make multiple things, so family members can choose. But definitely the staple is rice and lentils.

She also noted particular foods made for special occasions:

Norbu: Sometimes I make $\operatorname{momos}^{17}$, only on holidays.

Food can also be useful in treating a sick family member:

Norbu: Sometimes we would make $k h i c h d i^{18}$, which is like rice, daal, beans - all of that. We call it the 'sick man's food', because it's sick people that eat it.

Radha sees food and cooking as having both nutritional as well as positive effects on one's wellbeing, according to Radha: "And it has to do with your thoughts, your thoughts and words". I understood this statement to mean that health and wellbeing are not merely in the body but can be cultivated. Like Norbu, Radha balanced out her diet, and mixed Nepalese and local ingredients:

Radha: I'm very particular about food habits. If I eat a lot, for instance, I try to cut down the next day, and try to balance it out [...] And I fast two days a week. It's not because of the religious thing but I've been doing that for quite a long time and that helps me a lot. Friday, I try to not eat anything but on Tuesday, a bit of vegetables since it's the middle of the week, no meat, no egg.

Radha also mentioned the importance of changing one's food habits, and for instance,

brings in more traditional food items during festive seasons:

Radha: For instance, if I'm only focusing on the green vegetables, salad, all my children won't learn anything of my culture, and so they need to know, because even if they don't know how to cook, they'll see Mom has been cooking this and Mom has been feeding us this.

${ }^{16}$ Chinese cabbage

${ }^{17}$ Nepali dumplings

${ }^{18}$ Comfort food 
This last sentence, as Radha seemed to imply that cooking is a cultural transmission process. In other words, through cooking foods, she is able to teach her children a bit about her culture, just from them seeing her cooking or eating the food. Food became a source of cultural knowledge. Much like Norbu, Radha was also concerned with the food habits of her family, even trying to get her daughter, a dieter, to eat more food:

Radha: But as I said, when I see new things about food, about nutrition, it really encourages me to find out more about that. And I really like to apply in our life, too. For instance, right now, my daughter is so much into losing weight and whenever she says - we always discuss about that - and I say, "Maybe she's overdoing it?" And I won't say anything, "Don't do this, don't do that" but I'll find a way to make delicious food at home so she'll be tempted, so she won't be overdoing it.

Much like her last point, food was a tool to convey culture. She also used food as a language of its own, to get her message about dieting across to her daughter.

Later in my interview with Tsering, cooking was linked with health maintenance:

Tsering: [...] So if somebody gets sick, then the whole community comes to the house and we pray together for the betterment of the community members. Sometimes the community members come in and start cooking in your kitchen. We do it together, that's our culture.

Tsering wove together notions of cooking, health, sociality, and religion in her interview, demonstrating how these are all interrelated, as well as highlighting the role of the community in health maintenance.

In my final interview, Leeza saw food as a health practice that was very important:

Asli: So in your daily life, do you think cooking could be a part of maintaining our health? And I know you mentioned the community kitchens as something you've done.

Leeza: Yeah, I think cooking is a huge part of who we [South Asians] are, our foods are a huge part of our culture, of our identity. It's based on that we have a 
lot of festivals where we eat a lot of fried of foods and rice. So in order to maintain healthier lifestyles, it's switching up how we cook our foods. On a regular basis, we eat fried chicken and we fry it in a lot of oil back home. Whereas here, you might as well sauté the vegetables instead of frying them. You can cook chicken in a different way: curry in basically broth rather than frying it in a lot of oil, and switching clarified butter (ghee) using low-fat content oils containing omega-3s, instead of lard or butter. Just things like that.

Leeza's narrative suggested that to deny the large role cooking plays in South Asian culture on the whole would be incorrect. In terms of food's role in health, she advised people to change the way food is prepared, not necessarily the food choices themselves.

In summary, food serves a similar purpose across participants: a way of maintaining cultural ties to one's country of origin, through its sights and smells. For Tsering and Norbu. I saw evidence of food also related to wellbeing when considering its links to migration. These Bhutanese food items, like saag, rice, daal and achar are chosen in their daily diets for a sense of nutritional wellbeing because of their health benefits, as well as a sense of emotional wellbeing, a reminder of meals eaten back in Bhutan and Nepal. However, it is important to also consider that food is linked to a sense of agency, as Tsering noted that in the refugee camps, this agency seemed to be lost in these stories, as food was rationed to them, instead of being grown on their farm. A sliver of agency seems to be evident, as participants are now able to purchase and prepare their own traditional meals here in Canada. This agency may also be a read as part of the emotional wellbeing associated with food and cooking as a health practice.

The contrast between these two senses of wellbeing is very apparent with Sangye, who mentioned her enjoyment of drinking alcohol back in Bhutan, as she felt it kept her healthy. For her, foods and drinks from back home serve an emotional purpose. I would argue this trade off between these two senses of food wellbeing is also apparent in 
Leeza's interview. Much like her discussion about the lack of attention to wellbeing in favour of health, her interview seemed to imply that foods, mainly because of their cultural familiarity, are assumed to be nutritious. She uses examples of the many sweets and fried foods in South Asian cuisine. Through changing food preparation practices, this emotional component can still be maintained without having to give up these beloved foods. In these interviews, ideas of health and wellbeing shifted depending on contexts. In some cases, cooking was seen as a health practice, with food items chosen for their health benefits. For others, it was more of a cultural process, used to maintain food identities in Canada, as well as to pass on to the next generation. In other words, health practices are not static, they serve multiple and multi-layered purposes which connect health beliefs to everyday places, making health part of daily life.

\section{Cleanliness}

A minor theme under food which appeared in a few of the interviews was the importance of cleanliness. Abha explained to me what cleanliness meant:
Abha: The concept of cleanliness for the food is that you have to cover it and they have to keep it in the fridge. So things like that is what she's saying about cleanliness for the food, so that it's fresh, it's clean. It's a very hard thing to explain because back in Nepal or somewhere that if you put the food outside, then it would be dirt and dust that would be around the food. But here, it's not like that, it's very different. But they still try to keep it clean as they can, even if it's cleaner here. So they cover the food, and there are insects everywhere so you have to cover your food and take care of it.

The concept of cleanliness is interesting to look at when discussing the role of health and food. To cover one's food makes it healthy because it protects it from dirt and dust. As well, cleanliness can also be a dynamic of one's sense of health as related to wellbeing. Here, a person is able to have a sense of agency or control, so managing food can be an 
example of this. Abha's point that although many of these measures may not be needed here in Canada, she noted that this is an ingrained practice among many Bhutanese people.

Tsering's idea of cleanliness seemed most straightforward:

Tsering: I take showers, so I keep myself clean. I wash my hair, sometimes I put oil in my hair for the health of my hair. I don't wear the same clothes after showers.

In contrast, Leeza sees cleanliness as related more to religion:

Asli: So do you think there's a strong relationship between religion and health, and religion/spirituality?

Leeza: I would say between religion and health...well, religion requires cleanliness, which is also a part of the health and the wellbeing of an individual. There's certain days in a month where people fast or only eat fruits so that when I think of it, I see it as detoxifying days that were meant out in a religious custom or traditions that allows your body to rejuvenate.

For Leeza, the idea of cleanliness seemed to be not so much of a transnational habitus but a leftover religious practice in what she sees as a post-religious world. Much like with food practices, ideas of cleanliness are far from static, and changes depending on context.

\section{Working towards health: employment and income and its effects on health and wellbeing}

One of the major themes in the interviews was the role of employment and income, and its toll on a person's sense of wellbeing. This theme, as mentioned in the review of the literature captures both emotional and socio-economic dimensions of wellbeing.

Radha's discussion began with linking work and wellbeing. She noted the 
importance of paying attention not so much to the tasks of a job but the work

environment:

Asli: Do you think work and employment has an effect on health and wellbeing:

Radha: [...] you can talk to anybody and nobody's happy with their job, whether it's a doctor, an engineer, garment worker - anybody, they have some kind of issues. And if you look at that way, then yes, it's depressing, it is frustrating but if you say, "You know what, it is just a job, it pays my bills that is all". I think if you don't take it too seriously then you will be fine. But keep in mind when you have a job, there is also many things involved, right? Environments, workenvironments and other things as well. How you interact with and getting along with the managers and so many things.

Further along, Radha ended the interview with a conversation between herself and Abha about the lack of conversation concerning work in the Nepali community:

Abha: In the Nepali community, nobody talks about their work. This is very interesting [...] Yes, it is very interesting that we are so much into each other but we talk about our personal issues. Our personal issues are talked about, and usually people give you information or suggestions but we don't really talk about work.

Radha: We don't really talk about work. I mean, with health, we just in general, as I said, but we don't go into detail, right? I mean, I've known somebody for years and years but really, we don't know her. I know her but I don't know her. and maybe the same thing with that person.

Abha: Do you think it's more urban life though? Because I don't know if this is just me but I see that in a very educated, city-life sort of thing.

Radha: I don't know, I think it depends on individuals.

Abha: Yeah, individuals.

From the conversation between Abha and Radha, conversations about work and employment were said to be seldom discussed in the Nepali community, and both seemed to be unsure of why this is the case. This is an interesting contrast to the Bhutanese community, as noted by other informants, who did share their concerns surrounding employment, as well as income: 
Norbu: We're like children because basically whatever money the government is giving, we have to think about what bills to pay...it all gets spent living your life. So there's no other expenses that we have to stretch out that money. Of course, we would have some kind of tension so that community support would be hard.

Recall in the section of this chapter discussing the self-sufficiency of living on her family's farm in Bhutan. Norbu's current socio-economic situation in Canada wipes this income independence away, as her family is dependent on this monthly stipend, which is stretched to accommodate their needs. Later in the interview, Tsering, mentioned some of the issues Norbu has faced since they moved to Canada:

Tsering: She (Norbu) had an occupation back in Nepal, walking around on her own and she had some jobs that she did. She was working in a job, she was busy, she had money, she was self-reliant, self-dependent. And that is one thing that is probably eating her: she is not as independent as she used to be.

Having a job seemed to signal one's independence. Recall earlier in the sub-section detailing food and health that Tsering mentioned that her family grew their own food on their farm. However, some if not all were refugees before coming to Canada, and they are supported financially, which seems to compromise this sense of self-reliance. Fadiman's (1997) ethnographic work with Hmong refugees living in California in the 1980s also found this theme of self-reliance compromised, and one Hmong man stated: "I used to be a real man like any other man, but not now any longer...We only live day by day, just like the baby birds who are only staying in the nest opening their mouths and waiting for their mother bird to bring the worms" (p. 202). Being able to find meaningful work may be beneficial to building a positive sense of wellbeing.

Similarly, Tenzin, an elderly Bhutanese man, expressed this view of work:

Asli: How do you see health? 
Tenzin: We used to work a lot when we were in Bhutan, we used to work on our arms, we used to do a lot of hard work. We also feel working a lot contributed to health. There's no education but we used to work a lot. We used to look after goats, we used to go into the forests, and go around and work and take cattle into the forests.

The role of the farm reappears here, a seemingly significant place for one's mental health, very rich in imagery. Thinking back to days in Bhutan, Tenzin's sense of health is linked directly to days of hard work, with farm labour. The image of the farm for Tenzin and Tsering can be seen as an example of a place associated with health and wellbeing.

Sangye mentions work in Bhutan and Nepal and the change since living in Canada:

Sangye: When I was in a different country (Bhutan and Nepal), it was really hard, especially since we came from Nepal, it was hard. The foods and vegetables were expensive, even though we were eating well. We had to do some hard labour, we were working hard. Overall, life was hard. When we came to Canada, there's no such hard labour we have to do, so we're walking, we're eating healthy. I'm feeling so much better than I used to.

Sangye's opinion of work is interesting because unlike other older Bhutanese participants, like Tsering or Tenzin, she did not look back on work in Nepal and Bhutanese as fondly. Instead, she recalled difficult labour. I understood her response as an example of how health and wellbeing can be differentiated: Sangye now feels healthy because she is no longer doing this hard labour, and taking care of herself.

Work, for many of the older participants is linked to a sense of wellbeing, almost as a part of their identity. Tenzin and Tsering spoke very fondly on their memories of working on their farms, and growing food for themselves. Here, the connection between wellbeing and work became evident, as some participants mentioned having misgivings about receiving government assistance. The role of government assistance appeared to be almost parallel to the role of food rations in Nepal in the camps. In both instances, a sense 
of independence, self-reliance and freedom became compromised.

However, not all reminisce about days of hard labour. Sangye mentions feeling fortunate that in Canada she does not have to do so much hard work, and is able to relax and take care of themselves. Since coming to Canada, they have noticed an improvement in their health. I understood this distinction in perceptions of work for both sets of participants as a way to also differentiate between health and wellbeing. For Tsering and Tenzin, this nostalgia related to farm work in Bhutan was more related to reflecting on a sense of wellbeing to past times of familiarity. While for Sangye, when thinking about work presently in Canada, reflected enjoying free time to care for her health. By examining work, much like wellbeing, health geographers should take into account both socio-economic, as well as emotional understandings of work among newcomers. In this section, interviews illustrated a strong connection between perceptions of work and wellbeing, with many participants linking sense of worth to employment.

One of the most important insights from this chapter was the links between language fluency, socio-economic status, health and a positive sense of wellbeing. Inability to speak fluently in English can prevent one from accessing health services, and without a car, this situation can lead to poorer health as services cannot be utilized. In terms of wellbeing, for some, the inability to earn one's own income instead of relying on government assistance affected their self-esteem negatively. In other words, there is a clear intersection between all of these factors when considering the employment situation for a recent refugee group, like the Bhutanese. 


\section{The role of the neighbouring community in health maintenance}

The last major theme in the interviews was the role(s) the broader community

played in providing health advice and support. This theme is particularly important

because it demonstrated the importance of examining therapeutic networks, the resources

and most importantly, people that participants relied on. In this section, I also tried to pay

attention to the possible untherapeutic networks of health and wellbeing for participants.

Norbu first shared some of her therapeutic networks in her interview:

Abha to Asli: So I asked how much she would rely on her neighbours and people, like if you're sick, then you would go to a neighbour who has similar problems and talk to them about your problems?

Norbu: If I were to get sick, or was something to happen to me, I would definitely ask the community members if they would have suggestions to tell me what I should be doing. If they have experience, they'll tell you what they've gone through, and they'll tell you what hospital to go to, what kind of doctors to see. So yeah, they would discuss with the community members. I don't know what else to say (laughs).

For Norbu, discussing health is not a private matter in the neighbouring Bhutanese community but a public one, which can help others who may later face the same issues or ailments.

Tsering and Sangye echo many of the points addressed by Norbu:

Tsering: Yeah, of course, we have to ask people who have similar issues if they've gone through the same thing. Where do you get medicine? Who's getting sick? Who has issues? We don't hide everything, we talk to each other. So if we go to the next door neighbour, you ask if she has any issues, ask them, "What do you think?" And ask their opinion on anything if they have faced similar situations. You ask how to deal with it, like things like pharmacies.

Sangye: Of course, we need to have community amongst us and get along with them, and we do ask for suggestions from our community members because we don't have the English language, there's somebody in the community who knows. Of course, help them and look at their paper sometimes and help them dealing with their lives here. 
I noticed both women's mention of using community members to aid with health navigation, as well as bills or legal papers showed that the neighbouring community serves a major role in one's navigation of health and social services.

Other evidence of this community support, and its positive effects on health and wellbeing was demonstrated through rituals, as described by Tsering:

Tsering: We do share because that is our culture. So sometimes if somebody is sick, then everybody tries to help, and if you have to take them to the hospital, then usually everybody tries to go, so that you're not by yourself. So if somebody is sick - when we were in Nepal, we used to have the same culture, every day we used to go everywhere together and people used to help each other. And once we came here, our religion has changed, and the way we lead our lifestyle has changed.

In this example, even the home of another member of the Bhutanese community can act as a therapeutic place because other community members are there for emotional support.

The health practice in this case can be read through prayer, as well as community cooking in a patient's home. Communities, as therapeutic networks, can transform ordinary spaces into healthy places in times of need (Palmer, 2012).

It is also important to take into account adjustments in one's therapeutic networks in the diaspora. For Tsering, attending ESL classes appeared to be a major source of positive health effects:

Asli: So the last [question] is: are there particular places that promote health or that you feel emotionally more 'healthier'?

Tsering: I like school because the teachers make me laugh. Teachers make me walk and run. When I say 'hi' (yawn) to the teacher, the teacher makes me do exercises. So sometimes when I don't understand them, I get bored and want to sleep and the teacher will make me exercise, so that keeps me healthy. Maybe the teachers are trying to take care of me because I'm older now, so maybe they're trying to keep me happy because they do different things for me and different exercises. 
In this case, learning the language was a fun exercise. The school was a positive healthy place for Tsering, and she seemed very happy to share these thoughts with me. Later in the interview, Tsering mentioned how religion is a source of change in the diaspora, through her conversion from Hinduism to Christianity. One of the motivations surrounding conversion, according to the literature, is that Bhutanese people in refugee camps convert to Christianity as a way to create a sense of community cohesion, to expand their social networks, and possibly, as a way to fit into Western societies (Duke University, 2013). For Tsering, this conversion ultimately changed her networks:

Tsering: The biggest difference is the rituals and how you used to pray and how you used to do things for the religion's sake, so that's the main difference [...] Following Hinduism, there were Hindu people coming to the house, doing different things in different religions. Now we're here and we believe in Christianity, so I feel like Christian people come to the house and they do different sorts of things together.

Religious conversion is an important event to take into account when considering migrant health and wellbeing because a religious group can provide migrants with new social networks to draw on. Some of the participants mentioned that attending church every Sunday and spending time with church friends were important events in their lives. When discussing health networks, there needs to also be a consideration of those networks created and established in the diaspora.

\section{Untherapeutic networks of health and wellbeing?}

I noticed some untherapeutic or unpleasant instances mentioned by some informants in the interviews. Some were more specific examples, and others were more general comments about adjusting to life in Canada. In my interview with Radha, she and 
Abha went into a discussion amongst themselves about changes in the Nepali community since arriving in Canada:

Radha: [...] Sometimes I feel with myself, "Oh my gosh, what if I don't fit in?" I should try a little bit to stay away from them. I mean, that is unfortunate. I would say because we are here, we are so far away from our country, we don't have anybody. And I have to be honest with you, we are family, we should be living as a family but it's so suffocating. Like we don't - but as I said, we don't talk about work, we don't want to talk about anything.

Abha: We do get together.

Radha: We do get together but you know, it's just like getting together but do we really know each other? No. Do we really make time to understand each other? No. Because we don't make an effort.

Abha: Do you think it was really different in Nepal? Like when we were in Nepal, were we talking to each other?

Radha: In Nepal, the situation is different, Abha, because the thing is we don't need. We have such a big family and we are so occupied with our family, so friends are like second family. Over here, we don't have family, so friends are different. So we easily get hurt over here because, you know, they are our direct contact person. Somehow, we forgot, so we think, "Oh, they're ours. They belong to us". But they don't. So that has to do with sometimes with what we call vulnerability. We become so fragile.

Abha: So basically, they are personal.

Radha: Yes, very personal.

Concerns about being able to connect with other members of one's own community were also mentioned by Tsering:

Tsering: Culture is very different, sometimes when you're a Nepali speaker, sometimes you would just go to somebody and knock on somebody's door and go to somebody's house, even if you don't know that person. They don't like to get to know people here. If they don't know you, they would never knock on your door and say, 'Hi, I'd like to get to know you!' Or, 'I'm coming here!'. Even if you speak the same language, people are not very - they're very work-oriented. They only come for work and leave. They're not very -

Asli: Welcoming? 


\begin{abstract}
Abha: She's not trying to say 'welcoming' exactly but it's like 'warmth'. She's saying lack of warmth.

Tsering: When you get to know somebody, people will say 'Hi' or 'Hello', then they just talk to you nicely outside but if you invite them home then they don't feel as comfortable coming to your house. They don't come to your house and eat your food and getting comfortable in your environment, which is very different because in our culture, it's very normal to go to somebody's house and be comfortable in someone's environment and invite them over and be comfortable in our environment. We sometimes sleep in each other's houses, which doesn't happen here.
\end{abstract}

Abha: So she's wondering if they don't think nicely of us, since we're into that kind of culture. And I'm telling her it doesn't matter.

In both these conversations, both Radha and Tsering noticed that cultural values, such as hospitality and warmth seem to have changed since living in Canada. Because many are focused on their immediate family and careers, cohesion in Nepalese and Bhutanese communities, is not very evident. This idea should, however, be understood in a case by case basis, as inter-group disintegration is not true for all Bhutanese and Nepalese people. For example, Abha who is Nepalese works closely with the Bhutanese community, so she is seen as affiliated with this particular group, and I noticed this affiliation in her mentioning of $u s$.

Other untherapeutic instances are not limited to post-migration life in Canada. Several informants noted unpleasant situations while in Nepal. One example of a possible untherapeutic network was mentioned by Tenzin. He told me a story about his conversation from Hinduism to Christianity prior to migrating:

Tenzin: Before the Hindu religion used to say that in three people, one person obviously dies sick. So when they would be sick, they would pray. And I changed my religion and I feel so much better.

In this case, Tenzin likely felt he was not given positive support regarding his health. Yeshey mentioned something similar, and shared a story about her conversion: 
Yeshey: One of my sons and daughter in-law had some sort of sick spirit and had a brain tumor when the baby was born. She couldn't feed the baby breast milk and when she was going to an operation, did a surgery, from here to here (pointing to the front and back of her head). And then once that was done, she basically had a lot of support from the community from the religion come to the house and pray for my daughter in-law as well. After the whole process was done, after she felt better, I actually started feeling like this community, this society, this religion, actually helped my family get better. There's also no shamans, no herbalists to take care of you, so you have to turn to religion to get support as well. I feel really good after coming here. So my grandchild is five years old, and everything is great in the family.

Similar to Tenzin, Yeshey seemed to see that ideas of health posed through Hinduism provide an untherapeutic setting to talk about illnesses. She felt that conversion and support from Christian groups in Nepal provided her with a better sense of wellbeing and comfort, not only for herself but her family. This is not to say that one religion is better than another but just to show how perceptions of health seem to play a role here in determining one's sense of therapeutic and untherapeutic networks of health. Yeshey's last point about acculturation is also important, because she believed that adapting to the Canadian landscape though religion could make the settlement experience easier.

Besides intra-cultural relations in Canada between Bhutanese and Nepalese people, it is also worth looking at how untherapeutic networks can be linked mainstream Canadian society. Much like with other sections of this chapter, language appeared to play a role in this isolation, according to Tsering:

Tsering: When I moved from Bhutan to Nepal, it took me a long time to adjust there [...] So at least there was language so we could go to different places on my own, go see the villages, go see the areas. But now that I'm here, it's like I have to learn a whole new language and it's hard to move around from one place to another. So it's very different. Anywhere I go, I can't speak. There's no way of asking what the price is, so you don't even go and ask, "How much is this?" So there's no interaction between people. 
This interaction with people is important for newcomers, as a way to make links with others outside of their community, as well as being able to navigate everyday geographies and situations. In reference to the previous section on employment and income independence, connections to other communities may be useful in finding and securing employment for some of the informants.

Similarly, language barriers also had an untherapeutic effect when considering access to health services:

Tsering: The biggest problem I have faced here is if I'm sick, there is a family doctor. I can't just really walk into those family doctors, it's different here. I have to call, set up an appointment, when will they give appointments? I am sick now but they're not looking at me. I have to wait for them to tell me when I should come but by the time that comes, I am already having a hard time [...] The doctor gives you a prescription, you have to go all the way to the pharmacy, sometimes it's not enough money or they say something we don't understand, or sometimes they say your insurance doesn't cover it. We have to come back and ask somebody, then go back again. It's hard. It's a hassle [...] by the time you get the drugs in your hands, you're already OK, already healed. I like the walk-in clinics because they give you the medicine quick. I've heard that you wouldn't die unless death comes for you. I wonder if I will die just looking for medicine.

For Tsering, the hassle just to make a doctor's appointment and get prescriptions proves to be untherapeutic in several ways. First, the costs of prescription drugs not fully covered under her health insurance card, and can be related to the previous theme of income insecurity. Second, the structure of the Canadian health system, with long waits to see physicians lead many to use walk-in clinics instead. Third, the constant back and forth, and walking to these different locations for health care appears to be physically draining, as Tsering is an older woman, this amount of walking can be tiring. Finally, the whole experience in general can be an emotionally draining one as well. Tsering ended this story by mentioning that although she believes death has its own time for each 
person, just the experience of receiving adequate health care seems to be an omen of death. In other words, finding care is a very time-consuming task.

Overall, in this last section, I wanted to illustrate the different roles the neighbouring community play in the lives of people that I spoke to. For the most part, community members play a very large role in health literacy, providing fellow Bhutanese people with health advice and access to health services. In addition, community members appeared to also serve a significant emotional role serving as confidants. Other significant networks of health and wellbeing were present with the role of fellow churchgoers. Even with these idyllic situations, I found evidence that at times, intracommunity communication and cohesion was not always present, and some participants saw this as evidence of acculturation to Canadian society, a turn from older social norms, such as inviting one to sit and even sleep over in their homes. Another possible source of untherapeutic networks some participants mentioned was the role of Hinduism earlier in their lives, in which they felt did not compliment their views of health. These participants felt a conversion to Christianity provided a sense of positive reinforcement and support when a family member was severely ill. Researchers considering the roles social supports systems play in the health of migrants should examine both the possible therapeutic and untherapeutic roles these networks may play.

\section{Conclusions}

The results from these interviews and focus groups have shown that heath indeed connects to several facets of one's life, such as cooking, language and education and employment. In this section, I also aimed to highlight, some of the routines that were performed to maintain one's health, and where these were taking place. Through first 
going into a discussion about definitions of health and wellbeing, these terms at times overlapped or contrasted. For many, health was seen as one's own individual health. For others, wellbeing was seen as an expansive sense of health that included one's immediate family, and sometimes the neighbouring community. These terms clearly shift depending on their contexts.

After noting definitions of both health and wellbeing, I turned to highlighting some of the specific practices associated with health. Walking was seen as a health practice that kept participants content, connected, and active. For Tsering, it was a way to keep her aging body moving, and keep her connected with neighbours. It was also a practice related to wellbeing, as she felt the most comfortable when walking with her husband. For Radha, walking kept her active, as well as emotionally connected to nature. However, walking also highlighted some socio-economic barriers. For Tsering and Norbu who did not have access to a car, walking was an important source of transportation. With this in mind, it is important for us as health researchers to not be too blinded by the emotional benefits of walking that may hide economic underpinnings.

Food preparation and cooking was also noted as a significant health practice. For many, choosing traditional foods items, like rice, greens, lentils and pickles were preferred over a Canadian diet. Also noted in this section was the difference between food that is nutritionally healthy and foods that are emotionally satisfying. Foods were typically seen as 'healthy' if they were traditional, even those with questionable fat content. These unhealthy foods, then, were emotionally satisfying, as they brought out memories of food practices prior to migration. Cooking for many was understood as something related to health but also a practice of cultural transmission. For participants 
like Tsering, Norbu and Radha, preparing traditional meals meant keeping Nepalese and Bhutanese foodways intact. Lastly, much like with walking, cooking also had socioeconomic links. For Bhutanese participants like Tsering, being able to purchase and prepare her own food was a symbol of agency, even if supported through government assistance. This agency was seen as a contrast the food rations given to them while in the refugee camps in Nepal.

Looking at ideas of employment among migrants shows how well-being can be understood emotionally and economically. For many Bhutanese participants, not being able to access employment, due to language barriers, became a source of disappointment and questioning of character. Informants like Tenzin noted this best, as he juxtaposed working in Bhutan on a farm and not being able to do so here. Tsering noted that Norbu likely felt upset with not working in Canada, as she once ran a shop while in Nepal. Others, like Sangye, were thankful for not working in Canada, as she is now able to rest her aging body and pay more attention to her health. Tsering noted that for herself, becoming educated then gainfully employed is good but has the possibility of breaking up her family, as she worried those who become educated will leave the family. Here, income, independence, self-esteem and wellbeing are all very much connected.

Lastly, attention to intra-community relations can also reveal ideas of health and wellbeing. To better highlight these links, I drew upon the concept of therapeutic networks to show how health networks are not necessarily reliant on physical structures but intimate relations. In the first half of this section, I explored positive therapeutic networks, particularly the Bhutanese community, which included intra-community relations Informants like Tsering, Norbu and Sangye mentioned that they received help 
their community members, whether in the form of reading documents or providing health advice. It is also important to take into account that people do make new therapeutic networks after they migrate to a new country. From the interviews, some examples included attending ESL school for Tsering and Norbu, and Radha's mention of work colleagues.

The second half of this section was dedicated to examining possible untherapeutic networks that informants have found in their daily lives. This theme seemed to be evident among both Nepalese and Bhutanese informants. Radha and Abha, both Nepalese women, noted a lack of intimate community cohesion in the Nepali community in Ottawa. They felt that it was not necessarily that their people did not converse or routinely get together but that they did not share personal aspects of their lives. Radha hypothesizes that was do with understandings of 'family' that differed here in Canada compared to Nepal. In Nepal, people's social networks seemed to almost to be limited to one's family, due to the larger size of families, and a smaller number of friends. Radha felt that this notion of family should have been carried over to Canada, where people should begin to see close friends as if they were kin. Tsering felt much the same about the Bhutanese community at times, and noticed that although people may be friendly and greet you on the streets, they are uncomfortable when you insist they come to your home. She fondly remembered times in both Bhutan and Nepal were people would feel at home in your house, as if it was their own. She hypothesized that people are much too focused on work to continue to uphold these cultural values in the diaspora. Another source of untherapeutic experiences were related to instances of religious conversion among Bhutanese participants. Yeshey and Tenzin felt that Hinduism or more specifically, their 
surrounding Hindu community, was not supportive in terms of health. They both felt and noticed greater support from the Christian community, which motivated them to convert to Christianity while in the camps. This section is important to reflect on because it demonstrates links between health and religion, as Tenzin felt Hinduism had more of a fatalistic and bleak outlook on health, while Christianity to him, gave a more positive outlook. In all, this chapter was meant to demonstrate the presence of health to many different parts of one's life, like employment, migration, daily food habits, community networks and others. Wellbeing should also not be ignored in these interconnections. The emotional component of health was also very present in the interviews In this sense, when considering how health and wellbeing can be read geographically, we need to pay attention to how these are enacted through everyday routines, like cooking or walking, for instance, for their layered and nuanced meanings to be evident. 


\section{Chapter 5: Conclusions}

This research began as a way to bridge my interests in immigrant and refugee health, through my background in medical anthropology, and to bring this into conversation with an emerging health geography. The goals of this research were to contribute to this growing body of geographic literature, while also drawing attention to how health and wellbeing could be read through daily routines. In this final chapter, I will note how I have met my research objectives and aims mentioned in the first chapter, through a summary of each of the chapters. From here, I will outline some of the limitations this project faced. I will then turn to some final thoughts of reflection that I call post-reflections of my research journey, while also providing some suggestions for future graduate student researchers about dealing with 'research failure', through another discussion of reflexivity. Finally, I highlight some recommendations for future health geographers considering research with migrant groups, and some areas of development in this stream of inquiry.

\section{A geography of health and wellbeing: overview of research}

How are health and wellbeing understood and defined by Bhutanese and Nepalese people? How can these concepts be understood through everyday life and routines? These were just a few of the questions asked and answered through research conducted with Bhutanese and Nepalese people in Ottawa. One of the most important things to remember when researching these groups is to distinguish between them based on immigration status upon arrival.

The Bhutanese came to Ottawa as government assisted refugees, while a majority 
of Nepalese people came as skilled immigrants. Because of these different immigrant statuses, their respective pre- and post-migration experiences regarding access to health services, employment, and other important aspects of their lives also differ. Through this, one of the aims of my research was to examine how health and wellbeing were understood by participants. To do this, I situated this research in the growing sub-field of health geography, with greater attention to how people experience health, a turning away from a strictly top-down approach to health, focused on measuring health. As well, through the lens of postcolonial theory, these narratives from interviews were meant to be seen as health knowledges, the lived experiences of health and wellbeing of the participants. Below, I provide a synopsis of each of the chapters of the research.

\section{The 'place' of health, wellbeing and therapeutic networks in the literature}

Better understanding health and wellbeing were the main objectives of this research. As such, I felt it appropriate to also review the literature to examine how the main concepts of my research, health, wellbeing, and therapeutic networks were defined. One of the main findings was that health geographers have begun to move away from defining health, and turning their attention to wellbeing. This is likely due to health unfortunately still being associated with disease (Kearns and Collins, 2010). Health was also seen to be a step away from notions of biomedicine for health geographers. On the other hand, wellbeing was at times seen as a subset of a broader notion of health. At the same time, wellbeing was seen to have a long relationship with the discipline of geography. Beginning in economic and welfare geographies, wellbeing was once understood as measurable. More recent developments have pulled the concept into health, emotional and care geographies, with wellbeing positioned in a qualitative light, 
something that is felt and experienced (Atkinson et al., 2012; Flueret and Atkinson, 2007). In terms of works interested in defining wellbeing, Sointu $(2005,2006)$, HealeyOgden and Austin (2011), as well as Doran (2014), were useful in defining how wellbeing could be seen as distinct from neighbouring concepts, like health and wellness. Because the results from the interviews seemed revealed both streams of wellbeing, I referred to both these bodies of literature in my rendering of wellbeing. Here, I cited Dean and Wilson (2009) and Simich et al. (2010), who noted the economic, as well as emotional dimensions of wellbeing for newcomers. Lastly, due to how wellbeing was also linked to culture in the interviews, I added a third dimension related to cultural notions of wellbeing to my review, citing literature from Panelli and Tipa (2007), with attention Māori notions wellbeing in relation to their culture, as well as to processes of colonialism. As well, I noted Elliot and Gillie (1998) who linked wellbeing to experiences of migration and settlement among Indo-Fijian women in Vancouver.

To highlight the importance of the everyday in informing ideas of health, wellbeing and place, as noted, I used the concept of therapeutic networks (Smyth, 2005). Therapeutic networks, as a leading concept allowed me to also examine the untherapeutic places and situations participants found themselves in, as this has been little discussed in the literature. Because this term has not been widely used, I cited works that appeared to have a network-like approach. This concept was useful for two reasons: the focus on the therapeutic networks of immigrants and refugees, very relevant to this research, and the attention to how migration and settlement can shape these networks. For example, health geographer Chakrabarti (2010) used the term to look at how Bengali women in New York City rely on a variety of outlets for advice on pregnancy. As well, medical 
anthropologists, like Thomas (2010) and Krause (2010) used similar concepts, such as transnational networks of care and transnational therapy networks, to examine how these therapeutic networks can also be stretched across scales for Ghanaian and Zimbabwean immigrants in the UK. Others, like Escandell and Tapias (2010) highlighted the emotional dimension of these networks for Bolivian migrants in Spain, to show how emotional geographies may also be relevant when examining therapeutic networks of health and wellbeing.

As a way to tie the literature to the conceptual frameworks discussed above, one of my objectives was to use a relational approach to health. Coined by Cummins et al. (2007), a relational approach in this research involved considering how people may be influenced by a changing sense of both place and health over the life course (p. 1828), and the experience of migration is relevant in shaping these connections. As well, this relational approach recognized and informed my concern with how health and wellbeing can be best understood at the scale of the everyday. Attention to how individuals define health and place can make a major difference in how policy is formulated to meet the needs of people in society. As well, this network-like approach to health and place can reveal how different outlets can be used to inform people's practices and conceptions of health, instead of viewing health practices as measurable behaviours (Macintyre et al., 2002).

Attention to ideas of health and wellbeing came into medical geography in the early 1990s. Prior to this, most research in this sub-field was concerned with quantitative studies of disease ecologies. These medical geographers were interested in how space, or location, could be used as a lens to map these ecologies. This shift to a 'new' medical 
geography (also called health geography) was a turn away from quantitative studies to qualitative works investigating how health could be understood through peoples' conceptions of place. In this research, place typically referred to sites where people lived and experienced life. Here, the term experience was also used as a way to move away from the term perception used by humanist geographers, to account for how these relationships to places can change over time. One of the major theoretical developments to come out of these debates was Gesler's (1992) idea of therapeutic landscapes, which refers to how renowned landscapes can affect people's senses of health. However, in my research, I was more interested in how people, through their daily practices or routines, could create places they associate with health and wellbeing (Dunkley, 2009; Ingold, 1993; Guarnizo, 1997). Therefore, I applied Smyth's (2005) concept of therapeutic networks, the networks or resources, typically outside of the health care system people utilize to maintain their health, everyday.

Literature from disability studies and care geographies were also useful in highlighting the importance of the everyday, as well notions of health and wellbeing. From disability studies in geography, the everyday was unpacked. In much of these works, disability is highlighted in how people manage disability through routines, and this literature illustrated the importance of the body in geography. Care geographies, like health geography, grew out of top-down approaches to care, which resulted in a movement away from care seen as an official responsibility of the state to how care is practiced informally in day-to-day lives of ordinary individuals and communities. This body of literature has two aspects: attention to care as a practice, and caring, the emotional component of care. The former refers to how care is performed, and can 
produce places. Works by Conradson $(2003,2005)$ were paramount here, and he linked care, health, place, and practice through his concept of the self-landscape encounter, which highlights the multi-layered and embodied interactions between people and places. Literature related to caring highlighted how the act of caring can be highly emotional, with both positive and negative experiences for both the caregiver and the cared for (Milligan, 2005). Evans and Thomas (2009) demonstrated this bind in caring with their attention to the care geographies of HIV/AIDS, noting untherapeutic instances, as well as how culture shapes caring practices (Evans, 2010). The last body of literature informing this research was work related to everyday geographies of immigrant and refugee health. Here, I drew attention to how migration, as well as settlement are important lived experiences that can inform how health and wellbeing are defined. Research by Dyck was important here, bringing light to the everyday as a scale where health and wellbeing are negotiated. In her work with South Asian women in Vancouver (2006), Dyck found that practices, like cooking, caring for children, and work, played a role in how these women managed their and their family's health. With medical anthropologist Dossa (2007), much the same was found but both also highlighted the role of religion and prayer influencing conceptions of health. These findings are also close to those found in allied disciplines, like anthropology, with Dennis and Warin's (2009) work with Persian women in Australia, and oral history, with Palmer's (2012) research with Ethiopian refugees in the UK. Overall, in my research, the theoretical use of relationality and therapeutic networks, coupled with the broader literature, revealed how health, wellbeing, and place are very much interconnected. 


\section{Methodological frameworks}

This research was informed by feminist methodologies, which draw attention to the multiplicity and 'situatedness' of knowledges in research (Rose, 1997), as well as a critique of the role of the researcher as objective, unfeeling and all-knowing. In this chapter, I pulled apart some well-known concepts, such as positionality, power, subjectivity, and emotion in qualitative research, and showed my understandings of these in the context of my own research. Feminist methodologies also encourage the use of qualitative methods, like interviewing, as a way to collect data that highlight personal experience and voice. In my research, I used semi-structured interviews to investigate how health and wellbeing were differently defined and understood by eight participants in their daily life, with the duration of the interviews ranging from twenty minutes to an hour. The questions for these interviews covered the definitions of the two terms, followed by how they manifest themselves in daily routines, and finally, to broader questions about the role of one's neighbouring community in providing health advice. An important point to consider was the use of an interpreter to translate interviews between myself and Bhutanese respondents (Shklarov, 2007). Here, I highlighted some of the ethical issues to consider, as well as possible power dynamics that could arise. Another point to consider was the 'placing' of interviews (Elmwood and Martin, 2000; Sin, 2003). All of the interviews with Bhutanese participants took place in a home, while the remaining two took place in a community health centre, and a coffee shop. I felt that allowing participants to choose the location for an interview created interview places, where these narratives could be comfortably shared. Through this chapter, I unpacked the methods of data collection. 


\section{A review of the results}

When analyzing the interview data, I found responses to correspond to five themes: i) definitions of health and wellbeing; ii) walking as a health practice; iii) role of food and cooking to health and wellbeing; iv) links between work and wellbeing; and finally, v) the roles of the neighbouring community for health advice and support. When defining health, I found responses varied: some participants saw health as being an individual task, and other participants viewed health more collectively, linked to broader responsibilities to one's family, with a concern that some family members may become acculturated, and then pursue their own interests. Moreover, wellbeing was also be nuanced in nature, with some participants connecting it to one's emotions, and to broader notions of maintaining the wellbeing or the welfare of one's family and community at large. One participant noted that discussions of wellbeing were shadowed with those surrounding health, and defined wellbeing as the way one leads their life. Wellbeing, then, was something that could achieved, through exercise or healthy living, while health appears to be innate, linked to one's health history and genetic dispositions connected to race.

Examining these definitions of health and wellbeing through practices can reveal how they are connected to everyday life. Walking was one routine or 'step' to maintaining one's health and wellbeing, and in the interviews, it was a multi-layered activity. As an alternative to taking transit or driving, there was evidence of the weight of one's socioeconomic status on one's decision to walk (Bostock, 2001). To some, walking was a form of solace, a way to keep one's body active, despite pain; used to relax one's emotions; as well as a means keep in touch with neighbours, friends and nature, all points evident in 
the literature (Doughty, 2013; Gatrell, 2013). One participant specifically stated that she preferred to walk with her husband, rather than alone. For several others, issues speaking English was seen as a barrier from walking to many places, as literacy was related to enjoying public places. This finding prompted me to link discussions of health literacy (Simich, 2009) to health geography's interest in walking. I felt there needed to be greater attention to how health literacy can affect other areas of one's life outside of accessing health care services. This expansive view of health literacy may also prompt new broader definitions of health in turn, and Simich (2009, p. 10) suggested focusing on popular locations, like ethnic associations, school, and places of worships as key places prompting these discussions.

From walking, discussions turned to the ways in which health and wellbeing could be 'consumed', a focus on food and cooking. Culture played a role here, as the foods associated with the Nepalese and Bhutanese diet were those said to be more healthy, even those with high fat content, like ghee. I took these ideas and divided them into two components of wellbeing related to food: emotional wellbeing, which was linked to memories or meals or certain food items eaten back in Nepal and Bhutan, and nutritional wellbeing, those foods chosen for their health benefits. Outside of food links to health, interviews demonstrated that cooking was a practice used to transmit cultural knowledge, teaching family members, especially children, about cultural food items. A sub-theme also found was linked to the idea of cleanliness. Cleanliness was said to be the practice of covering food from dirt or dust, a practice carried over to Canada. I connected cleanliness, as well as cooking to Guarnizo's (1997) notion of transnational habitus, those practices which become unconsciously maintained through migration. 
Health and wellbeing were also said to be 'worked' on, and this fourth theme revolved around linking work and wellbeing. Many participants discussed the misgivings about receiving government assistance and the difficulties finding work, and for many, having work was linked to one's positive sense of wellbeing and self-esteem. This finding also illustrated a relationship between one's socio-economic status and wellbeing, as shown in the literature (Conradson, 2012). However, this was not the case for all, as some Bhutanese participants appreciated the opportunity to focus on their own health, as work back in Bhutan was labour intensive and tiring. Like the themes of walking and cooking, notions of work varied.

The last finding was concerned with the neighbouring community in informing and influencing notions of wellbeing and health. This finding also provided an opportunity to apply the concept of therapeutic networks, to highlight the various avenues in which people received support. A majority of participants felt that the Bhutanese community played a large role in providing health advice and support during times of need. Support came in the form of translators, health remedies and general health advice, all highlighting how important everyday places and the social interactions within them can inform and maintain the greater health, wellbeing, cohesiveness of a community (Palmer, 2012). These discussions also pointed to particular places, like language school, were also important to maintain health, both through learning, social engagement and exercise. In addition, this theme provided a space to discuss possible untherapeutic networks related to health and wellbeing. Broadly, both Nepalese and Bhutanese participants expressed some discomfort in their communities, as some cultural mores appeared to have changed since arriving to Canada. For example, one Bhutanese woman 
found that there was some warmth missing from some people she has spoken to, and a Nepalese woman noticed that community members do not appear to connect with each other. Another untherapeutic network that was evident was the difficulty in accessing health care, with its many channels, like making appointments, going to walk-in clinics, picking up prescriptions, in addition to language barriers. The last finding discussed was religious conversion, with a couple of participants sharing their motivations in converting to Christianity. In all, through therapeutic networks, both therapeutic and untherapeutic networks and places can be highlighted.

\section{Limitations of the research}

Like any research project, there were limitations to be found. Since this research was heavily reliant on the use of participants' narratives of health and wellbeing, much may have been lost, due to language barriers. In other words, I cannot say for sure that I captured the full extent and meaning behind these narratives. In addition, because of the participants' own schedules and the presence of others during my interviews with Bhutanese participants, it is possible that some of their experiences were not shared. As well, being a researcher and a stranger, I may have not earned complete trust of my participants, and some of them may have felt uncomfortable sharing stories with me.

The sample size for this study was relatively small, mainly due to difficulties with recruitment. It would be incorrect to assume that the experiences and realities for the Nepalese and Bhutanese people I spoke to are comparable and true for all. It is therefore, important to consider the findings of this study contextually. Interviewing various individuals from these groups may have provided very different data, especially 
considering that the Bhutanese participants I spoke to lived in the same neighbourhood. Broadly, migration as a lived experience differs person-to-person, and these differences cannot be overlooked, just because people are of the same national group and or live in same geographic area.

In hindsight, I wished I had more carefully pulled out 'health' from 'wellbeing' in my interview questions. Much like in my review of the literature, there appears to be little differentiation between the two terms, and there were some interviews which participants could not distinguish between the two. In the future, asking Abha beforehand the Nepalese terms for health and wellbeing would be useful. Another major limitation I later found was my initial focus on women. Because I had assumed that only women would be willing to speak to me, I had ignored the possibility of male informants. I had assumed that the Nepalese and Bhutanese, like with my community, would not have women and men close together. In my first interview with Tsering, I found out right away that this was not always the case, as her husband was interested in my interview topics. In the future, it might be best to leave participation open for men and women. This type of scenario, unfortunately, is not covered in literature discussing the use of feminist methodologies while interviewing male participants, and those I came across, instead, noted unpleasant situations (Arendell, 1997; Lee, 1997). Overall, for future studies considering health, wellbeing and place for Bhutanese and Nepalese migrants, it would be imperative to examine these understandings of the terms from a diverse group of participants to obtain a larger picture of the health and wellbeing of these communities.

As a second portion of my limitations, I want to address some of the observations two major gatekeepers to this project, Siffan and Abha, noted in brief email 
questionnaires. Both women were intrigued by the project, seeing it as a good way to empower and voice the concerns of the Bhutanese. However, both noted that its objectives may not have been clear, and that language barriers would be a major obstacle. In terms of recruitment, both did not anticipate long delays. For the future, Siffan suggested that recruitment posters with images, as well as giving presentations about the project may have been better ways to garner more attention. Siffan and Abha recommended that it would have been best to target an existing group, like church or temple groups, as well as trying to contact community leaders to get others to participate. Using email as a way to contact possible participants was also seen as a route that could prevent recruitment delays, as these can be easily distributed through one's networks. A major reflection Abha shared in her responses was the concept of time. Because of other commitments, participating in the research was not seen as a priority to one's family. Abha mentioned that during the time of recruitment, a member of the Bhutanese community fell ill, so others in the community gathered to provide support. As well, many felt more comfortable being interviewed in the presence of their fellow community members, which would impact their willingness to participate. In all, suggesting the use of more visual recruitment posters, email invitations to participate, and the consideration of time and community, Abha and Siffan provided an interesting list of possible solutions to the limitations of this research.

\section{Some ponderings: post-reflections of the research}

Positionality, like reflexivity, is said to be a process always under construction in the research journey, however, very few researchers highlight how they shift in the midst 
of their own work. Here, I want to turn my attention to the change in my positionality through the course of the research, noting particular conversations. In the methodology chapter, I noted my application of reflexivity in Chapter 3, as highly influenced by the work of Pillow (2003), who exposed many different sides to reflexivity in qualitative research. In this section, I unpack the last layer of my personal palimpsest of reflexivity, which I name post-reflection. This post-reflection section includes discussions I have had with other colleagues, as well as non-academics concerning my research. The aim here, is to expose how reflexivity and positionality are constantly under construction 'on' and 'off' the field.

\section{Grad office reflexivity}

One of the best places to discuss research is in the graduate student office in the department. The office that I am located in is a good location for its mix of human and physical geographers, and blend of cohorts. It is a prime location to discuss any issues you may have in the progress of your research, or the place to ask a colleague to give suggestions and recommendations on how to structure presentations and research chapters. One of my colleagues suggested that we meet up to discuss our own progress and go over our understandings of reflexivity, as both of us at the time were beginning to write up our methodology chapters. Our first discussion was very helpful to me because she provided many useful avenues to take this particular chapter. Specifically, I am indebted to her for sending me Pillow's (2003) article on reflexivity, as this provided me a way to better structure my own sense of reflexivity on paper.

Another important article she directed me to was Kohl and McCutcheon's (2014) article, titled 'Kitchen table reflexivity', which helped me ground ideas of reflexivity into 
everyday encounters. The authors state that kitchen table reflexivity "provides a platform to consider, among other things, what it means to occupy positions of power, how these positions change over time, and how relationships with research participants impacts perceptions of positionality in research" (p. 2). They go on to discuss the importance of unpacking positionality, noting that "by employing kitchen table reflexivity, we argue for an in-depth engagement with positionality through formal and informal conversations with other researchers, which develops a richer and more nuanced engagement with positionality that in turn enriches the research processes" (p. 6). I use kitchen table reflexivity as a way to highlight the importance of everyday discussions and conversations to illustrate the journey of graduate student research. As a suggestion, forging these connections and providing spaces for this open dialogue can be one way to curb research burnout, as well as moments of disillusionment. Through these office conversations, I was able to better situate and understand my own research, mainly through the eyes of others.

Another instance of this kitchen table reflexivity was presenting my research to first year Masters students, who were in the process of writing their thesis proposals. Initially embarrassed about sharing my findings about my research, this informal talk became quite a therapeutic experience, as many others in my cohort understood my research issues and sympathized. Several of these first year students thanked me for sharing my experiences, as it helped them think more deeply about possible moments of 'failure' once the time came to conducting their own research. I encountered the same type of reaction when presenting a summary of my research at the Canadian Association of Geographers (CAG) meeting at Simon Fraser University, as well as in my 
department's student research conference. From what I assumed to be the emotional honesty and humour I used in my experience with bouts of research failure, I won the 'People's Choice Award for Best Oral Presentation' in my department. In sum, I see these conversations as an expansion of the 'kitchen table' in which these discussions of reflexivity took place, giving others a better picture of my research, and giving me a clearer image of myself as a researcher. Another suggestion I could give future graduate students is to not only discuss these 'failures' but to also consider presenting your research, to create greater dialogue with other geographers in the field.

\section{Race and personality: 'inner' positionality}

Kohl and McCutcheon (2014) feel that these discussions of reflexivity cannot ignore race, as their idea of the kitchen table as a place for talk comes from Black feminist literature. For myself, race came through one conversation I had with a former student. This conversation really got me thinking more about my racial identity and demeanor as a researcher. Below, is my entry from this conversation:

June 10th, 2015

I remember meeting someone who gave me an interesting comment on my demeanor. She laughed and said I reminded her of herself a few years back: quiet, modest, unassuming. She said that because of this, no one is going to know about any of my achievements, or my research in general, I'd be easily forgotten. Interestingly, she felt this was also tied being a person of colour doing research: many of us act as if we should be thankful for being recognized or invited to events, when in reality, we deserve it. This made me really think and begin to stop downplaying my achievements. There's a fine line between being proud and being arrogant.

Her comments struck me in a couple of ways. For one, as much as I do, especially in the thesis, position myself as a woman of colour, rarely do I think about these positions in 
relation to being in academia, especially in a discipline that is predominately Caucasian and male. Her words also commented on my shyness around new people and distaste for large groups. She encouraged me to make use of networking, especially in the context of future employment. In this case, seeing myself as a woman of colour being a core part of my positionality in research did not seem to bleed through to my inner positionality and personality (cf. Moser, 2008) as Asli, the quiet individual. This silence regarding race in geographic research has prompted some discussion (Mahtani, 2004, 2006; Kobayashi, 1994b, 2006) that should continue to flourish. I am hoping that in future research I discuss, I look more deeply into how race and personality can affect my positionality as a researcher and academic.

\section{Being/becoming a health geographer}

I had not fully considered my role as a health geographer, until I was told by an acquaintance that it was impossible to study health in a geographical light; I would need a science degree to do so. Bringing 'health' and 'geography' together on paper was also a daunting task. As mentioned in my introduction and literature review, this sub-discipline is still relatively young, so the volume of literature is still small. As such, finding published works closely resembling my own has been a major challenge. To compensate, I also turned to works done by public health and nursing researchers, sociologists, medical anthropologists, and refugee studies researchers to aid in theorizing and conceptualizing this work, making this a product of interdisciplinary meta-conversations in the texts.

Another major obstacle was truly considering the weight of the term health. Here, I draw on the writing of Noxolo's (2009), who found a similar dilemma in postcolonial 
geographic writing, and suggests greater attention to embodiment may be useful. Noxolo informs the reader that "one of the first steps towards a consideration of the embodied politics of postcolonial geographic writing is to consider the specific geographies of postcolonial theory itself" (p. 57). My version of the question would be: what are the specific geographies of 'health' in health geographic research itself? This question is not to ask for a narrative about the role of the medical system and access to health services. Rather, it is a call to present and consider the geographies and power behind the term. One route is through the work of medical anthropologists and their attention to medicalization (see Morgan, 1990). Browner (1999) for example, speaks on the medicalization of medical anthropology, as the sub-discipline may "uncritically accept biomedical illness categories and explanations (pp. 135-136). Diseases like cancer or arthritis may begin to be understood only in these medical terms, and Browner fears that medicalization of the sub-discipline can prevent other knowledges from coming to the fore.

For some health geographers, the concern seems to be much the opposite: that as attention to health and place grow, little attention is being paid to the role of health care services and policy. Kearns and Moon (2002, p. 617) feel that this can be related to geography's understanding of the term 'medicine. They write:

geographers tend to have an outdated vision of medicine. This is as problematic as the stereotypes that many health care professionals hold us as demographers, mapmakers and spatial fetishists. The cutting edge of medicine has itself embraced health. We ignore medicine and medical power/knowledge at our peril, but we need to avoid setting up medicine as some kind of folk devil from which we are 'progressively' distancing ourselves.

Instead of assuming that the health care system and biomedicine more broadly write 
against other health knowledges, research exploring other terrains of the medical system by geographers would be warranted. Andrews and Evans (2008) call for more research exposing a relational relationship between these bodies of health geography work ( $\mathrm{p}$. 763). Again, a relational approach to health and place (Cummins et al., 2007) could be useful in exposing multiple meanings and scales associated with health and wellbeing. My own suggestion would be also, as Noxolo notes, to consider the position of an embodied researcher in these works. From this embodied position, other scales of understanding health can be highlighted to peel back layers of health and wellbeing in geography.

\section{Suggestions for future research in health geography}

Through my own challenges in this research, I feel that future health geographers could benefit from my experiences and suggestions. In this section, I want to divide my suggestions into two: i) a focus on possible methodologies and research methods that can be used by health geographers, especially those interested in research with migrant and newcomer groups, and ii) general directions the sub-field could take in terms of inquiry and focus.

\section{Possible methods for health geography}

As noted in my methodology chapter, I had initially hoped to use several methods, including Body Mapping and PhotoVoice, alongside semi-structured interviews. Though this did not end up happening, I still believe that these methods might be useful in future health geography research. Bringing Body Mapping (Gastaldo et al., 2012) from public health, nursing and art therapy can be especially useful in migrant 
health geographies, as images unlike text, can cross linguistic boundaries (cf. Guillemin, 2004). One point to note is that these body maps may even be able to reveal different 'bodies within bodies', showing how one's perception of their body can be influenced by many different factors, like culture, one's community, and broader societies or institutions (see Scheper-Hughes and Lock, 1987). For example, art therapists Lu and Yuen (2012, p. 195) demonstrate in their work how body maps were able to illustrate the emotional scars of colonialism to the bodies of Aboriginal and Mayan women. One point to note for health geographers hoping to use this method would be to consider the amount of time and planning Body Mapping takes, with its many steps of phases. In sum, the use of body maps may also be a good way to reintegrate discussions of the body in health geography (Dorn and Laws, 1994; Johnson, 2009; Parr, 2000, 2002; Kearns, 1993; Hall, 2000), to better illustrate how the body is a site for lived experiences of both health and wellbeing.

A visual method like PhotoVoice may be able to capture participants' conceptualizations of health and wellbeing outside of interview talk. This connection between health and PhotoVoice has already been made, as the main author of this method, Caroline C. Wang (1999), was interested in examining perceptions of women's health. Since her work, there have been many studies also using PhotoVoice as a way to visualize health experiences. For example, Ortega-Alcázar and Dyck (2011) used PhotoVoice as a means to better reveal relationships between health, wellbeing and culture among migrant groups in London, UK. They felt it is was a method that could also write against narratives of othering on the basis of health, and expose 'other' health knowledges. Likewise, Cooper and Yarbrough (2011) used PhotoVoice to reveal rural Guatemalan women's understandings of health. As well, Guell and Oglivie (2015) 
showed how wellbeing could be understood through everyday commuting. Using PhotoVoice in health geography research may also be used to better expose the everyday lived places that participants associate with health. Since researchers do not have access to all parts of a participant's life, the use of a camera can be seen as a way for the participants to better highlight these hidden micro-geographies of health. One point I would note is the financial costs involved with using PhotoVoice, and because of rapid technological changes, it has become increasingly difficult to find disposable cameras. One suggestion could be the use of Polaroid cameras, which have resurged in popularity in the last few years. Although costly, these cameras can be shared among several participants, and may reduce the cost of developing photos. In all, the application of visual methods in health geography research can possibly illustrate new layers and meanings associated with health and wellbeing.

\section{New inquiries through interdisciplinary discussion}

Since the early 1990s, health geography has made interesting changes in inquiry, with the main being a focus on health, and the movement from space to place (Kearns, 1993; Kearns and Joseph, 1993; Kearns and Moon, 2002). My research, through its cited works, has noted many other directions health geography has taken, like the attention to connections between walking, health and wellbeing (Gatrell, 2011, 2013; Andrews et al., 2012; Doughty, 2013), and the embodied and performed geographies of care and caring (Milligan et al., 2007; Conradson, 2005; Evans and Thomas, 2009; Atkinson et al., 2011). However, as this thesis has stressed on many occasions, there needs to be greater attention given to the health geographies of migrant and racialized peoples to show how health and wellbeing can be affected by these varying subject positions and experiences 
of difference. I feel that through continued interdisciplinary dialogue, perhaps these recommendations could be met.

There is some evidence that these cross-disciplinary links are beginning to blossom. As noted in my review of the literature, the term therapeutic landscapes of the mind had been coined by nursing researcher Gastaldo and her interdisciplinary research team (Gastaldo et al., 2004). Specifically, I feel this concept could be a great way to further research related to migration, health and place. Considering the emotional and mental geographies of migration and settlement could be a way to examine mental health issues in newcomer communities more closely. In addition, examining these landscapes of the mind in these communities could also highlight the role of culture in defining and negotiating people's perceptions of mental health. In many communities, mental health issues, like depression, are highly stigmatized, and if health geographers considered these cultural backdrops, they could highlight the geographies in which mental health perceptions and experiences play out. From my own work, I noticed mental health in the form of nostalgia of some participants were longing for their homes in Bhutan and Nepal, I also noticed the role religion played in these narratives. Instead of relegating religion to a sphere of life limited to one holy day a week, or for religious holidays, it appeared that religion played a role in everyday coping. Works in transcultural psychiatry, medical anthropology and refugee studies have made these links. Geographers, in particular, could help identify, highlight, and bring place-based elements to these discussions, and some notable examples include McMichael (2002) and Shoeb et al. (2007) who found Islam to play a large role in everyday life for Somali and Iraqi refugees. Further exploration of these therapeutic landscapes of the mind could be one way to continue 
health research on newcomer and migrant groups.

Interdisciplinary discussions may also benefit health geography theoretically. One of the major issues in health geography is the little attention the discipline has given to theory. Because of this, works have used a variety of theories that have not been fully developed, explored, or contextualized, "as the quest to read place has varied with researchers drawing on positivist, political-economic and humanist traditions" (Kearns and Moon, 2002, p. 610). Admittedly, my attention to theory also has to do with my own interest in postcolonial theory. In this study, I used postcolonial theory as a means to highlight 'other' knowledges associated with health. Although I did cite some useful works by geographers which were noteworthy and helpful, I still feel that postcolonial theory and health geography may be an interesting fit. I found literature from nursing to be novel in their application of postcolonial theory to studies on health, particularly with immigrant and refugee women. These studies recognized that interactions between newcomers and health care workers or professionals can be wrought with complications and power structures established long before. The health care system, to many, is seen as a neocolonial institution, which favours and circulates biomedical understandings of health and disease, overwriting other ways of knowing (Anderson, 2000, 2004; Kirkham and Anderson, 2002; Racine, 2003; Browne et al., 2005; Mohammed, 2006; O' Mahoney and Trung, 2010).

Specifically, one way postcolonial theory could be applied in health geography research is through Said's (1978) concept of othering, which involves defining groups of people as distinctly different from oneself. An example from nursing and critical public health is Lynam and Cowley's piece (2007) which considers practices of marginalization 
to be a possible determinant of health inequity and inequality for immigrants, Marginalization is defined as "the sense of being overlooked, categorized or misrepresented" (p. 146), and the authors use this to show how migrants at times are forced to shift around markers of difference, such as skin colour, accent, and socioeconomic status. This focus on marginalization and othering is also shown in ViruellFuentes' (2007) work with Mexican immigrants in the United States. Attention to these processes of othering may be also useful for health geographers to examine how it can have consequences on a person's sense of mental health and wellbeing. A report on focus groups conducted by Women's Health in Women's Hands (WHIWH, 2003) illustrated the nuanced ways in which othering and racism had on many racialized female participants' sense of self and mental health. Here, attention to othering in health geography may also be a way to introduce discussions of race in the sub-discipline. Moreover, in the context of formal health care interactions, othering has taken place between health care providers and patients, with stereotypes being thrown both ways. This scenario is illustrated in Johnson et al.'s (2004) piece on othering between South Asian patients and Caucasian doctors. From public health, Grove and Zwi (2006) examined how perceived othering can have negative effects on the health and wellbeing of forced migrants, as some people may not feel comfortable utilizing health services once a situation of othering takes place. What these examples of literature related to othering also demonstrate is the scale to which othering can function, and geographers can bring this scalar lens to illustrate how othering can occur from the level of state institutions to the micro-geographies of everyday talk, scales which can all affect one's health and wellbeing. 


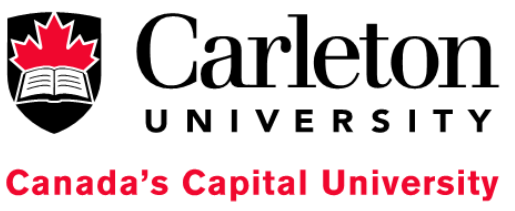

\section{Appendix 1: Letter of Invitation}

Title: A geography of immigrant health and well-being in Ottawa (old title)

Date of ethics clearance: July 21st, 2014

Ethics Clearance for the Collection of Data Expires: May 31st, 2015

October 21, 2014

Dear Madam,

My name is Asli Mahdi and I am a Master's student in the Geography Department at Carleton University. I am writing to you today to invite you to participate in a study on the understandings of health from the perspectives of immigrants in Ottawa. I hope for this study to look at the spaces and practices immigrants feel contribute positively to their physical and emotional health and well-being.

By "spaces", I mean anything from the home, to parks, to neighbourhoods, to community centres, and so on. By "practices", I mean anything from cooking, praying, gardening, walking, use of traditional medicine, conversations with family or community members and many others. Even interactions with your doctor, and brochures that you have read at your doctor's office that you feel has helped shape your understandings of health can be 
considered in this project. Please do not hesitate to give me any ideas or suggestions for this project.

The goal of the project is to look at how health and well-being (emotional satisfaction) can take on many definitions, and for us to move beyond the common idea that health means a body that is free from sickness.

This study will involve one (1) interview of about sixty (60) minutes. This interview with me that will take place in a location of your choice. If you prefer to have interviews done in your home, this is also welcome. With your permission, interviews will be audiorecorded. Once the recording has been typed up and saved on my personal laptop, the audio-recording will be deleted.

While this project does involve some emotional risks, such as questions being too sensitive for you to discuss, care will be taken to protect your identity. I will change your name in all written works and interviews. If you feel uncomfortable with the interviews or the project as a whole, you can let me know and I can try my best to put you into touch with counselling services, if you feel this is what you need.

You will have the right to end your participation in the study at any time, for any reason, up until November 5th, 2014. If you choose to withdraw, all the information you have provided in interviews will be deleted. To withdraw, you may speak to me in person or reach me by email. To promise you that your information will be kept safe, I plan on saving interviews and notes on my personal laptop, which has a password. This research data will only be accessible by the researcher (Asli Mahdi) and the research supervisor (Fran Klodawsky). Names will be changed in all files saved for this research to hide your 
identity.

If you would like to participate in this research project, or have any questions, please contact me at . I am very excited to conduct this project and hope to hear from you. As a token of my appreciation for your participation, I will offer you a small gift as my way of saying thanks. Thank you for your time.

Sincerely,

Asli Mahdi

This project was reviewed by the Carleton University Research Ethics Board, which provided clearance to carry out the research. Should you have questions or concerns related to your involvement in this research, please contact:

\section{REB contact information:}

Professor Andy Adler, Chair

Professor Louise Heslop, Vice-Chair

Research Ethics Board

Carleton University

1325 Dunton Tower

1125 Colonel By Drive

Ottawa, ON K1S 5B6

Tel: 613-520-2517

ethics@carleton.ca 


\section{Appendix 2: Consent Form}

Title: A geography of immigrant health and well-being in Ottawa (old title)

Date of ethics clearance: July 21st, 2014

Ethics Clearance for the Collection of Data Expires: May 31st, 2015

The purpose of this project is look at how health and well-being (emotional satisfaction) is understood by South Asian women in Ottawa. Rather than assuming that to be healthy is to be free of sickness or disease, you will be asked to think of other practices that you feel helps make your feel 'healthy' or happy with your life.

I ,choose to participate in a study

conducted by Asli Mahdi, which looks at understandings of both the terms "health" and "well-being" of immigrants living in Ottawa, and examines at how everyday practices may help define what these two terms mean.

This study involves one (1) interview of sixty (60) minutes With your permission, interviews will be audio-recorded. Once interviews have been typed up, the interview file will be destroyed. This interview of sixty (60) minutes, will involve general questions related to your understandings of health and asks what daily routines you feel contribute to your health. For example, are there any practices, like walking, or food practices that you do everything that you feel contributes to your physical and mental health positively? 
As well, I am interested in examining the locations or spaces in which you feel the most health and happy in your everyday life.

Since this project will ask you about your understandings of the word 'health', you will be promised that if you feel at all uncomfortable with this topic or any of the interview questions, you are able to stop participating in this project, at any time, and for any reason, up until November 5th, 2014. You can withdraw by emailing me at asli.mahdi@carleton.ca. If you withdraw from the study, all information you have provided will be immediately deleted. If you feel you need to speak to someone such as a counsellor or a therapist, I can try my best to make this available to you.

\footnotetext{
All research data, like recorded interview files will be typed up in a Word document, and then saved on my personal laptop. Once this is done, these audio files will be deleted. In all these cases, your identity will still be protected. As well, I will have all the interview notes and files with me at all times. Both of my supervisors will be able to see some of the typed interviews as well as my notes, however, I will change all names to keep your identity private.
}

If you would like a copy of the finished research project, I will be happy to provide this to you. As a token of my appreciation, I will offer you a small for participating. I am looking forward to participating in this project and hearing from you.

Sincerely, Asli Mahdi 
Do you agree to be audio-recorded:

Y Yes

Signature of participant

Signature of researcher
No

Date

Date

This project was reviewed by the Carleton University Research Ethics Board, which provided clearance to carry out the research. Should you have questions or concerns related to your involvement in this research, please contact:

\section{REB contact information:}

Professor Andy Adler, Chair

Professor Louise Heslop, Vice-Chair

Research Ethics Board

Carleton University

1325 Dunton Tower

1125 Colonel By Drive

Ottawa, ON K1S 5B6

Tel: 613-520-2517

ethics@carleton.ca 


\section{Appendix 3: Question sheet for interviews}

Hello, and thank you for agreeing to participate in this project and making time to meet me for this interview. It is greatly appreciated. So what this interview will consist of is twelve (12) questions, and each asks you more specific details about what your feel health is and the things you do that you feel keeps you healthy. I want to remind you that you do not need to tell me any specific details about your health condition, such as having diabetes, or high blood pressure, but to only reveal stories or information that you do not mind sharing.

This interview will be about an hour in length and will be recorded with an audioonly recorder. If you would to stop the interview at any time, please let me know and I will shut off the recorder. If any of my questions are not clear, please let me know and I will try my best to clarify them. If at the end of the interview you have any questions of your own or anything else you would like to share, I would really encourage this. Do you have any questions before we begin?

1. Do you think health is part of our everyday lives?

2. What practices or routines do you do every day that you feel contributes to your health?

3. Are there any things that you do that you feel improves your physical health, like your body or joints? (ex. walking)

4. Are there any practices that do you every day that you feel helps you emotionally, keeping your happy and content for the day?

5. Are there any routines that you do that keep you focused, as well as help your mental health? (for me, I used to write a lot of poetry to let out my frustrations or any other things that pop into mind on paper)

6. In your daily life, do you ever use herbs, spices or any foods your feel are helpful in keeping you healthy? Can you provide any examples? (ex. For me, making a 
pot of chai with ginger, garlic, blackseed oil and honey helps me clear my sore throat).

7. Can we think of cooking as a form of maintaining our health? (Cooking in the sense of the role of food ingredients, and as a social practice)?

8. Do you think your cultural background influences your health practices? I would like to get a sense of how you think being an immigrant or your ethnic background can relate to your health? Do think there is a link between the two?

9. Do you feel that taking care of yourself differs due to gender or age? Do you think that women may have different health practices than men?

10. Do you have family abroad that you reach out to for health advice? Is this a significant source of information for you?

11. Do you speak to anyone in your neighbourhood about your health and for health advice?

12. Do you think that work and employment have an effect on your health and wellbeing? How so?

\section{Questions for coordinators and gatekeepers}

Hello, and thank you agreeing to meet with me once again on the topic $\mathrm{f}$ my project. Although this project has had difficulties concerning recruitment, I feel it is good to still meet and discuss these challenges for future research. I really encourage and appreciate your advice and honesty about your perceptions of my project, its directions as well as limitations. This short interview will consist of seven (7) questions. Do you have any questions before we begin?

1. What were your fist impressions when receiving my email(s) regarding this project?

2. Did you think it was feasible? 
3. What initial issues could you see occurring?

4. Had you any reservations concerning recruitment and its success?

5. Do you have any reasons as to why recruitment for this project was slow? Could there have been changes to its focus, structure, advertisement, methods, etc that could have been changed?

6. What other resources could have been used to advertise this project?

7. Do you have any suggestions for future research with South Asians and any advice that I could use to better promote my research? 


\section{Carleton \\ U N I V E R S I T Y}

Canada's Capital University

\section{Appendix 4: Recruitment Poster}

\section{Participate in a study on immigrant understandings of health and health practices}

To participate in this study, you must be:

$\checkmark$ an immigrant

$\checkmark$ age $30+$

$\checkmark$ be willing to share your own understandings of what health means to who you are as a person.

$\checkmark$ be willing to share what practices you feel contribute to your health - such as going to the doctor and speaking to health workers, cooking, gardening, praying, walking, going to the park, spending time with family, use of traditional medicine, etc. All responses are welcome.

This is a 60 -minute ( 1 hour) interview. You will be asked questions related to your use of the word health and how it relates to your everyday practices and sense of self.

Your identity will be protected, if you wish.

If you agree to participate, you will receive a \$20 Tim Horton's gift card after the interview.

This project has been reviewed and cleared by the Carleton University Research Ethics Board at 613-520-2517 or ethics@carleton.ca. Please contact me, Asli Mahdi, at asli.mahdi@carleton.ca if you are interested in participating or have any questions. 


\section{Bibliography}

Agnew, J. A. (1987). Place and Politics: The Geographical Mediation of State and Society. Boston: Allen \& Unwin.

Anderson, J. (2014). Talking whilst walking: archaeology a geographical of knowledge. Area, 36(3), 254-261.

Anderson, J. M. (2000). Gender, "race", poverty, health and discourses of health reform in the context of globalization: a postcolonial feminist perspective in policy research. Nursing Inquiry, 7(4), 220-229.

. (2004). Lessons from a postcolonial-feminist perspective: suffering and a path to healing. Nursing Inquiry, 11(4), 238-246.

Andrews, G. J., Chen, S., \& Myers, S. (2014). The "taking place" of health and wellbeing: Towards non-representational theory. Social Science \& Medicine, 108, 210-222.

., Hall, E., Evans, B., \& Colls, R. (2012). Moving beyond walkability: on the potential of health geography. Social Science \& Medicine, 75(11), 1925-1932.

Arendell, T. (1997). Reflections on the Researcher-Researched Relationship: A Woman Interviewing Men. Qualitative Sociology, 20(3), 341-368.

Armstrong, P. (2009, October 16). Bhutan refugees struggle to adjust to Canada - The Globe and Mail. Globe and Mail. Vancouver. Retrieved from http://www.theglobeandmail.com/news/british-columbia/bhutan-refugees-struggleto-adjust-to-canada/article4289482/.

Atkinson, S., Fuller, S., \& Painter, J. (Eds.). (2012). Wellbeing and Place. Farnham: Ashgate Publishing, Ltd.

., \& Joyce, K. E. (2010). The place and practices of well-being in local governance. Environment and Planning C: Government \& Policy, 29(1), 133-148.

., Lawson, V., \& Wiles, J. (2011). Care of the body: spaces of practice. Social \& Cultural Geography, 12(6), 563-572.

Barnett, C., Cloke, P., Clarke, N., \& Malpass, A. (2005). Consuming Ethics: Articulating the Subjects and Spaces of Ethical Consumption. Antipode, 37(1), 23-45.

Bennett, K. (2004). Emotionally intelligent research. Area, 36(4), 414-422. 
Besio, K. (2003). Steppin' in it: postcoloniality in northern Pakistan. Area, 35(1), 24-33. . (2005). Telling Stories to Hear Autoethnography: Researching women's lives in northern Pakistan. Gender, Place \& Culture, 12(3), 317-331.

. (2006). Chutes and ladders: Negotiating gender and privilege in a village in Northern Pakistan. ACME: An International E-Journal for Critical Geographers, 5(2), 258-278.

Blunt, A. (2007). Cultural geographies of migration: mobility, transnationality and diaspora. Progress in Human Geography, 31(5), 684-694.

Bondi, L. (2009). Emotional Knowing. In International Encyclopedia of Human Geography (pp. 446-452). Elsevier. Retrieved from http://www.sciencedirect.com/science/article/pii/B9780080449104004259.

. (2014). Understanding feelings: Engaging with unconscious communication and embodied knowledge. Emotion, Space and Society, 10(1), 44-54.

Bostock, L. (2001). Pathways of disadvantage? Walking as a mode of transport among low-income mothers. Health and Social Care in the Community, 9(1), 11-18.

Bourdieu, P. (1990). Structures, habitus, practices. In The Logic of Practice (pp. 52-65). Cambridge: Polity.

Brown, T., McLafferty, S., \& Moon, G. (2009). A Companion to health and medical geography. Malden, MA: Wiley-Blackwell Publishing.

Browne, A. J., Smye, V. L., \& Varcoe, C. (2005). The Relevance of Postcolonial Theoretical Perspectives to Research in Aboriginal Health. Canadian Journal of Nursing, 37(4), 16-37.

Browner, C. H. (1999). On the medicalization of medical anthropology. Medical Anthropology Quarterly, 13(2), 135-40.

Burges Watson, D., Murtagh, M. J., Lally, J. E., Thomson, R. G., \& McPhail, S. (2007). Flexible therapeutic landscapes of labour and the place of pain relief. Health \& Place, 13(4), 865-876.

Canada, Citizenship and Immigration. (2002). Immigration and Refugee Protection Act. Retrieved from http://laws-lois.justice.gc.ca/eng/acts/I-2.5/

refugees. Retrieved from . (2007, May 22). Backgrounder - Bhutanese http://www.cic.gc.ca/english/department/media/backgrounders/2007/2007-0522.asp. 
health care services. Retrieved from

. (2011). Portrait of an Integration Process: accessing http://www.cic.gc.ca/english/resources/research/integration/6-health-care.asp.

. (2015). The refugee system in Canada. Retrieved from http://www.cic.gc.ca/english/refugees/canada.asp.

Canada, Government of. (2013, October 3). Section II - The State and Impact of Mental Illness in the CAF and Canadian Society | Surgeon General's Mental Health Strategy | National Defence | Canadian Armed Forces. Retrieved August 5, 2015, from http://www.forces.gc.ca/en/about-reports-pubs-health/surg-gen-mental-healthstrategy-ch-2.page.

Canada, Multicultual. (n.d.). Encyclopedia of Canada's People/Nepalis: Migration, Arrival, Settlement. Retrieved from http://archive.is/multiculturalcanada.ca.

Canada, Public Health Agency of. (2012, November 25). What is the Population Health Approach? - Population Health. Retrieved from http://www.phac-aspc.gc.ca/phsp/approach-approche/index-eng.php\#def_health.

. (2013, November 25). What Makes Canadians Healthy or Unhealthy? - Population Health Approach. Retrieved from http://www.phacaspc.gc.ca/ph-sp/determinants/determinants-eng.php.

Canadiana. (2005). Canada in the Making - Specific Events \& Topics. Retrieved July 25, 2015, from https://web.archive.org/web/20141120125950/http://www.canadiana.ca/citm/specifi que/immigration_e.html.

Carpiano, R. M. (2009). Come take a walk with me: The "Go-Along" interview as a novel method for studying the implications of place for health and well-being. Health \& Place, 15(1), 263-272.

Carroll, K. (2013). Infertile? The emotional labour of sensitive and feminist research methodologies. Qualitative Research, 15(3), 546-561.

Casey, E. S. (2001). Between Geography and Philosophy: What Does It Mean to Be in The Place-World? Annals of the Association of American Geographers, 91(4), 683693.

Cattell, V., Dines, N., Gesler, W., \& Curtis, S. (2008). Mingling, observing, and lingering: Everyday public spaces and their implications for well-being and social relations. Health \& Place, 14(3), 544-561.

Centre, Somerset West Community Health. (2012). The Ottawa Newcomer Clinic. Retrieved from http://www.swchc.on.ca/ottawa-newcomer-clinic. 
Chakrabarti, R. (2010). Therapeutic networks of pregnancy care: Bengali immigrant women in New York City. Social Science \& Medicine, 71(2), 362-369.

Chakrabarty, D. (2000). Provincializing Europe: Postcolonial Thought and Historical Difference. Princeton, N.J.: Princeton University Press.

Chouinard, V. (2012). Mapping bipolar worlds: lived geographies of "madness" in autobiographical accounts. Health \& Place, 18(2), 144-151.

$70-80$.

. (2000). Disability, Geography and Ethics. Philosophy \& Geography, 3(1),

Conradson, D. (2005). Landscape, care and the relational self: Therapeutic encounters in rural England. Health \& Place, 11(4), 337-348.

. (2012). Wellbeing: Reflections on Engagements. In S. Atkinson, J.

Painter, \& S. Fuller (Eds.), Wellbeing and Place (pp. 15-34). Farnham: Ashgate.

. (2003). Spaces of care in the city: the place of a community drop-in centre. Social \& Cultural Geography, 4(4), 507-525.

Cooper, C. M., \& Yarbrough, S. P. (2010). Tell me--show me: using combined focus group and photovoice methods to gain understanding of health issues in rural Guatemala. Qualitative Health Research, 20(5), 644-653.

Cresswell, T. (2009). Place. In International Encyclopedia of Human Geography (pp. 169-177). Elsevier. Retrieved from http://www.sciencedirect.com/science/article/pii/B9780080449104003102.

Crooks, V. A., \& Chouinard, V. (2006). An embodied geography of disablement: chronically ill women's struggles for enabling places in spaces of health care and daily life. Health \& Place, 12(3), 345-352.

Cummins, S., Curtis, S., Diez-Roux, A. V., \& Macintyre, S. (2007). Understanding and representing "place" in health research: A relational approach. Social Science \& Medicine, 65(9), 1825-1838.

Damianakis, T., \& Woodford, M. R. (2012). Qualitative Research With Small Connected Communities: Generating New Knowledge While Upholding Research Ethics. Qualitative Health Research, 22(5), 708-718.

Dean, J. A., \& Wilson, K. (2008). “I spent nine years looking for a doctor”: exploring access to health care among immigrants in Mississauga, Ontario, Canada. Social Science \& Medicine, 66(6), 1271-83. 
. (2009). "Education? It is irrelevant to my job now. It makes me very depressed ....": exploring the health impacts of under/unemployment among highly skilled recent immigrants in Canada. Ethnicity \& Health, 14(2), 185-204.

. (2010). "My health has improved because I always have everything I need here...": A qualitative exploration of health improvement and decline among immigrants. Social Science \& Medicine, 70(8), 1219-1228.

Dennis, S., \& Warin, M. (2007). Domestic Temporalities: Sensual Patterning in Persian Migratory Landscapes. Indo-Pacific Journal of Phenomenology, 7(2), 1-9.

Dickson-Swift, V., James, E. L., Kippen, S., \& Liamputtong, P. (2009). Researching sensitive topics: qualitative research as emotion work. Qualitative Research, 9(1), $61-79$.

Doran, N. J. (2014). Experiencing Wellness Within Illness: Exploring a MindfulnessBased Approach to Chronic Back Pain. Qualitative Health Research, 24(6), 749760.

Dorn, M., \& Laws, G. (1994). Social Theory, Body Politics, and Medical Geography: Extending Kearns's Invitation. The Professional Geographer, 46(1), 106-110.

Doughty, K. (2013). Walking together: The embodied and mobile production of a therapeutic landscape. Health \& Place, 24, 140-146.

Dowling, R. (2010). Power, Subjectivity, and Ethics in Qualitative Research. In I. Hay (Ed.), Qualitative Research Methods in Human Geography (3rd ed., pp. 26-39). Don Mills, ON: Oxford University Press.

Dunkley, C. M. (2009). A therapeutic taskscape: Theorizing place-making, discipline and care at a camp for troubled youth. Health \& Place, 15(1), 88-96.

Dunn, K. (2010). Interviewing. In I. Hay (Ed.), Qualitative Research Methods in Human Geography (Third, pp. 101-127). Don Mills, ON: Oxford University Press.

Dyck, I. (1989). The Immigrant Client: Issues in Developing Culturally Sensitive Practice. Canadian Journal of Occupational Therapy, 56(5), 248-255.

. (1992). Managing Chronic Illness: An Immigrant Woman's Acquisition and Use of Health Care Knowledge. American Journal of Occupational Therapy, 46(8), 696705.

. (1995a). Putting chronic illness "in place". Women immigrants' accounts of their health care. Geoforum, 26(3), 247-260. 
. (1995b). Hidden Geographies: The Changing Lifewolds of Women with Multiple Sclerosis. Social Science \& Medicine, 40(3), 307-320.

. (1999). Using Qualitative Methods in Medical Geography: Deconstructive Moments in a Subdiscipline? The Professional Geographer, 51(2), 243-253.

. (2003). Feminism and Health Geography: twin tracks or divergent agendas? Gender, Place \& Culture, 10(4), 361-368.

. (2006). Travelling tales and migratory meanings: South Asian migrant women talk of place, health and healing. Social \& Cultural Geography, 7(1), 1-18.

., \& Dossa, P. (2007). Place, health and home: Gender and migration in the constitution of healthy space. Health \& Place, 13(3), 691-701.

., Kontos, P., Angus, J., \& McKeever, P. (2005). The home as a site for long-term care: Meanings and management of bodies and spaces. Health \& Place, 11(2), 173185.

., Lewis, N., \& McLafferty, S. (2001). Geographies of Women's Health. London and New York: Routledge.

., Lynam, J. M., \& Anderson, J. M. (1995). Women talking. Women's Studies International Forum, 18(5-6), 611-626.

Ebrahimi, Z. (2012). Frail Elders' Experiences With and Perceptions of Health. Qualitative Health Research, 22(11), 1513 - 1523.

Edwards, R . (1998). A critical examination of the use of interpreters in the qualitative research process. Journal of Ethnic and Migration Studies, 24(1), 197-208.

. (2013). Power and trust: an academic researcher's perspective on working with interpreters as gatekeepers. International Journal of Social Research Methodology, 16(6), 503-514.

Elliott, S. J., \& Gillie, J. (1998). Moving experiences: a qualitative analysis of health and migration. Health \& Place, 4(4), 327-339.

Ellis, C. (2007). Telling Secrets, Revealing Lives: Relational Ethics in Research With Intimate Others. Qualitative Inquiry, 13(1), 3-29.

Elmwood, S., \& Martin, D. (2000). "Placing" Interviews: Locations and Scales of Power in Qualitative Research. The Professional Geographer, 52(4), 649-657.

England, K. V. L. (1994). Getting Personal: Reflexivity, Positionality, and Feminist Research. The Professional Geographer, 46(1), 80-89. 
. (2006). Producing Feminist Geographies: Theory, Methodologies, and Research Strategies. In S. Aitken \& G. Valentine (Eds.), Approaches to Human Geography (pp. 286-297). London: SAGE Publications.

English, J., Wilson, K., \& Keller-Olaman, S. (2008). Health, healing and recovery: Therapeutic landscapes and the everyday lives of breast cancer survivors. Social Science \& Medicine, 67(1), 68-78.

Escandell, X., \& Tapias, M. (2010). Transnational Lives, Travelling Emotions and Idioms of Distress Among Bolivian Migrants in Spain. Journal of Ethnic and Migration Studies, 36(3), 407-423.

Ettema, D., \& Smajic, I. (2015). Walking, places and wellbeing. The Geographical Journal, 181(2), 102-109.

Evans, J., \& Jones, P. (2011). The walking interview: Methodology, mobility and place. Applied Geography, 31(2), 849-858.

Evans, M. (2012). Feeling my way: emotions and empathy in geographic research with fathers in Valparaíso, Chile. Area, 44(4), 503-509.

Evans, R. (2010). Children's Caring Roles and Responsibilities within the Family in Africa. Geography Compass, 4(10), 1477-1496.

., \& Thomas, F. (2009). Emotional interactions and an ethics of care: Caring relations in families affected by HIV and AIDS. Emotion, Space and Society, 2(2), 111-119.

Fadiman, A. (1997). The Spirit Catches You and You Fall Down: A Hmong Child, Her American Doctors, and the Collision of Two Cultures. New York: Farrar, Straus, and Giroux.

Fanon, F. (1952). Black Skin, White Masks. New York: Grove Press. . (1961). The Wretched of the Earth. New York: Grove Press.

Fleuret, S., \& Atkinson, S. (2007). Wellbeing, health and geography : a critical review and research agenda. The New Zealand Geographer, 63, 106-118.

Gastaldo, D., Andrews, G. J., \& Khanlou, N. (2004). Therapeutic landscapes of the mind: theorizing some intersections between health geography, health promotion and immigration studies. Critical Public Health, 14(2), 157-176. 
., Khanlou, N., Massaquoi, N., \& Curling, D. (2005). Revisiting Personal is Political: Immigrant Women's Health Promotion. Toronto. Retrieved from http://www.migrationhealth.ca/sites/default/files/files/3-Page-ExecutiveSummary.pdf.

., Magalhaes, L., Carrasco, C., \& Davy, C. (2012). Body-Map Storytelling as Research: Methodolopgical considerations for telling the stories of undocumented workers through body mapping. Toronto. Retrieved from http://www.migrationhealth.ca/undocumented-workers-ontario/body-mapping.

Gatrell, A. (2011). Mobilities and Health. Burlington, VT: Ashgate. . (2013). Therapeutic mobilities: Walking and "steps" to wellbeing and health. Health \& Place, 22, 98-106.

Gesler, W. M. (1992). Therapeutic landscapes: medical issues in light of the new cultural geography. Social Science \& Medicine, 34(7), 735-746.

. (1993). Therapeutic landscapes: theory and a case study of Epidauros, Greece. Environment and Planning D: Society and Space, 11(2), 171-189.

$95-105$. . (1996). Lourdes: healing in a place of pilgrimage. Health \& Place, 2(2),

., \& Kearns, R. A. (2002). Culture/place/health. New York: Psychology Press.

Ghorashi, H. (2008). Giving Silence a Chance: The Importance of Life Stories for Research on Refugees. Journal of Refugee Studies, 21(1), 117-132.

Gilmartin, M., \& Berg, L. D. (2007). Locating postcolonialism. Area, 39(1), 120-124.

Gilroy, P. (1993). The Black Atlantic: Modernity and Double Consciousness. Cambridge, MA: Harvard University Press.

Government of Canada, Health Canada, Public Affairs, C. and R. B. (2014, June 2). Health Care System. Retrieved from http://www.hc-sc.gc.ca/hcs-sss/index-eng.php. , Strategic Policy Branch, C. H. A. D. (2010, July 26). Canada Health Act. Retrieved from http://www.hc-sc.gc.ca/hcs-sss/mediassur/cha-lcs/index-eng.php.

Gregory, D., Johnston, R., Pratt, G., Watts, M., \& S. Whatimore (eds.). (2009) Reflexivity In The Dictionary of Human Geography (Fifth, p. 627). London: WileyBlackwell. 
Grove, N. J., \& Zwi, A. B. (2006). Our health and theirs: forced migration, othering, and public health. Social Science \& Medicine, 62(8), 1931-1942.

Guarnizo, L. E. (1997). The Emergence of a Transnational Social Formation and The Mirage of Return Migration Among Dominican Transmigrants. Identities, 4(2), 281-322.

Guell, C., \& Ogilvie, D. (2013). Picturing commuting: photovoice and seeking wellbeing in everyday travel. Qualitative Research, 15(2), 201-218.

Guillemin, M. (2004). Understanding illness: using drawings as a research method. Qualitative Health Research, 14(2), 272-289. ., \& Gillam, L. (2004). Ethics, Reflexivity, and "Ethically Important Moments" in Research. Qualitative Inquiry, 10(2), 261-280.

Hall, E. (2000). "Blood, brain and bones": taking the body seriously in the geography of health and impairment. Area, 32(1), 21-29.

Hall, S. (1994). Cultural Identity and Diaspora. In P. Williams \& L. Chrisman (Eds.), Colonial Discourse and Post-Colonial Theory (pp. 392-403). New York: Columbia University Press.

Hands, Women's Health in Women's. (2003). Racial Discrimination as a Health Riskfor Female Youth: Implications for Policy and Healthcare Delivery in Canada. Toronto. Retrieved from http://accessalliance.ca/wpcontent/uploads/2015/03/ePubRepRacDiscYouth.pdf.

Harvey, D. (2014). Exploring women's experiences of health and well-being in remote northwest Queensland, Australia. Qualitative Health Research, 24(5), 603-614.

Healey-Ogden, M. J., \& Austin, W. J. (2011). Uncovering the lived experience of wellbeing. Qualitative Health Research, 21(1), 85-96.

Ho, E. L.-E., \& Hatfield, M. E. (2011). Migration and everyday matters: Sociality and materiality. Population, Space and Place, 17(6), 707-713.

Hochschild, A. R. (1983). The managed heart: commercialization of human feeling. Berkeley: University of California Press.

Holland, J. (2007). Emotions and Research. International Journal of Social Research Methodology, 10(3), 195-209.

Hopkins, P. E. (2007). Positionalities and knowledge: Negotiating ethics in practice. ACME: An International E-Journal for Critical Geographers, 6(3), 386-394. 
Ian, C., \& Michelle, H. (2003). Cross over food: re-materializing postcolonial geographies. Transactions of the Institute of British Geographers, 28(3), 296-317.

Imrie, R. (2000). Disabling Environments and the Geography of Access Policies and Practices. Disability \& Society, 15(1), 5-24.

. (2000). Disability and discourses of mobility and movement. Environment and Planning A, 32(9), 1641-1656.

. (2004). Disability, embodiment and the meaning of the home. Housing Studies, 19(5), 745-763.

. (1996). Ablest Geographies, Disablist Space: towards a reconstruction of Gollerdge's geography and the geography and the disabled. Transactions of the Institute of British Geographers, 21(2), 397-403.

., \& Edwards, C. (2007). The Geographies of Disability: Reflections on the Development of a Sub-Discipline. Geography Compass, 1(3), 623-640.

., \& Kumar, M. (1998). Focusing on Disability and Access in the Built Environment. Disability \& Society, 13(3), 357-374.

Ingold, T. (1993). The temporality of the landscape. World Archaeology, 25(2), 152-174.

Jazeel, T., \& McFarlane, C. (2010). The limits of responsibility: a postcolonial politics of academic knowledge production. Transactions of the Institute of British Geographers, 35(1), 109-124.

Johnson, J. L., Bottorff, J. L., Annette, J., Grewal, S., Hilton, B. A., \& Clarke, H. (2004). Othering and Being Othered in the Context of Health Care Services. Health Communication, 16(2), 255-271.

Johnston, L. (2009). The Body. In International Encyclopedia of Human Geography. (pp. 326-331). Elsevier. Retrieved from http://www.sciencedirect.com/science/article/pii/B978008044910400924X.

Jones, B., \& Ficklin, L. (2012). To walk in their shoes: Recognising the expression of empathy as a research reality. Emotion, Space and Society, 5(2), 103-112.

Jones, P., Bunce, G., Evans, J., Gibbs, H., \& Hein, J. R. (2008). Exploring Space and Place With Walking Interviews. Journal of Research Practice, 4(2), 1-9.

Karnieli-Miller, O., Strier, R., \& Pessach, L. (2009). Power relations in qualitative research. Qualitative Health Research, 19(2), 279-289. 
Kearns, R. A. (1991). The place of health in the health of place: The case of the Hokianga special medical area. Social Science \& Medicine, 33(4), 519-530.

. (1993). Place and Health: Towards a Reformed Medical Geography. The Professional Geographer, 45(2), 139-147.

. (1996). AIDS and medical geography: embracing the Other? Progress in Human Geography, 20(1), 123 - 131.

. (2014). The health in "life's infinite doings": A response to Andrews et al. Social Science \& Medicine, 115, 147-149.

., \& Collins, D. (2010). Health Geography. In T. Brown, S. McLafferty, \& G. Moon (Eds.), A Companion to Medical and Health Geography (pp. 15-32). Sussex, UK: Wiley-Blackwell Publishing.

., \& Joseph, A. E. (1993). Space in its place: Developing the link in medical geography. Social Science \& Medicine, 37(6), 711-717.

., \& Moon, G. (2002). From medical to health geography: novelty, place and theory after a decade of change. Progress in Human Geography, 26(2), 605625.

Kenan Institute, Duke University. (2013). Introduction to Bhutanese Refugees in Nepal. Retrieved from http://kenan.ethics.duke.edu/uprootedrerouted/introductions/nepal.html.

Kibria, N. (1995). Family Tightrope: The Changing Lives of Vietnamese Americans. Princeton, N.J.: Princeton University Press.

Kickbusch, I. (2001). Health literacy: addressing the health and education divide. Health Promotion International, 16(3), 289-297. . (2002). Health literacy: a search for new categories. Health Promotion International, 17(1), 1-2.

King, R. (2012). Geography and Migration Studies: Retrospect and Prospect. Population, Space and Place, 18(2), 134-153.

Kiptinness, C., \& Dharod, J. M. (2011). Bhutanese Refugees in the United States: Their Dietary Habits and Food Shopping Practices Upon Resettlement. Journal of Hunger \& Environmental Nutrition, 6(1), 75-85.

Kirkham, S. R., \& Anderson, J. M. (2002). Postcolonial Nusring Scholarship: From Epistemology to Method. Advances in Nursing Science, 25(1), 1-17. 
Kitchin, R. (1998). "Out of Place", "Knowing One"s Place': Space, power and the exclusion of disabled people. Disability \& Society, 13(3), 343-356.

. (2000). The Researched Opinions on Research: Disabled people and disability research. Disability \& Society, 15(1), 25-47.

., \& Law, R. (2001). The Socio-spatial Construction of (In)accessible Public Toilets. Urban Studies, 38(2), 287-298.

Kobayashi, A. (1994). Coloring the Field: Gender, "Race," and the Politics of Fieldwork. The Professional Geographer, 46(1), 73-80.

. (2004). GPC Ten Years On: is self-reflexivity enough? Gender, Place \& Culture, 10(4), 345-349. 13(1), 33-38.

. (2006). Why Women of Colour in Geography? Gender, Place \& Culture, ., \& Peake, L. (2007). Unnatural discourse. "Race" and gender in geography. Gender, Place \& Culture, 1(2), 225-243.

Kohl, E., \& McCutcheon, P. (2014). Kitchen table reflexivity: negotiating positionality through everyday talk. Gender, Place \& Culture, 22(6), 747-763.

Krause, K. (2008). Transnational Therapy Networks among Ghanaians in London. Journal of Ethnic and Migration Studies, 34(2), 235-251.

Lawson, V. (2009). Instead of Radical Geography, How About Caring Geography? Antipode, 41(1), 210-213.

Lee, D. (1997). Interviewing men: Vulnerabilities and dilemmas. Women's Studies International Forum, 20(4), 553-564.

Lefebvre, H. (1991). Critique of Everyday Life, Volume 2. New York: Verso.

Liamputtong, P., \& Suwankhong, D. (2015). Therapeutic landscapes and living with breast cancer: the lived experiences of Thai women. Social Science \& Medicine, $128,263-71$.

Lipworth, W. (2011). Balance, Balancing, and Health. Qualitative Health Research, $21(5), 714-725$.

Litva, A., \& Eyles, J. (1995). Coming out: exposing social theory in medical geography. Health \& Place, 1(1), 5-14. 
Lu, L., \& Yuen, F. (2012). Journey women: Art therapy in a decolonizing framework of practice. Arts in Psychotherapy, 39(3), 192-200.

Lynam, M. J., \& Cowley, S. (2007). Understanding marginalization as a social determinant of health. Critical Public Health, 17(2), 137-149.

Macintyre, S., Ellaway, A., \& Cummins, S. (2002). Place effects on health: how can we conceptualise, operationalise and measure them? Social Science \& Medicine, 55(1), $125-139$.

Mackian, S. C. (2009). Well-Being. In International Encyclopedia of Human Geography (pp. 235-240). Elsevier. Retrieved from http://www.sciencedirect.com/science/article/pii/B9780080449104003539.

Madge, C. (1998). Therapeutic landscapes of the Jola, The Gambia, West Africa. Health \& Place, 4(4), 293-311.

Magalhães, L., Gastaldo, D., Martinelli, G., Canada, D., \& Hentges, A. (2009). The Many Faces of Brazilian Immigrants in Ontario. Toronto. Retrieved from http://www.migrationhealth.ca/sites/default/files/BMTC_English_2009.pdf?sid=10.

Mahler, S. J. (1995). American Dreaming: Immigrant Life on the Margins. Princeton, N.J.: Princeton University Press.

Mahtani, M. (2004). Mapping race and gender in the academy: the experiences of women of colour faculty and graduate students in Britain, the US and Canada. Journal of Geography in Higher Education, 28(1), 91-99.

. (2006). Challenging the Ivory Tower: Proposing anti-racist geographies within the academy. Gender, Place \& Culture, 13(1), 21-25.

Mains, S. P., Gilmartin, M., Cullen, D., Mohammad, R., Tolia-Kelly, D. P., Raghuram, P., \& Winders, J. (2013). Postcolonial migrations. Social \& Cultural Geography, 14(2), 131-144.

Manning, L. K. (2013). Navigating hardships in old age: exploring the relationship between spirituality and resilience in later life. Qualitative Health Research, 23(4), $568-575$.

Marte, L. (2011). Afro-Diasporic Seasonings: Food Routes and Dominican Place-Making in New York City. Food, Culture and Society, 14(2), 181-204.

Martin, G. P., Nancarrow, S. A., Parker, H., Phelps, K., \& Regen, E. L. (2005). Place, policy and practitioners: On rehabilitation, independence and the therapeutic landscape in the changing geography of care provision to older people in the UK. Social Science \& Medicine, 61(9), 1893-1904. 
Mayer, J. D., \& Meade, M. S. (1994). A Reformed Medical Geography Reconsidered. The Professional Geographer, 46(1), 103-106.

McDowell, S. (2009). Memory. In International Encyclopedia of Human Geography (pp. 59-63). Elsevier. Retrieved from http://www.sciencedirect.com/science/article/pii/B9780080449104003886

McEwan, C. (2003). Material Geographies and Postcolonialism. Singapore Journal of Tropical Geography, 24(3), 340-355. 00163

., \& Goodman, M. K. (2010). Place Geography and the Ethics of Care: Introductory Remarks on the Geographies of Ethics, Responsibility and Care. Ethics, Place \& Environment, 13(2), 103-112.

McLees, L. (2012). A Postcolonial Approach to Urban Studies: Interviews, Mental Maps, and Photo Voices on the Urban Farms of Dar Es Salaam, Tanzania. The Professional Geographer, 65(2), 283-295.

McMichael, C. (2002). "Everywhere is Allah's Place": Islam and the Everyday Life of Somali Women in Melbourne, Australia. Journal of Refugee Studies, 15(2), 171188.

., \& Manderson, L. (2004). Somali women and well-being: Social networks and social capital among immigrant women in Australia. Human Organization, 63(1), 88-99.

McMullin, J. (2005). The call to life: revitalizing a healthy Hawaiian identity. Social Science \& Medicine, 61(4), 809-820.

Menjívar, C. (2000). Fragmented Ties: Salvadoran Immigrant Networks in America. Berkeley: University of California Press. . (2002). The Ties that Heal: Guatemalan Immigrant Women's Networks and Medical Treatment. Human Development, 36(2), 437-466.

Messias, D. K. H. (2002). Transnational health resources, practices, and perspectives: Brazilian immigrant women's narratives. Journal of Immigrant Health, 4(4), 183200.

Mikkonen, J., \& Raphael, D. (2010). Social Determinants of Health: The Canadian Facts. Toronto. Retrieved from http://www.thecanadianfacts.org/The_Canadian_Facts.pdf.

Milligan, C. (2005). From home to "home": Situating emotions within the caregiving experience. Environment and Planning A, 37(12), 2105-2120. 
., Atkinson, S., Skinner, M., \& Wiles, J. (2007). Geographies of care: A commentary. New Zealand Geographer, 63(2), 135-140.

., Gatrell, A., \& Bingley, A. (2004). "Cultivating health": Therapeutic landscapes and older people in northern England. Social Science \& Medicine, 58(9), $1781-1793$.

Milligan, C., \& Wiles, J. (2010). Landscapes of Care. Progress in Human Geography, 34(6), 736-754.

Mohammed, S. A. (2006). Moving Beyond the "Exotic": Applying Postcolonial Theory in Health Research. Advances in Nursing Science, 29(2), 98-109.

Moon, G. (2009). Health Geography. In International Encyclopedia of Human Geography (pp. 35-45). Elsevier. Retrieved from http://www.sciencedirect.com/science/article/pii/B9780080449104003382

Morgan, L. M. (1990). The medicalization of anthropology: a critical perspective on the critical-clinical debate. Social Science \& Medicine, 30(9), 945-950.

Moser, S. (2008). Personality: A new positionality? Area, 40(3), 383-392.

Moss, P. (1995). Embeddedness in Practice, Numbers in Context: The Politics of Knowing and Doing. The Professional Geographer, 47(4), 442-449.

. (1997). Negotiating spaces in home environments: Older women living with arthritis. Social Science \& Medicine, 45(1), 23-33.

., \& Dyck, I. (1996). Inquiry into environment and body: women, work, and chronic illness. Environment and Planning D: Society and Space, 14(6), 737-753.

., \& Dyck, I. (1999). Body, Corporeal Space, and Legitimating Chronic Illness: Women Diagnosed with M.E. Antipode, 31(4), 372-397.

., \& Dyck, I. (2003). Women, Body, Illness: Space and Identity in the Everyday Lives of Women with Chronic Illness. Lanham: Rowman \& Littlefield Publishers.

Mullings, B. (1999). Insider or outsider, both or neither: Some dilemmas of interviewing in a cross-cultural setting. Geoforum, 30(4), 337-350.

Nash, C. (2002). Cultural geography: postcolonial cultural geographies. Progress in Human Geography, 26(2), 219-230.

Nimmon, L. E. (2007a). Within the eyes of the people: using a photonovel as a consciousness-raising health literacy tool with ESL-speaking immigrant women. Canadian Journal of Public Health, 98(4), 337-40. 
. (2007b). ESL-speaking immigrant women's disillusions: voices of health care in Canada: an ethnodrama. Health Care for Women International, 28(4), 381396.

Noxolo, P. (2009). "My Paper, My Paper": Reflections on the embodied production of postcolonial geographical responsibility in academic writing. Geoforum, 40(1), 5565 .

., Raghuram, P., \& Madge, C. (2012). Unsettling responsibility: Postcolonial interventions. Transactions of the Institute of British Geographers, 37(3), 418-429.

Nutbeam, D. (2008). Health literacy as a public health goal: a challenge for contemporary health education and communication strategies into the 21 st century. Health Promotion International, 15(3), 259-267.

O’Mahoney, J. M., \& Donnelly, T. T. (2010). A Postcolonial Feminist Perspective Inquiry into Immigrant Women's Mental Health Care Experiences. Issues in Mental Health Nursing, 31(7), 440-449.

Omariba, D. W. R., \& Ng, E. (2011, February 25). Immigration, Generation and Selfrated Health in Canada: On the Role of Health Literacy. Canadian Journal of Public Health. Retrieved from http://journal.cpha.ca/index.php/cjph/article/view/2310.

Organization, World Health. (2003). WHO Definition of Health. Retrieved from http://www.who.int/about/definition/en/print.html.

. (2009). Track 2: Health literacy and health behaviour. World Health Organization. Retrieved from http://www.who.int/healthpromotion/conferences/7gchp/track2/en/.

Ortega-Alcazar, I., \& Dyck, I. (2012). Migrant narratives of health and well-being: Challenging "othering" processes through photo-elicitation interviews. Critical Social Policy, 32(1), 106-125.

Palmer, D. (2012). "It's as if you are dropped from the moon": exploring Ethiopian cultural places and well-being through oral histories with Ethiopian forced migrants in London. Oral History, 40(1), 67-78.

Panelli, R., \& Tipa, G. (2007). Placing Well-Being: A Maori Case Study of Cultural and Environmental Specificity. EcoHealth, 4(4), 445-460

. (2009). Beyond foodscapes: considering geographies of Indigenous well-being. Health \& Place, 15(2), 455-65.

Parr, H. (1997a). Mental health, public space, and the city: questions of individual and collective access. Environment and Planning D: Society and Space, 15(4), 435-454. 
(1997b). Naming names: brief thoughts on disability and geography. Area, 29(2), $173-176$.

. (1998a). Mental health, ethnography and the body. Area, 30(1), 28-37.

. (1998b). The politics of methodology in 'post-medical geography': mental health research and the interview. Health \& Place, 4(4), 341-353.

. (1999). Delusional geographies: the experiential worlds of people during madness/illness. Environment and Planning D: Society and Space, 17(6), 673-690.

. (2000). Interpreting the "hidden social geographies" of mental health: ethnographies of inclusion and exclusion in semi-institutional places. Health \& Place, 6(3), 225-237.

. (2002). Medical geography: diagnosing the body in medical and health geography, 1999-2000. Progress in Human Geography, 26(2), 240-251.

. (2003). Medical geography: care and caring. Progress in Human Geography, 27(2), 212-221.

. (2004). Medical geography: critical medical and health geography? Progress in Human Geography, 28(2), 246-257.

., Philo, C., \& Burns, N. (2004). Social geographies of rural mental health: experiencing inclusions and exclusions. Transactions of the Institute of British Geographers, 29(4), 401-419.

Pillow, W. (2003). Confession, catharsis, or cure? Rethinking the uses of reflexivity as methodological power in qualitative research. International Journal of Qualitative Studies in Education, 16(2), 175-196.

Poland, B., \& Pederson, A. (1998). Reading Between the Lines: Interpreting Silences in Qualitative Research. Qualitative Inquiry, 4(2), 293-312.

Pollock, G., Newbold, K. B., Lafreniere, G., \& Edge, S. (2012). Discrimination at the Doctor's Office: Immigrants and Refugee Experiences. Critical Social Work, 13(2). Retrieved from http://www1.uwindsor.ca/criticalsocialwork/discriminationindoctoroffice.

Popke, J. (2009). Geography and ethics: non-representational encounters, collective responsibility and economic difference. Progress in Human Geography, 33(1), 81 90. 
Poureslami, I., Rootman, I., Doyle-Waters, M. M., Nimmon, L., \& Fitzgerald, J. M. (2011). Health literacy, language, and ethnicity-related factors in newcomer asthma patients to Canada: a qualitative study. Journal of Immigrant and Minority Health, 13(2), 315-22.

Racine, L. (2003). Implementing a postcolonial feminist perspective in nursing research related to non-Western populations. Nursing Inquiry, 10(2), 91-102.

Raghuram, P., \& Madge, C. (2006). Towards a method for postcolonial development geography? Possibilities and challenges. Singapore Journal of Tropical Geography, 27(3), 270-288.

Raphael, D. (2009). Social Determinants of Health: Canadian Perspectives (2nd Edition). Canadian Scholars' Press.

Rizal, D. (2004). The unknown refugee crisis: expulsion of the ethnic Lhotsampa from Bhutan. Asian Ethnicity, 5(2), 151-177.

Robinson, J. (2003). Postcolonialising Geography: Tactics and Pitfalls. Singapore Journal of Tropical Geography, 24(3), 273-289.

Rootman, I., \& Ronson, B. (2005). Literacy and health research in Canada: where have we been and where should we go? Canadian Journal of Public Health, 96(2), 562577.

Rose, G. (1997). Situating knowledges: positionality, reflexivities and other tactics. Progress in Human Geography, 21(3), 305-320.

Rosenburg, \& W., M. (1998). Medical or health geography? Populations, peoples and places. Population, Space and Place, 4, 211-226.

Said, E. W. (1978). Orientalism. New York: Vintage Books.

Scheper-Hughes, N., \& Lock, M. M. (1987). The Mindful Body: A Prolegomenon to Future Work in Medical Anthropology. Medical Anthropology Quarterly, 1(1), 641.

Schwanen, T., \& Atkinson, S. (2015). Geographies of wellbeing: an introduction. The Geographical Journal, 181(2), 98-101.

Shklarov, S. (2007). Double vision uncertainty: the bilingual researcher and the ethics of cross-language research. Qualitative Health Research, 17(4), 529-538.

Shoeb, M., Weinstein, H. M., \& Halpern, J. (2007). Living in Religious Time and Space: Iraqi Refugees in Dearborn, Michigan. Journal of Refugee Studies, 20(3), 441-460. 
Sidaway, J. D. (2000). Postcolonial geographies: an exploratory essay. Progress in Human Geography, 24(4), 591-612.

Silk, J. (2000). Caring at a Distance: (Im)partiality, Moral Motivation and the Ethics of Representation - Introduction. Ethics, Place \& Environment, 3(3), 303-309.

Simich, L. (2009). Health literacy and immigrant populations. Public Health Agency of Canada and Metroplis Canada. Toronto. Retrieved from http://canada.metropolis.net/pdfs/health_literacy_policy_brief_jun15_e.pdf.

., Este, D., \& Hamilton, H. (2010). Meanings of home and mental well-being among Sudanese refugees in Canada. Ethnicity \& Health, 15(2), 199-212.

Sin, C. H. (2003). Interviewing in "Place": The socio-spatial construction of interview data. Area, 35(3), 305-312.

Singleton, K., \& Krause, E. M. S. (2009). Understanding Cultural and Linguistic Barriers to Health Literacy. The Online Journal of Issues in Nursing, 14(3). Retrieved from http://www.nursingworld.org/mainmenucategories/anamarketplace/anaperiodicals/oj in/tableofcontents/vol142009/no3sept09/cultural-and-linguistic-barriers-html.

Smith, K. E. (2006). Problematising power relations in "elite" interviews. Geoforum, $37(4), 643-653$.

Smith, S. J. (2005). States, markets and an ethic of care. Political Geography, 24(1), 120.

Smyth, F. (2005). Medical geography: therapeutic places, spaces and networks. Progress in Human Geography, 29(4), 488-495.

Sointu, E. (2005). The rise of an ideal: tracing changing discourses of wellbeing. The Sociological Review, 53(2), 255-274. . (2006). Recognition and the Creation of Wellbeing. Sociology, 40(3), 493-510.

Staeheli, L. A., \& Brown, M. (2003). Where has the welfare gone? Introductory remarks on the geographies of care and welfare. Environment and Planning A, 35(5), 771777.

Takahashi, L. M., \& Magalong, M. G. (2008). Disruptive social capital: (un)healthy socio-spatial interactions among Filipino men living with HIV/AIDS. Health \& Place, 14(2), 182-97.

Temple, B., \& Edwards, R. (2002). Interpreters/Translators and Cross-Language Research: Reflexivity and Border Crossings. International Journal of Qualitative Methods, 1(2), 1-12. 
Thomas, F. (2007). Eliciting emotions in HIV / AIDS research : a diary-based approach. Area, 39(1), 74-82.

. (2010). Transnational health and treatment networks: Meaning, value and place in health seeking amongst southern African migrants in London. Health \& Place, 16(3), 606-612.

Thrift, N. (1999). Steps to an Ecology of Place. In D. Massey, J. Allen, \& P. Sarre (Eds.), Human Geography Today (pp. 295-323). Cambridge: Wiley.

Tolia-Kelly, D. (2004a). Locating processes of identification: studying the precipitates of re-memory through artefacts in the British Asian home. Transactions of the Institute of British Geographers, 29(3), 314-329.

. (2004b). Materializing post-colonial geographies: examining the textural landscapes of migration in the South Asian home. Geoforum, 35(6), 675-688.

. (2004c). Landscape, race and memory: biographical mapping of the routes of British Asian landscape values. Landscape Research, 29(3), 277-292.

Vallianatos, H., \& Raine, K. (2008). Consuming food and constructing identities among Arabic and South Asian immigrant women. Food, Culture and Society, 11(3), 355373.

Viruell-Fuentes, E. A. (2007). Beyond acculturation: immigration, discrimination, and health research among Mexicans in the United States. Social Science \& Medicine, 65(7), 1524-35.

., Miranda, P. Y., \& Abdulrahim, S. (2012). More than culture: Structural racism, intersectionality theory, and immigrant health. Social Science and Medicine, 75(12), 2099-2106.

Wagman, P. (2011). Perceptions of Life Balance Among a Working Population in Sweden. Qualitative Health Research, 21(3), 410 - 418.

Wang, C. C. (1999). Photovoice: A Participatory Action Research Strategy Applied to Women's Health. Journal of Women's Health, 8(2), 185-192.

Watts, J. H. (2008). Emotion, empathy and exit: reflections on doing ethnographic qualitative research on sensitive topics. Medical Sociology Online, 3(2), 3-14. Retrieved from http://www.medicalsociologyonline.org/current/jhwatts.html.

Wendt, D. C., \& Gone, J. P. (2012). Urban-indigenous therapeutic landscapes: A case study of an urban American Indian health organization. Health \& Place, 18(5), 1025-1033. 
Widdowfield, R. (2000). The place of emotions in academic research. Area, 32(2), 199208.

Wiles, J. (2003). Daily geographies of caregivers: Mobility, routine, scale. Social Science \& Medicine, 57(7), 1307-1325.

Williams, A. (2002). Changing geographies of care: Employing the concept of therapeutic landscapes as a framework in examining home space. Social Science \& Medicine, 55(1), 141-154.

., \& Garvin, T. (2004). Taking stock: geographical perspectives on women and health in Canada. The Canadian Geographer, 48(1), 29-34.

Wilson, K. (2003). Therapeutic landscapes and First Nations peoples: An exploration of culture, health and place. Health \& Place, 9(2), 83-93.

Wilton, R. D. (1996). Diminished worlds? The geography of everyday life with HIV/AIDS. Health \& Place, 2(2), 69-83. $51(2), 254-264$.

Winchester, H. P. M. (1996). Ethical issues in interviewing as a research method in human geography. Australian Geographer, 2(1), 117-131.

Woelz-Stirling, N., Manderson, L., Kelaher, M., \& Benedicto, A.-M. (2001). Young Women in Conflict: Filipinas growing up in Australia. Journal of Intercultural Studies, 22(3), 295-306.

Woodby, L. L., Williams, B. R., Wittich, A. R., \& Burgio, K. L. (2011). Expanding the notion of researcher distress: the cumulative effects of coding. Qualitative Health Research, 21(6), 830-838.

Zanchetta, M., \& Poureslami, I. (2006). Health Literacy Within the Reality of Immigrants' Culture and Language. Canadian Journal of Public Health, 97(2), 2630. 